\title{
58th Annual Symposium of the International Society for Clinical Electrophysiology of Vision (ISCEV 2020)
}

\author{
14-16 September 2020
}

(C) Springer-Verlag GmbH Germany, part of Springer Nature 2020

\section{Foreword}

Welcome to the Special Meeting Issue of Documenta Ophthalmologica for the ISCEV 2020 Symposium. Though we are unable to assemble in person this year, the meeting organizers have assembled an informative program of presentations which reflect the depth and breadth of the international community dedicated to clinical electrophysiology of vision. The Abstract Editing Committee has made every effort to optimize the abstracts for clarity and readability-we have made no attempts to address questions of scientific or medical validity. This year, as it is not possible to address follow-up questions to the presenters face-to-face, we have included the email address of the corresponding author with each abstract to facilitate such communication. Enjoy the meeting!

Abstract Editing Committee: Scott E. Brodie, Chair, Mitchell Brigell, Mary Johnson, Jonathan Lyons, Anne Moskowitz, Richard Smith

\section{Oral Session 1: Pediatric electrophysiology}

O1.1 The role of visual electrodiagnostics in clinical evaluation of children with neurofibromatosis type 1

Tekavčič Pompe, Manca ${ }^{1}$, Šuštar, Maja ${ }^{1}$

University Eye Clinic, Ljubljana, Slovenia

Purpose To evaluate visual electrodiagnostic findings in children with neurofibromatosis type 1 (NF1) with or without optic pathway glioma.

Methods 35 children (21 boys and 14 girls, age $4-18$ years) with genetically confirmed NF1 were included. As part of ophthalmic workup, all children had visual electrodiagnostic testing, which included pattern ERG and pattern reversal VEP to full- and half-field stimulation in older children, while in younger children only pattern reversal VEP to full-field monocular stimulation was performed. All children had neuroimaging (brain MRI) where visual pathway gliomas were searched for, however in two of the children MRI was performed after visual electrodiagnostic evaluation.

Results 16/35 (46\%) children have documented optic pathway glioma with MRI. In 9/16 (56\%) children with confirmed glioma, VEP was abnormal and correlated with MRI findings. In 6/19 (32\%) children with documented optic pathway glioma, VEP was within normal limits, while one child couldn't perform reliable visual electrodiagnostic testing. In 19/35 (54\%) children optic pathway glioma was not detected by MRI. Among these children, four $(21 \%)$ had abnormal VEP responses. In five children, neuroimaging was performed after visual electrodiagnostic evaluation had shown abnormal responses. In one child an optic pathway glioma was confirmed.

Conclusion Visual electrodiagnostic tests have an important role in clinical evaluation of children with NF1. In cases with confirmed optic pathway glioma, normal VEP responses might imply intact visual pathway function. In cases where MRI testing was not yet performed, abnormal VEP responses imply the need for further neuroimaging of the visual pathway.

Conflict of interest: None

Funding: None

Award applications: None

Corresponding author email: manca.tekavcic-pompe@ guest.arnes.si

O1.2 The benefits of a modified inferior electrode placement for paediatric pattern visual evoked potentials

Marmoy, Oliver $\mathbf{R}^{1,2}$, Gitsels, Lisanne A.,4, Handley, Sian E. ${ }^{1,3}$, Blackwell, Charlotte ${ }^{1}$, Thompson, Samantha ${ }^{1}$, Cortina-Borja, Mario ${ }^{3,4}$, Thompson, Dorothy A. ${ }^{1,3}$

${ }^{1}$ Tony Kriss Visual Electrophysiology Unit, Clinical and Academic Department of Ophthalmology, Great Ormond 
Street Hospital for Children NHS Trust, London, UK; ${ }^{2}$ Manchester Academy for Healthcare Science Education, Manchester, UK; ${ }^{3}$ UCL-GOS Institute for Child Health, London, UK; ${ }^{4}$ Ulverscoft Vision research Group UCLGOSH Institute of Child Health, London, UK

Purpose To investigate whether an electrode over the inion (Iz) is superior for recording of pattern reversal visual evoked potentials (prVEPs) in children compared to the midoccipital $(\mathrm{Oz})$ position recommended in the ISCEV VEP standard.

Methods Data from 1497 patients (mean age 62.2 months; range 1.6-236 months, IQR: 24.5-87.4) who had prVEPs reported as normal for age were tested at our laboratory between the years 2016-2019 and were included for study. PrVEP stimuli concordant with the ISCEV VEP standard, large and small check widths $\left(50^{\prime}\right.$ and $\left.12.5^{\prime}\right)$, were included in a range of check widths $\left(200^{\prime}-6.25^{\prime}\right)$. Stimuli were presented with a reversal rate of 3 reversals/second to children looking with both eyes open at a $30^{\circ}$ field of $82 \mathrm{~cd} /$ $\mathrm{m}^{2}$ mean luminance. PrVEPs were recorded simultaneously from a midline mid-occipital $(\mathrm{Oz})$ electrode position and a lower, inion (Iz) electrode position, each referred to a midfrontal (Fz) electrode. P100 amplitudes and latencies were measured by two independent observers and verified through systematic sampling by an experienced clinical scientist. Data were analysed as a function of amplitude and latency according to age through logarithmic modelling, limits of agreement, regression analysis and descriptive statistics to ascertain the age-dependence of any differences between electrode sites.

Results We found that the majority of paediatric patients have larger pattern reversal VEPs over Iz than using the ISCEV VEP standard $\mathrm{Oz}$ placement. The significantly higher amplitude prVEPs seen over Iz than $\mathrm{Oz}$ appeared to normalise to an average difference within $10 \%$ of $\mathrm{Oz}$ amplitude at the age of 90 months (7.5 years). Of note, 63 patients $(0.04 \%)$ would have been considered to have abnormally low amplitude prVEPs using $\mathrm{Oz}$ alone, whereas responses were normal using the Iz amplitude, suggesting that this extra electrode site may reduce false positive findings in practice. In addition, we found the differences between prVEPs at $\mathrm{Oz}$ and $\mathrm{Iz}$ were related to check width used. Importantly, we found no consistent or significant difference in peak-time between $\mathrm{Oz}$ and $\mathrm{Iz}$ electrode sites.

Conclusion This large cohort of paediatric patients demonstrate a clinical advantage of using an electrode located over the inion (Iz), in addition to the ISCEV standard $\mathrm{Oz}$ placement, for higher amplitude prVEPs. This is particularly useful for young children under 7.5 years of age. Interestingly, the prVEP amplitude difference between electrode sites appears sensitive to maturation of spatial selectivity.

Conflict of interest: None

Funding: None

Award applications: None

Corresponding author email: o.marmoy@nhs.net
O1.3 VEPs and ERGs in children with CLN2 late infantile neuronal ceroid lipofuscinosis

Handley, Sian E. ${ }^{1,2}$, Marmoy, Oliver R. ${ }^{1,3}$, Gissen, Paul $^{2,4}$, Thompson, Dorothy A. ${ }^{1,2}$

${ }^{1}$ Tony Kriss Visual Electrophysiology Unit, Clinical and Academic Department of Ophthalmology, Great Ormond Street Hospital, London, UK.; ${ }^{2}$ UCL-GOS Institute for Child Health, London, UK; ${ }^{3}$ Manchester Academy for Healthcare Science Education, Manchester, UK; ${ }^{4}$ Metabolic Medicine, Great Ormond Street Hospital, London, UK

Purpose Our purpose is to characterise the visual electrophysiology of children with CLN2 "late infantile" neuronal ceroid lipofuscinosis (LINCL). Children with this neurodegenerative condition present with speech delay and seizures between ages of 2-4 years followed by rapid neurocognitive decline. The onset of outer retinal dystrophy typically by 4 years progresses from the para-fovea to the periphery and leads to blindness by 7-10 years.

Methods As part of treatment trials using the recently approved intracerebroventricular device to deliver enzyme replacement therapy (ERT) across the blood brain barrier, 15 children (seven male) identified with CLN2 disease caused by variants in the TPP1 gene had visual electrophysiology tests at a mean age of 6.8 years (median 6 years, mode 4 years, range 3.9-17.5 years). Flash ERGs (skin electrodes) and VEPs were carried out in 13/15, (two families declined photic stimulation) and pattern reversal (PR) VEPs in 14/15. ERT began an average of 1.9 years before testing (range 0.4-3.1 years) in 10 children, was not planned yet in one (age 6.1 years) and was due to start at the same admission as testing in four (age range 3.9-4.8 years). Retinal features on fundus examination $(n=11)$, OCT $(n=5)$ and/or Optos $(n=7)$ (three had both types of imaging) were graded using the Weill Cornell LINCL Ophthalmic Severity Scale (OSS). This OSS grade was compared to a functional grade of macular pathway function derived from the smallest check width to produce a PRVEP $(n=13)$.

Results ERGs were normal in six (46.15\%) children, mean age (8.1 years), three of whom were untreated and three had been on treatment for a mean of 1.9 years. ERGs indicated cone dysfunction in one $(7.69 \%)$ at age 4.8 years, were absent in five $(38.46 \%)$ mean age 6.78 years and were abandoned in one (7.69\%). Flash VEPs were absent in $2 / 13(15.38 \%)$ aged 3.9 years and 5.9 years (one absent flash ERG, one not tested), and present in 11/13 tested (84.62\%). All 11 patients with flash VEPs had intrusive background EEG and 3/11 showed time locked high amplitude sharp transients. PRVEPs were evident in $13 / 15$ patients: to ISCEV small checks in five (33.33\%), ISCEV large checks in six $(40 \%)$, larger checks (200' or 100') in two (13.33\%), absent in one $(6.67 \%)$ and not possible in one $(6.67 \%)$ due to lack of fixation towards the PR stimulus. The smallest check width to produce a PRVEP closely associated with the Weill Cornell LINCL OSS R ${ }^{2}$ $=0.74$. Of the six with normal ERGs and PRVEPs at first assessment, an attenuated form of CLN2 was diagnosed in three with a LINCL OSS of one (two already on active ERT). 
Conclusion CLN2 retinopathy shows a very broad range of retinal presentation with age in this case series. PRVEP evidence of macular pathway function has a potential to identify windows of therapeutic opportunity for vision preserving treatment. Though more data are required, the smallest check width to produce a PRVEP appears to be closely associated with the LINCL ophthalmic scale and should be considered in assessment of these children.

Conflict of interest: None

Funding: None

Award applications: Eberhard Dodt Memorial Award

Corresponding author email: sian.handley@gosh.nhs.uk

\section{O1.4 Long-term visual outcomes of children born to opioid-dependent, methadone-maintained mothers suggest a foetal opioid syndrome}

Hamilton, Ruth ${ }^{1}$, Butler, Laura ${ }^{2}$, McCulloch, Daphne $\mathbf{L}^{3}$, Mcneil, Aine ${ }^{4}$, Michael, Kaleena ${ }^{5}$, Mulvihill, Alan', Spowart, Katherine ${ }^{7}$, Waterson Wilson, Janice ${ }^{4}$, Mactier, Helen ${ }^{8}$

${ }^{1}$ Department of Clinical Physics \& Bioengineering, Royal Hospital for Children, NHS Greater Glasgow \& Clyde, Glasgow, UK; ${ }^{2}$ Tennant Institute of Ophthalmology, Gartnavel General Hospital, NHS Greater Glasgow \& Clyde, Glasgow, UK; ${ }^{3}$ Department of Optometry, University of Waterloo, Waterloo, Canada; ${ }^{4}$ Orthoptic Service, Department of Ophthalmology, Royal Hospital for Children, NHS Greater Glasgow \& Clyde, Glasgow, UK; ${ }^{5}$ Glasgow Centre for Ophthalmic Research, Gartnavel General Hospital, NHS Greater Glasgow \& Clyde, Glasgow, UK; ${ }^{6}$ Paediatric Ophthalmology, Princess Alexandra Eye Pavilion, NHS Lothian, Edinburgh, UK; ${ }^{7}$ Specialist Childrens Services, Woodside Health Centre, NHS Greater Glasgow \& Clyde, Glasgow, UK; ${ }^{8}$ Princess Royal Maternity, NHS Greater Glasgow \& Clyde, Glasgow, UK

Purpose Abnormal neonatal VEPs, independently associated with methadone exposure, and abnormal visual findings at 6 months were previously reported in a cohort of children born to opioid-dependent, methadone-maintained mothers. We now describe long-term follow-up visual findings in the same cohort at $8-10$ years of age.

Methods Children attended for comprehensive visual assessment; when that could not be facilitated, their case notes were reviewed. Prenatal drug exposure was established from maternal history and urinalysis and infant urine and meconium. Near and distance acuity, stereovision, binocular fusion, strabismus, eye movements and VEPs were assessed by researchers masked to prenatal exposure. A 'fail' was predetermined as any of 1) acuity worse than $0.2 \operatorname{logMAR} ; 2$ ) presence of strabismus; 3 ) presence of nystagmus; 4) poor stereovision.

Results 152 children (102 exposed, 50 comparison) were eligible for inclusion. Long-term data were available for 133 (89 opioid-exposed (OE) and 44 comparison). Of the 133 children, 33 (22 OE, 11 comparison) attended for assessment and 100 (67 OE and 33 comparison) had case notes reviewed. Fifty of the $89(56 \%)$ OE children failed the visual criteria vs 8 of the $44(18 \%)$ comparison children. The $50 \mathrm{OE}$ children who failed had some combination of strabismus (47/50), poor distance acuity (30/50), nystagmus (17/50), poor stereovision $(14 / 50)$ and/or poor near vision (3/50). The eight comparison children who failed had some combination of strabismus (6/8), poor distance acuity (6/8) and/or poor near vision (3/8). Of the subset of 33 children who attended for comprehensive visual assessment, 13/22 OE children failed vs 2/11 comparison children. All 13 OE children who failed had strabismus, plus some combination of poor distance acuity (9/13), nystagmus (5/13), poor binocular vision (4/13) and/or poor near acuity (3/ 13). Nystagmus was always horizontal, manifest-latent with strabismus; eye movement recordings confirmed the waveform as in keeping with fusion maldevelopment nystagmus syndrome. The two comparison children who failed both had poor acuity; one also had strabismus. Monocular and binocular $120^{\prime}$ pattern-onset VEPs were present in all $30(20 \mathrm{OE}, 10$ comparison) children completing testing: while $\mathrm{CI}, \mathrm{CII}$ and CIII tended to be smaller and slower in OE children, the difference was not significant. Prenatal drug exposures for children who failed and who passed visual assessment were very similar.

Conclusion Long-term visual abnormalities were found in more than half of this cohort of OE children at age 8-10 years; the abnormalities indicate failure of binocular vision development. We postulate an opioid teratogenic effect causing loss of binocular connections within the striate cortex. Children affected by this fetal opioid syndrome are likely to be additionally challenged by socio-economic factors and other prenatal drug exposure.

Conflict of interest: None

Funding: Action for Medical Research, UK; Chief Scientist Office, Scottish Government

Award applications: None.

Corresponding author email: ruth.hamilton@glasgow.ac.uk

\section{O1.5 Paediatric P100 VEP reference ranges from three European medical centers}

Mikó-Baráth, Eszter ${ }^{1}$, Thompson, Dorothy A. ${ }^{2}$, Jandó, Gábor $^{1}$, Hamilton, Ruth ${ }^{3,4}$

${ }^{1}$ Institute of Physiology, Medical School, University of Pécs, Pécs, Hungary, ${ }^{2}$ The Tony Kriss Visual

Electrophysiology Unit, Clinical and Academic Department of Ophthalmology, Great Ormond Street Hospital for Children NHS Trust, London, UK, ${ }^{3}$ Department of Clinical Physics and Bioengineering, NHS Greater Glasgow \& Clyde, Glasgow, UK, ${ }^{4}$ College of Medical, Veterinary \& Life Sciences, University of Glasgow, Glasgow, UK

Purpose In order to establish age-specific reference ranges with $90 \%$ confidence limits for the ISCEV standard, pattern reversal VEPs were recorded in infants and children in three European medical centers. The main question of the study was whether differences in the experimental setups and the data acquisition systems between the centers influence P100 VEP latencies significantly.

Methods In a cross-sectional study, one Hungarian and two UK centers, using similar protocols, participated. Paediatric 
pattern reversal VEP data were collected for the two ISCEV recommended check sizes $\left(60^{\prime}\right.$ and $\left.15^{\prime}\right)$ from healthy controls. P100 VEP latencies from the centers were compared by twoway ANOVA to determine the statistical homogeneity of the three datasets. The VEP data were plotted against age, outliers were removed and the ages where data reached steady-state were determined for each check size. The pooled data set included 467 infants aged between 2 and 52 weeks and 187 children aged between 1 and 16 years. Age-specific reference ranges were established for the combined data set.

Results Since data, measured after the steady-state of the latency, were not significantly different from the three centers for the $60^{\prime}$ stimulus, VEP latencies could be considered homogenous. The P100 VEP latency of the combined data set showed a rapid drop during infancy which stabilized by the end of the 30th week for $60^{\prime}$ check size: thereafter, 90\% CI reference limits were between 90 and $112 \mathrm{~ms}$ (mean: $101 \mathrm{~ms}$ ). For $15^{\prime}$ check size, VEP latency stabilized by the 56th week, and thereafter reference limits were between 98 and $116 \mathrm{~ms}$, (mean: $107 \mathrm{~ms}$ ). For some infants, VEPs could not be recorded for $15^{\prime}$ check size in the first year.

Conclusion These preliminary findings suggest that data collected according to the ISCEV standards in different European electrophysiology laboratories can be combined into a larger reference data set, resulting in more reliable reference ranges and increased diagnostic power. Our dataset paves the way for national and international reference intervals for each ISCEV protocol.

Conflict of interest: None

Funding: Financial support: HUNGARIAN BRAIN RESEARCH PROGRAM 2.0 (NAP 2.0): 2017-1.2.1-NKP2017-00002 University of Pécs Medical School: KA-2019-25, FIKP 2.

Award applications: None.

Corresponding author email: eszter.miko-barath@aok.pte.hu

\section{O1.6 Flicker ERG in newborn term infants}

\section{Gerth-Kahlert, Christina ${ }^{1}$, Weber, Caroline ${ }^{2}$, Hanson,} James V.M ${ }^{1}$, Pfäffli, Oliver $A^{3}$, McCulloch, Daphne L. ${ }^{4}$

${ }^{1}$ Department of Ophthalmology, University Hospital Zurich and University of Zurich, Zurich, Switzerland; ${ }^{2}$ Department of Neonatology, University Hospital Zurich and University of Zurich, Zurich, Switzerland; ${ }^{3}$ Department of Ophthalmology, Cantonal Hospital Lucerne, Switzerland; ${ }^{4}$ School of Optometry and Vision Science, University of Waterloo, Waterloo, Canada

Purpose To develop and validate a flicker ERG protocol in termborn infants for potential future screening in pre-term infants who are at risk for developing retinopathy of prematurity.

Methods Design: non-randomized cross-sectional study at the University Hospital Zurich. Subjects: Neonates born at term (gestational age (GA): 37 to 41 6/7 weeks) without ocular or systemic malformations or significant postnatal complications were examined. Flicker ERG: Recordings were conducted within the first four days of life. Stimulation was delivered through closed eyelids with natural pupils while the neonates were asleep using the handheld RETeval ${ }^{\circledR}$ device with a custom-made protocol. The flicker stimulus series included $28.3 \mathrm{~Hz}$ flicker at 3, 6, 12, 30, and
$50 \mathrm{~cd} \cdot \mathrm{s} / \mathrm{m}^{2}$, and a step of $12 \mathrm{~cd} \cdot \mathrm{s} / \mathrm{m}^{2}$ delivered at $14.2 \mathrm{~Hz}$ at the end of the recording. ERG data were collected using disposable skin electrodes placed below the eyes. Eye position was verified prior to each step of recording to ensure that the eyes had not rotated upwards. Two measurements per stimulus level were performed. Analysis: After viewing the curves and verifying reproducibility of the responses, custom-written code run in MatLab was used to extract flicker ERG peak times and amplitudes from the averaged measurements for each stimulus level for each subject.

Results 28 term-born neonates, with females and males almost evenly distributed, were included in the analysis. All neonates tolerated the testing procedure very well. Flicker ERG recording was possible in all subjects; however responses to the weaker stimuli were not found in all neonates (with detectable ERGs in 20/28, 25/28, and 27/28 at 3, 6, and $12 \mathrm{~cd} \cdot \mathrm{s} / \mathrm{m}^{2}$, respectively). Flicker ERG amplitudes increased and implicit times decreased with increasing stimulus strength, with amplitude increases being particularly prominent for the 30 and $50 \mathrm{~cd} \cdot \mathrm{s} / \mathrm{m}^{2}$ conditions relative to the three lower levels.

Conclusion Flicker ERG recording is possible in sleeping neonates through closed eyelids using skin electrodes and without mydriasis. Responses were detectable even at low stimulus intensities in the majority of tested infants. Further studies will provide information about the feasibility and applicability of this protocol in premature infants.

Conflict of interest: None

Funding: Velux Foundation

Award applications: None.

Corresponding author email: christina.gerth-kahlert@usz.ch

\section{Oral Session 2: Visual Electrophysiolgy in Monitoring Disease Process/Treatment}

O2.1 The focal macular photopic negative response from eyes with macula-on rhegmatogenous retinal detachment: comparison between the values measured from the baseline and from the peak of the $b$-wave

Akima, Kunihiko $^{1,2}$, Fujinami, Kaoru ${ }^{1,2,3,4}$, Watanabe, Ken $^{1,2}$,Noda, Toru ${ }^{1,2}$, Miyake, Yozo ${ }^{2,5,6}$, Tsunoda, Kazushige $^{1,2}$

${ }^{1}$ Department of Ophthalmology, National Hospital Organization Tokyo Medical Center, Tokyo, Japan, ${ }^{2}$ National Institute of Sensory Organs, National Hospital Organization Tokyo Medical Center, Tokyo, Japan, ${ }^{3}$ Department of Ophthalmology, Keio University, School of Medicine, Tokyo, Japan, ${ }^{4}$ UCL Institute of Ophthalmology, London, UK, ${ }^{5}$ Aichi Medical University, Aichi, Japan,

${ }^{6}$ Next Vision, Kobe Eye Center, Kobe, Japan

Purpose There are two major ways to measure the photopic negative response $(\mathrm{PhNR})$ : the difference from the baseline to the trough (BT) and from the peak of the b-wave to the trough (PT). We assess the two methods for the PhNR measurement in focal macular electroretinography (FMERG) recorded from eyes with macula-on rhegmatogenous retinal detachments (RRDs), by comparing with the alteration of a-, b-waves and oscillatory potentials (OPs) in the affected eyes. 
Methods The FMERG was recorded from the affected eye and the fellow eye of patients diagnosed with macula-on RRD. The applied procedures were the same as described previously [Akiyama et al., 53rd-57th ISCEV Symposium (2015-2019)]. The protocol of the FMERG recording was as follows: (i) luminance (stimulus/background): $115.7 / 8.0 \mathrm{~cd} / \mathrm{m}^{2}$; (ii) stimulus duration: $100 \mathrm{~ms}$; (iii) spot size: 15 degrees in diameter; and (iv) average at least 300 sweeps. The amplitudes and the implicit times of the a-wave, b-wave, OPs, and PhNR were measured. The amplitude of the PhNR was measured by the two methods (BT and $\mathrm{PT}$ ). A ratio of PhNR to b-wave was also calculated for each of the methods. For statistical analyses, each parameter was compared between the affected and fellow eyes using a paired $t$ test.

Results Thirty cases (mean age \pm standard deviation, $50.7 \pm 8.8$ years) were included. There was no statistical difference in refraction or visual acuity between the affected and fellow eyes. The mean amplitude and implicit time of the affected/fellow eyes were $1.23 \pm 0.49 \mu \mathrm{V} / 1.53 \pm 0.54 \mu \mathrm{V}$ and $20.09 \pm 1.16 \mathrm{~ms} / 20.08 \pm 1.00 \mathrm{~ms}$ for a-wave; $3.36 \pm$ $1.00 \mu \mathrm{V} / 4.59 \pm 1.05 \mu \mathrm{V}$ and $38.65 \pm 2.32 \mathrm{~ms} / 38.84 \pm 2.39$ for b-wave; and $0.72 \pm 0.23 \mu \mathrm{V} / 0.87 \pm 0.26 \mu \mathrm{V}$ and $32.01 \pm 1.19 \mathrm{~ms} / 31.83 \pm 1.16 \mathrm{~ms}$ for OPs. The mean amplitude of PhNR (BT) and PhNR (PT) of the affected/fellow eyes was, respectively, $0.18 \pm 0.91 \mu \mathrm{V} /-0.03 \pm 1.12 \mu \mathrm{V}$ and $2.31 \pm 1.03 \mu \mathrm{V} / 3.03 \pm 1.06 \mu \mathrm{V}$. The mean implicit time of the PhNR of the affected/fellow eyes was $57.20 \pm 5.19 \mathrm{~ms} /$ $57.43 \pm 3.63 \mathrm{~ms}$. The PhNR to b-wave ratio for the BT and PT measurements was $0.06 \pm 0.27 / 0.028 \pm 0.27$ and $0.68 \pm 0.18$ / $0.67 \pm 0.19$. Statistically significant differences between the affected and fellow eyes were shown in terms of the amplitudes of a-wave, b-wave, OPs, and PhNR (PT) $(p<0.001$ for each of them), but not in PhNR (BT) $(p=0.160)$. Statistical difference was not observed in the implicit time of any of these components nor the PhNR to b-wave ratio of both measurements.

Conclusion The PhNR (PT) was reduced in the affected eyes compared to the fellow eyes in parallel with the reduced amplitudes in other components, while the PhNR (BT) showed no difference between the affected and fellow eyes. This discrepancy occurred probably due to the varied extent of influence on each of the measurements by the succeeding offresponses and/or other factors derived from the relatively long stimulus duration (100 ms).

Conflict of interest: None.

Funding: None.

Award applications: Marmor Award.

Corresponding author email: akiyamakunihiko@

kankakuki.go.jp

O2.2 Electrophysiology as a prognostic indicator of visual recovery in diabetic patients undergoing cataract surgery

Wang, Hao $^{1,2}$, Li, Fuliang ${ }^{1,2}$, Li, Jiawen ${ }^{1,2}$, Lin, Jun ${ }^{1,2}$, Liu, Meifang ${ }^{1,2}$, Wang, Gang ${ }^{1,2}$, Wang, Min ${ }^{1,2}$, Ran, Li ${ }^{1,2}$, Robson, Anthony G. ${ }^{3,4}$, Li, Shiying ${ }^{1,2}$

${ }^{1}$ Southwest Hospital/Southwest Eye Hospital, Third Military Medical University (Army Military Medical University), Chongqing, China; ${ }^{2}$ Key Lab of Visual Damage and Regeneration \& Restoration in Chongqing.
Chongqing, China; ${ }^{3}$ Department of Electrophysiology, Moorfields Eye Hospital, London, UK; ${ }^{4}$ Institute of Ophthalmology, University College London, London, UK

Purpose Visual outcomes after cataract surgery in diabetic patients with retinal or visual pathway disease are difficult to predict as the fundus may be obscured, and assessment of visual potential is challenging. This study assessed the value of visual electrophysiology as a prognostic indicator of visual recovery in diabetic patients with cataract, prior to cataract surgery.

Methods Forty-one diabetic patients (aged 50-80; 74 eyes) and 13 age-matched non-diabetic control patients (21 eyes) were examined prior to cataract surgery. Pre-surgical examinations included best-corrected visual acuity (BCVA), slit-lamp biomicroscopy, ISCEV-standard full-field electroretinography (ERG) and flash visual evoked potential (flash VEP) testing. Electrophysiological assessments included quantification of the DA 3.0 ERG oscillatory potentials (OPs; OP1, OP2, OP3, OP4) and flash VEP P1, P2 and P3 components. Post-operative BCVA was measured in all cases and the diabetic patients grouped according to the severity of visual acuity loss; mild $(\log$ MAR $\leq 0.1)$, moderate $(0.1<\log$ MAR $<0.5)$ or severe $(\log$ MAR $\geq 0.5)$. A fourth group included those without diabetes. The pre-surgical electrophysiological data was compared between the four groups by analysis of variance.

Results The severity of post-surgical visual acuity loss in the diabetic patients was mild $(n=22)$, moderate $(n=31$ eyes $)$ or severe $(n=21$ eyes). In the group without diabetes, postsurgical visual impairment was mild $(n=21$ eyes). The preoperative DA 10.0 ERG a-wave amplitude, DA 3.0 ERG OP2 amplitudes and the LA 3.0 a- and b-wave amplitudes showed significant differences between the four groups. There were significant differences in the amplitude of flash VEP P2 component among diabetic groups.

Conclusion Electrophysiological assessment of patients with cataract can provide a useful measure of retinal and visual pathway function. Full-field ERG components, including the DA 10.0 ERG a-wave, DA 3.0 ERG OP2 and the LA 3.0 a- and b-wave amplitudes are of prognostic value in predicting postsurgical visual acuity and may inform the surgical management of diabetic patients with cataract.

Conflict of interest: None.

Funding: Natural Science Foundation of Chongqing (cstc2017jcyjAX0111), the National Natural Science Foundation of China (NSFC 81900902, 81974138), National Basic Research Program of China (2018YFA0107301), and the Natural Science Foundation of Chongqing (cstc2017shmsA130100)

Award applications: None.

Corresponding author email: shiying_li@126.com

O2.3 Role of multifocal electroretinogram in assessment of early retinal dysfunction in hypertensive patients

Farouk, Ayat Allah Hussein ${ }^{1}$, Elhadidy, Reem ${ }^{1}$ Abd EISalam, Eman Attia ${ }^{1}$, Zedan, Rasha ${ }^{2}$, Azmy, Radwa ${ }^{1}$

${ }^{1}$ Clinical neurophysiology, Cairo University, Cairo, Egypt, ${ }^{2}$ Department of Opthalmology- Faculty of Medicine- Cairo University 
Purpose To investigate localized retinal dysfunction in hypertensive patients using the multifocal electroretinogram (mfERG) and to assess its sensitivity as an early predictor for the development of retinopathy in hypertensive patients.

Methods Ninety-eight eyes were included in this case-control study. Twenty-eight eyes of healthy subjects served as a control group (group I). Seventy eyes belonged to patients with systemic hypertension assigned into two groups: group II included 39 eyes of hypertensive patients with normal fundus and group III included 31 eyes of patients with signs of hypertensive retinopathy. All participants were subjected to complete ophthalmic and electrophysiological examination using mfERG. N1 and P1 amplitudes and implicit times from the central hexagon and four concentric rings across the visual field were analyzed

Results mfERG amplitudes were significantly reduced in the hypertensive group with retinopathy compared to controls. N1 amplitude was significantly reduced in the most eccentric ring in eyes of hypertensive patients with normal fundus.

Conclusion mfERG is a sensitive objective tool for assessment of retinal dysfunction in hypertensive patients. mfERG amplitude is a promising predictor for early development of retinopathy in systemic hypertension.

Conflict of interest: None.

Funding: None.

Award applications: Marmor Award.

Corresponding author email: aia.farouk@yahoo.com.

O2.4 Macular assessment using ERG and spectral domain optical coherence tomography (SD-OCT) following silicone oil removal (SOR) in eyes operated for retinal detachment (RD)

\section{Goel, Neha ${ }^{1}$, Shukla, Gaurav ${ }^{2}$, Jain, Prashant ${ }^{2}$ \\ ${ }^{1}$ Department of Vitreoretina, Eye, 7 Chaudhary Eye Centre, Daryaganj, New Delhi, India; ${ }^{2}$ ICARE Eye Hospital and Postgraduate Institute, Noida, U.P., India}

Purpose To study the structural (using SD-OCT) and functional (using PERG and mfERG) changes in the macula following silicone oil removal (SOR) in patients operated for RD.

Methods Prospective interventional study on 32 eyes of 32 patients who underwent pars plana vitrectomy with Silicon oil injection $(\mathrm{SiO})$ for rhegmatogenous $\mathrm{RD}$ and had attached retina following surgery. Parameters noted pre- and 1 month postSOR included best corrected visual acuity (ETDRS chart), IOP, fundus photography, SD-OCT (central foveal thickness and mean macular thickness), PERG and mfERG (RETI Port/Scan 21, Roland Consult, Germany using DTL electrodes, as per ISCEV guidelines). Quantitative variables were compared using the Wilcoxon signed rank test. Pearson correlation coefficient/spearman rank correlation coefficient was used to assess correlation between quantitative variables.

Results Mean age of the patients was $52.72 \pm 17.1$ years; 16 were males and 16 were females. Time interval between primary surgery and SOR ranged from 90 to 455 days. BCVA improved significantly from $0.66 \pm 0.19$ to $0.46 \pm 0.23$ logMAR units following SOR $(p<0.0001)$. Macular thickness on SD-OCT did not show any significant change post SOR. On PERG, N95 and P50 amplitudes improved significantly following $\operatorname{SOR}(p=0.0004$ and 0.001 , respectively). On mfERG, P1 and N1 amplitudes increased significantly in rings 1-5. No significant correlation was found between the time interval before SOR and any of the parameters on SD-OCT, PERG or mfERG.

Conclusion SOR increases amplitudes on PERG and mfERG and improves BCVA after retinal reattachment. These changes maybe attributable to the insulating effect of silicone oil on the retina rather than toxicity. PERG and mfERG can be used to evaluate silicone oil induced macular dysfunction. Removal of silicone oil is recommended for better visual acuity and early recovery of electroretinographic potentials of retina, seen in PERG and mfERG, as soon as a stable environment of the retina is achieved.

Conflict of interest: None

Funding: None

Award applications: None.

Corresponding author email: nehadoc@hotmail.com

O2.5 Combined multi-modal assessment of glaucomatous damage with electroretinography and optical coherence tomography/angiography

Al-Nosairy, Khaldoon O. ${ }^{1}$, Prabhakaran, Gokulraj ${ }^{1}$, Pappelis, Konstantinos ${ }^{2}$, Thieme, Hagen ${ }^{1}$, Hoffmann, Michael B. ${ }^{1}$

${ }^{1}$ Ophthalmology Department, Otto von Guericke University, Magdeburg, Germany; ${ }^{2}$ Department of Ophthalmology, University of Groningen, University Medical Center, Groningen, The Netherlands

Purpose To compare the diagnostic performance and to evaluate the interrelationship of electroretinographic, structural, and vascular measures in glaucoma.

Methods For 14 eyes of 14 healthy controls and 15 eyes of 12 patients with glaucoma ranging from preperimetric to advanced stages, OCT, OCT-A, and electrophysiological measures [multifocal photopic negative response ratio $(\mathrm{mfPhNR})$ and steady state pattern electroretinogram (ssPERG)] were obtained to assess changes in retinal structure, microvasculature, and function, respectively. Diagnostic performance was assessed via area-under-curve (AUC) measures obtained from ROC analyses. The interrelation of the different measures was assessed with correlation analyses.

Results mfPhNR and ssPERG amplitudes, parafoveal (pfVD) and peripapillary vessel density (pVD), macular ganglion cell inner plexiform layer thickness (mGCIPL) and peripapillary retinal nerve fibre layer thickness (pRNFL) were significantly reduced in glaucoma. The AUC for $\mathrm{mfPhNR}$ was highest among diagnostic modalities (AUC: 0.88, 95\%; CI: 0.75-1.0, $p<0.001$ ), albeit not statistically different from that for macular (mGCIPL: 0.76, 0.58-0.94, $p<0.05$; pfVD: 0.81, $0.65-0.97, p<0.01$ ) or peripapillary imaging (pRNFL: 0.85 , $0.70-1.0, p<0.01$; pVD: $0.82,0.68-0.97, p<0.01$ ). Combined functional/vascular measures yielded the highest AUC (mfPhNR-pfVD: $0.94,0.85-1.0, p<0.001$ ). The functional/ structural measure correlation (mfPhNR-mGCIPL correlation 
coefficient (rs): $0.58, p=0.001$; mfPhNR-pRNFL rs: 0.66 , $p<0.0001)$ was stronger than the functional-vascular correlation (mfPhNR-pfVD rs: 0.29, $p=0.13$; mfPhNR-pVD rs: $0.54, p=0.003)$.

Conclusion The combination of ERG measures and OCT-A improved diagnostic performance in glaucoma. Combing ERG, structural and OCT-A parameters provides an enhanced understanding of the pathophysiology of glaucoma.

Conflict of interest: None.

Funding: This work was supported by European Union's Horizon 2020 research and innovation programme under the Marie Sklodowska-Curie grant agreement (No. 675033) and by funding of the German research foundation (DFG; HO2002/201) to $\mathrm{MBH}$.

Award applications: None.

Corresponding author email: michael.hoffmann@ med.ovgu.de

O2.6 Correlation between OCT-angiography and photopic negative response in patients with primary open angle glaucoma

\author{
Abdelshafy, Marwa ${ }^{1}$, Abdelshafy, Ahmed ${ }^{1}$ \\ Ophthalmology Department, Benha Faculty of Medicine, \\ Benha University, Egypt
}

Purpose To assess the correlation between vascular, structural and functional changes of the peripapillary retinal nerve fiber (RNFL) and ganglion cell complex (GCC) in patients with open angle glaucoma (OAG) using OCT-angiography (OCTA) and photopic negative response (PhNR).

Methods In this prospective cross-sectional comparative study, 152 eyes of 76 patients were included: 72 eyes of 36 patients with OAG with a mean age of $40.2 \pm 4.39$ years (Glaucoma group) and 80 eyes of 40 healthy subjects with a mean age of $40.5 \pm 4.02$ years (control group). All participants underwent full ophthalmological examination, optical coherence tomography (OCT), OCTA using RTVue XR OCT Avanti System AngioVue Version 201.2.0.93 (Optovue, Fremont, CA, USA) and $\mathrm{PhNR}$ measurement using RETI-port/scan 21(Roland Consult, Brandenburg, Germany). RNFL, GCC thickness and the amplitude and implicit time of PhNR were measured. $6 \mathrm{~mm} \times 6 \mathrm{~mm}$ macular OCTA scans were used to measure superficial capillary plexus vessel density (SCP-VD) and deep capillary plexus vessel density (DCP-VD). $4.5 \mathrm{~mm} \times 4.5 \mathrm{~mm}$ optic disc head OCTA scans were used to measure the peripapillary vessel density (RPC-VD). Statistical analysis was performed to compare GCC, RNFL thickness, OCTA and PhNR values between groups.

Results GCC and RNFL (median IQR) thicknesses were reduced in OAG versus normal $(81.5 \mu \mathrm{m}$ vs $99 \mu \mathrm{m}, p<0.001$; $90 \mu \mathrm{m}$ vs $108.5, p<0.001$ respectively). In the glaucoma group the superior and inferior quadrants of RNFL were significantly thinner. There were significant differences of PhNR amplitude and latency between the two groups $(p<0.001)$. SCP-VD, DCP-VD and RPC-VD were reduced in OAG versus normal (47.85 vs 54.8, $p<0.001 ; 47.57$ vs $57.25, p<0.001 ; 48.15$ vs $59.8, p<0.001$, respectively), with a trend of worsening with increased OAG severity. Reduced
PhNR amplitude and prolonged latency were associated with reduced SCP-VD, DCP-VD and RPC-VD $\left(p<0.001 ; \mathrm{R}^{2}\right.$ $=0.725, \quad 0.904,0.875,-0.854,-0.874$ and -0.940 , respectively).

Conclusion The glaucomatous eyes had significant reduction of the macular ganglion cell-inner plexiform layer (GCIPL) and peripapillary RNFL microcirculation. There was a significant association of the vessel parameters with functional and structural measurements. Regarding functional correlations of OCTA vascular parameters, the SCP-VD, DCP-VD and RPCVD had significant positive correlation with PhNR amplitude and significant negative correlation with $\mathrm{PhNR}$ latency. Vascular dysfunction plays a significant role in glaucoma pathogenesis. The vascular OCTA parameters and PhNR (an objective measure of ganglion cell function) can supplement the structural OCT measurement for diagnosis and monitoring OAG, especially in cases with atypical optic nerves such as those in high myopes.

Conflict of interest: None.

Funding: None.

Award applications: None.

Corresponding author email: drmarwatab12012@gmail.com

\section{Oral Session 3: Clinical Electroretinography: Stimulus and Recording Parameters}

\section{O3.1 The effect of pupil size on the photopic negative response}

Zueva, Marina ${ }^{1}$, Tsapenko, Irina ${ }^{1}$, Kotelin, Vladislav', Kirillova, Maria ${ }^{1}$, Zhuravleva, Anastasia ${ }^{1}$, Bessmertnyi, Alexander ${ }^{1}$

Helmholtz National Research Center of Eye Diseases, Moscow, Russia

Purpose The ISCEV Standard recommends dilating the pupils before performing an ERG, however sometimes there is a need to record the ERG with a natural pupil diameter when it is impossible to dilate, in specific contraindications, and for ethical reasons in testing healthy controls. We aimed to evaluate the effect of pupil size on the photopic negative response $(\mathrm{PhNR})$.

Methods Twelve healthy individuals (23 eyes) aged 24 to 40 years were examined. Refractive error did not exceed 0.5 diopters. PhNR was evaluated in the ERG recorded on a blue background to red flashes $\left(0.375,0.75,1.5,3.0 \mathrm{~cd} \mathrm{~s} / \mathrm{m}^{2}\right)$ with RETIport/scan21 (Roland Consult) according to ISCEV protocols. First, the ERG was recorded with a natural pupil diameter $(3.5 \pm 0.2 \mathrm{~mm})$. Then, the recording was repeated in pharmacological mydriasis $(8.7 \pm 0.1 \mathrm{~mm})$.

Results In eyes with a natural pupil, compared with druginduced mydriasis, the amplitude of $\mathrm{PhNR}$ was significantly reduced in the ERG to the weakest stimulus $\left(0.375 \mathrm{~cd} \mathrm{~s} / \mathrm{m}^{2}\right)$. The effect of pupil size on PhNR was practically absent in responses to medium and higher strength flashes. This phenomenon can be associated with an uneven distribution of light over the retina with small pupil sizes and is maximal in the responses to weak flashes. For the maximal strength flash (standard $3.0 \mathrm{~cd} \mathrm{~s} / \mathrm{m}^{2}$ ), changes were revealed only for the 
amplitude of the b-wave and PhNR from the b-wave peak, which may reflect a decrease in the number of stimulated photoreceptors during the ganzfeld stimulation when the pupil is not dilated. There was no difference in the index $\mathrm{PhNR} / \mathrm{b}$ in eyes with dilated and natural pupil,.

Conclusion When it is necessary to record PhNR without mydriasis, especially in screening testing, we recommend limiting the protocol to recording PhNR at standard (highest) flash strength and use the index with low variability: the amplitude ratio of $\mathrm{PhNR} / \mathrm{b}$ instead of the absolute amplitude of PhNR.

Conflict of interest: None.

Funding: None.

Award applications: None.

Corresponding author email: visionlab@yandex.ru

O3.2 Effects of recording sequence on flicker ERGs recorded with natural pupils corrected for pupil area

Kondo, Mineo', Kato, Kumiko', Sugawara, Asako ${ }^{1}$, Ikasugi, Kengo ${ }^{1}$, Matsubara, Hisashi ${ }^{1}$, Sugimoto, Masahiko', Nagashima, Ryunosuke', McCulloch, Daphne ${ }^{2}$

${ }^{1}$ Ophthalmology, Mie University, Tsu, Japan; ${ }^{2}$ School of Optometry and Vision Science, University of Waterloo, Waterloo, Canada

Purpose A new hand-held electroretinographic (ERG) recording system can record ERGs without mydriasis. However, this RETeval system cannot record ERGs from both eyes simultaneously. The purpose of this study was to determine whether the sequence of the ERG recordings will alter the results.

Methods We studied 30 eyes of 30 healthy subjects. Flicker ERGs were recorded with the RETeval system without mydriasis and were elicited by 8,16 , and 32 photopic Td-s. The flicker ERGs were recorded at two sessions. In Session 1, the ERGs were recorded from the right eye and then the left eye; and in Session 2, ERGs were recorded from the left eye and then the right eye. We compared the implicit times, amplitudes, and pupillary diameters of the right eye between these two sessions.

Results The implicit time of the flicker ERGs was significantly shorter $(p<0.001)$ and the pupil diameters were significantly smaller $(p=0.013)$ at Session 2 than Session 1 but only for the lower stimulus intensity of $8 \mathrm{Td}$-s. There was a significant correlation of the differences in the implicit times and the differences in the pupil diameter between the two sessions ( $\mathrm{R}=0.406, p=0.026)$.

Conclusion The results indicate that the implicit times of the fundamental components of RETeval flicker ERGs can be affected by the sequence of recordings for lower stimulus intensities, most likely due to the differences of the pupillary diameter during the recordings. We recommend that stronger stimuli be used to record the RETeval flicker ERGs to minimize the effect of sequence of recordings.

Conflict of interest: None.

Funding: None.

Award applications: None.

Corresponding author email: mineo@clin.medic.mie-u.ac.jp
O3.3 Rod-driven electroretinogram responses in moderate Vitamin A deficiency are consistent with desensitisation due to an equivalent background

Margetts, Megan ${ }^{1,2}$, Jiang, Xiaofan ${ }^{2,3}$, Rufus-Toye, Remi $^{1,4}$, Chow, Isabelle ${ }^{3}$, Indusegaran, Mathura ${ }^{5}$, Webster, Andrew $\mathbf{R}^{2,6}$, Hammond, Christopher $\mathbf{J}^{5}$, Mahroo, Omar $A^{5,7,8,9}$

${ }^{1}$ Physiology, Development and Neuroscience, University of Cambridge, Cambridge, UK; ${ }^{2}$ Institute of Ophthalmology, University College London, London, UK; ${ }^{3}$ Section of Ophthalmology, King's College London, St Thomas' Hospital Campus, London, UK; ${ }^{4}$ Moorfields Eye Hospital, London, UK

Purpose Typically, in Vitamin A deficiency, rod system responses are undetectable, whilst cone system responses are preserved. Reduction in sensitivity is often attributed to reduction in quantal catch, but the effect of unregenerated opsin in activating the phototransduction cascade is likely to be relevant. We investigated this using dim-flash electroretinograms (ERGs), recorded in the presence of a range of dim backgrounds, in healthy subjects and in a single patient with moderate and severe vitamin A deficiency.

Methods ERGs were recorded from three healthy adults (after pharmacological mydriasis) in response to white LED flashes ( 0.001 to $30 \mathrm{~cd} \mathrm{~s} / \mathrm{m}^{2}$ ), using conductive fibre electrodes (lower conjunctival fornix). Flashes were delivered in the darkadapted state and in the presence of blue backgrounds of varying strength $\left(0.03,0.1,1.0,3.0,10,30 \mathrm{~cd} \mathrm{~s} / \mathrm{m}^{2}\right)$. For this study, analysis was restricted to dim flashes (originating in roddriven bipolar cells). Recordings were also made from one patient with vitamin A deficiency at different times: with severe vitamin A deficiency; with moderate vitamin A deficiency $(0.58$ micromol/L), and after Vitamin A repletion ( $2.7 \mathrm{micromol} / \mathrm{L})$.

Results In healthy participants, as dim-flash strength increased in the dark, b-wave amplitudes increased and peak times became shorter (from c. $125 \mathrm{~ms}$ for a $0.001 \mathrm{~cd} \mathrm{~s} / \mathrm{m}^{2}$ flash to 50-60 ms for a $0.1 \mathrm{~cd} \mathrm{~s} / \mathrm{m}^{2}$ flash). As dim background strength increased, the b-wave for a given strength dim flash reduced in amplitude and peak times became shorter. The patient showed the same relationship after Vitamin A repletion. When moderately deficient, the dim-flash b-wave was reduced in amplitude but of shorter peak time (resembling the responses from the healthy participants obtained to the same strength flash delivered in the presence of a dim background). When severely deficient, the dim-flash response was undetectable.

Conclusion Compared with the b-wave elicited by a given dim-flash strength in the dark, responses to the same flash delivered in the presence of a dim background are smaller and of shorter peak time, whilst responses to a weaker flash in the dark are also smaller but of longer peak time. Responses in moderate vitamin A deficiency mimic the effect of a background (rather than resembling responses to weaker flashes). This is consistent with the mechanism of desensitisation being an "equivalent background" (probably due to unregenerated opsin) rather than a simple reduction in quantal catch.

Conflict of interest: None

Funding: Wellcome Trust 
Award applications: None.

Corresponding author email: o.mahroo@ucl.ac.uk

O3.4 Derived rod and cone system flash electroretinogram responses from 208 adult twins: relationships with age and heritability

Jiang, Xiaofan ${ }^{1,2,3}, \mathrm{Xu}, \mathrm{Zihe}^{2,3}$, Williams, Katie $\mathbf{M}^{1,2,4}$, Webster, Andrew $\mathbf{R}^{1,5}$, Hysi, Pirro $\mathbf{G}^{2,3}$, Hammond, Christopher $\mathbf{J}^{2,3}$, Mahroo, Omar $A^{1,2,3,4,5,6}$

${ }^{1}$ Institute of Ophthalmology, University College London, London UK; ${ }^{2}$ Section of Ophthalmology, King's College London, St Thomas' Hospital Campus, London, UK; ${ }^{3}$ Department of Twin Research and Genetic Epidemiology, King's College London, St Thomas' Hospital Campus, London UK; ${ }^{4}$ Moorfields Eye Hospital, London, UK; ${ }^{5}$ Physiology, Development \& Neuroscience, University of Cambridge, Cambridge UK

Purpose Human rod signals are routinely investigated by analysing electroretinogram (ERG) responses to flashes delivered in the dark, but these responses contain cone contributions. One method for removing the cone contribution is by subtraction of responses to the same flashes delivered on a dim rod-saturating background. Such a method was employed in a large twin cohort. We present correlations with age and estimates of heritability.

Methods ERGs were recorded from 208 adult twins (after pharmacological mydriasis), using conductive fibre electrodes, in response to white xenon flashes $(0.67,4.0,13$ and 67 photopic $\mathrm{cd} \mathrm{s} / \mathrm{m}^{2}$ ), delivered in the dark (following $20 \mathrm{~min}$ dark adaptation) and also in the presence of a dim blue rodsaturating background (1.0 scotopic $\mathrm{cd} / \mathrm{m}^{2}$ and 30 photopic $\mathrm{cd} /$ $\mathrm{m}^{2}$ ). The latter responses gave an estimate of the (minimally light-adapted) cone system response. Subtraction of these responses from the former yielded an estimated dark-adapted rod system response. Responses were averaged from several flash presentations, and ERG parameters were averaged from both eyes. Coefficients of intra-pair correlation were calculated for monozygotic and dizygotic twins and heritability estimated using structural equation modelling.

Results Mean (SD) age was 62.5 (11.5) years; 93\% were female. All ERG a-wave amplitudes (both for rod and cone systems) increased, while peak times became shorter, with increasing stimulus strength. Cone-driven b-waves (amplitudes and peak times) peaked at an intermediate intensity (representing the photopic hill); b-waves of estimated rod responses changed little with increasing stimulus strength. ERG amplitudes were negatively correlated with age. Correlations for peak times were positive. For rod and cone system responses, respectively, the strongest correlations with age were for the a-wave peak time elicited by the strongest flash and for the b-wave peak time elicited by the weakest flash. Monzygotic correlations were higher than dizygotic correlations for all parameters, and point estimates of heritability ranged from 0.52 to 0.89 .

Conclusion Both derived rod and cone system parameters showed significant changes with age. Rod and cone response parameters also showed significant heritability, indicating that a large proportion of the variance in both rod and cone-driven responses are attributable to genetic factors. The derived rod responses may still be over-estimates due to the possibility of mild cone system desensitisation by the blue background.

Conflict of interest: None

Funding: Wellcome Trust, Moorfields Eye Charity, Fight for Sight UK

Award applications: None.

Corresponding author email: o.mahroo@ucl.ac.uk

O3.5 The importance of flicker electroretinogram in the course of birdshot chorioretinitis

Faber, Hanna ${ }^{1}$, Strasser, Torsten ${ }^{2}$, Kempf, Melanie ${ }^{3}$, Zierhut, Manfred $^{1}$, Deuter, Christoph ${ }^{4}$, Doycheva, Deschka $^{4}$, Stingl, Katarina ${ }^{4,5}$

${ }^{1}$ University Eye Hospital, Center for Ophthalmology, Eberhard Karls University, Tuebingen, Germany, ${ }^{2}$ Zrenner Lab, Institute for Ophthalmic Research, Center for Ophthalmology, Eberhard Karls University, Tuebingen, Germany, ${ }^{3}$ Institute for Ophthalmic Research, Center for Ophthalmology, Eberhard Karls University, Tuebingen, Germany, ${ }^{4}$ University Eye Hospital, Center for Ophthalmology, Eberhard Karls University, Tuebingen, Germany, ${ }^{5}$ Center for Rare Eye Diseases, Eberhard Karls University, Tuebingen, Germany

Purpose Earlier publications indicate that the implicit time of the photopic $31 \mathrm{~Hz}$ flicker ERG might be a sensitive marker of active inflammation in birdshot chorioretinitis. On a larger cohort, we evaluated whether scotopic $9 \mathrm{~Hz}$ and photopic $31 \mathrm{~Hz}$ flicker full-field electroretinography can discriminate active and stable disease phases in patients suffering from birdshot chorioretinitis.

Methods Chart review of 41 patients with birdshot chorioretinitis treated at the University Eye Hospital Tuebingen. Patients with co-morbidities with known effects on the ERG were excluded. BCR patients were included in the analysis if full-field ERG recordings of at least one active and one stable episode of birdshot chorioretinitis were available. Mean implicit time and amplitude of the scotopic $9 \mathrm{~Hz}$ and photopic $31 \mathrm{~Hz}$ Flicker were compared in the active and stable phase (paired t-test).

Results Mean implicit time did not differ between active and stable phase of birdshot chorioretinitis for the $9 \mathrm{~Hz}$ Flicker of the left eye $(p(\mathrm{OS})=0.68)$ and the $31 \mathrm{~Hz}$ Flicker of both eyes $(p(\mathrm{OS})=0.61), p(\mathrm{OD})=0.17)$. However, comparison of the $9 \mathrm{~Hz}$ flicker implicit times of the right eye in both disease states showed significance $(p(O D)=0.017)$. We could not see a significant alteration in the mean amplitude between active and stable disease in the $9 \mathrm{~Hz}$ Flicker $(p(\mathrm{OS})=0.40)$, $p(\mathrm{OD})=0.79)$ and the $31 \mathrm{~Hz}$ Flicker $(p(\mathrm{OS})=0.62)$, $p(\mathrm{OD})=0.71)$.

Conclusion The scotopic and photopic flicker full-field ERG might not be sensitive enough to monitor active inflammation of birdshot chorioretinitis. Although we found a significant difference in the $9 \mathrm{~Hz}$ flicker of eyes with active inflammation and stable phase on OD, the side difference cannot be explained. Multimodal diagnostic and careful clinical 
examination is necessary to decide about the presence of an active disease in birdshot chorioretinitis.

Conflict of interest: None

Funding: German Research Foundation (DFG):SFB1233, Robust Vision: Inference Principles and Neural Mechanisms, TP14

Award applications: None.

Corresponding author email: hanna.faber@med.unituebingen.de

O3.6 Growth in cone-driven ERG amplitudes with light adaptation in healthy adults and in patients with selected retinal diseases

Xu, Zihe ${ }^{1,2}$, Jiang, Xiaofan ${ }^{1,2}$, Bellingham, James ${ }^{2}$ Arno, Gavin ${ }^{2,3}$, Plant, Gordon $\mathbf{T}^{3}$, Webster, Andrew $\mathbf{R}^{2,3}$, Hammond, Christopher $\mathbf{J}^{1,4}$, Hysi, Pirro G ${ }^{1,4}$, Mahroo, Omar $A^{1,2,3,4}$

${ }^{1}$ Section of Ophthalmology, King's College London, St Thomas' Hospital Campus, London, UK; ${ }^{2}$ Institute of Ophthalmology, University College London, London, UK; ${ }^{3}$ Moorfields Eye Hospital, London, UK; ${ }^{4}$ Department of Twin Research and Genetic Epidemiology, King's College London, St Thomas' Hospital Campus, London, UK

Purpose The growth in amplitude of the cone-driven flash electroretinogram (ERG) over several minutes after onset of a photopic background following prior dark adaptation has been termed the Light Adaptation Effect. The mechanisms driving this are not known. A change in potential over the retinal pigment epithelium (RPE) occurs over a similar time course. We investigated the Light Adaptation Effect in healthy adults and in patients with selected retinal diseases to explore its kinetics and possible neuronal basis.

Methods Participants were dark adapted for $20 \mathrm{~min}$ and then exposed to the standard ISCEV photopic white background $\left(30 \mathrm{~cd} / \mathrm{m}^{2}\right)$. White flashes $\left(3 \mathrm{~cd} \mathrm{~s} / \mathrm{m}^{2}\right)$ were delivered (groups of 36 flashes every $2 \mathrm{~min}$ ) for $20 \mathrm{~min}$. B-wave amplitude was plotted as a function of time. Healthy adults were recruited as well as patients with specific retinal impairments.

Results In the healthy subjects $(n=8$; mean age 26 years; range 21-39 years), b-wave amplitudes approximately doubled (median increase 90\%), over the recording period. Most of the increase occurred in the first $10 \mathrm{~min}$, after which there was a relative plateau. The patients tested were as follows: a 23 year old patient with Best disease (in whom the EOG light rise was abolished); a 54 year old patient with unilateral complete loss of retinal ganglion cells due to a prior ischaemic insult; a 36 year old patient with Oguchi disease secondary to bi-allelic variants in GRK1; a 69 year old patient with a unilateral inflammatory retinopathy in whom rod-driven bipolar cell signals were absent. All of the patients showed similar kinetics, except for the patient with GRK1-associated Oguchi disease, in whom the increase was less than $15 \%$.

Conclusion In healthy subjects, response amplitudes increase significantly over $10 \mathrm{~min}$ (the standard ISCEV light adaptation period). This increase does not appear to depend on change in voltage over the RPE that occurs over a comparable time course, nor is it dependent on ganglion cell input (despite animal studies suggesting a role for intrinsically photosensitive ganglion cells in ERG light adaptation). However, continual suppression of the rod circulating current in the dark (and consequent rod hyperpolarisation, as occurs in Oguchi disease) appears to largely diminish the subsequent Light Adaptation Effect, implicating a drive from rod signalling. The normal Light Adaptation Effect in the patient with absent rod-driven bipolar cell responses suggests that this input might occur earlier in the visual pathway, possibly via gap junctions between rod and cone photoreceptors.

Conflict of interest: None

Funding: Wellcome Trust; Moorfields Eye Charity Award applications: None.

Corresponding author email: o.mahroo@ucl.ac.uk

\section{Oral Session 4: Genotype-Phenotype Correlations}

O4.1 Spectrum of occult macular dysfunction syndrome (OMDS): investigation of RP1L1-positive occult macular dystrophy and RP1L1-negative occult macular dystrophys; EAOMD report 5

Yang, Lizhu ${ }^{1,2,3}$, Joo, Kwangsic ${ }^{4}$, Tsunoda, Kazushige ${ }^{1}$, Fujinami-Yokokawa, Yu ${ }^{1,5,6,7}$, Pontikos, Nikolas ${ }^{1,8,9}$, Arno, Gavin ${ }^{1,8,9}$, Kondo, Mineo ${ }^{10}$, Liu, Xiao ${ }^{1,2,11}$ ,Kurihara, Toshihide ${ }^{2}$, Tsubota, Kazuo ${ }^{2}$, Yoshitake, Kazutoshi $^{12}$, Iwata, Takeshi ${ }^{12}$, Yamazawa, Kazuki ${ }^{13,14}$, Zou, Xuan ${ }^{3}$, Li, Hui ${ }^{3}$, Park, Kyu Hyung ${ }^{4}$, Kim, Min Seok ${ }^{4}$, Mun, Yong Seok ${ }^{4}$, Robson, Anthony G. ${ }^{8,9}$, Miyake, Yozo ${ }^{1,15,16}$, Woo, Se Joon ${ }^{4}$, Sui, Ruifang ${ }^{3}$, Fujinami, Kaoru $^{1,2,8,9}$, East Asia Inherited Retinal Disease Society ${ }^{17}$

${ }^{1}$ Laboratory of Visual Physiology, Division of Vision Research, National Institute of Sensory Organs, National Hospital Organization, Tokyo Medical Center, Tokyo, Japan., ${ }^{2}$ Department of Ophthalmology, Keio University School of Medicine, Tokyo, Japan., ${ }^{3}$ Department of Ophthalmology, Peking Union Medical College Hospital, Peking Union Medical College and Chinese Academy of Medical Sciences, Beijing, China., ${ }^{4}$ Department of Ophthalmology, Seoul National University Bundang Hospital, Seoul National University College of Medicine, Seongnam, South Korea., ${ }^{5}$ Department of Health Policy and Management, Keio University School of Medicine, Tokyo, Japan, ${ }^{6}$ Department of Public Health Research, Yokokawa clinic, Osaka, Japan., ${ }^{7}$ UCL Institute of Ophthalmology, London, UK, ${ }^{8} \mathrm{UCL}$ Institute of Ophthalmology, London, UK., ${ }^{9}$ Moorfields Eye Hospital, London, UK, ${ }^{10}$ Department of Ophthalmology, Mie University Graduate School of Medicine, Tsu, Mie, Japan., ${ }^{11}$ Army Medical University, Southwest Hospital, Chongqing, China., ${ }^{12}$ Division of Molecular and Cellular Biology, National Institute of Sensory Organs, National Hospital Organization Tokyo Medical Center, Tokyo, Japan., ${ }^{13}$ Department of Pediatrics, National Hospital Organization Tokyo Medical Center, Tokyo, Japan., ${ }^{14}$ Medical Genetics Center, National Hospital Organization Tokyo Medical Center, Tokyo, Japan., ${ }^{15}$ Aichi Medical University, Nagakute, Aichi, Japan., ${ }^{16}$ Next Vision, Kobe Eye Center, Kobe, Hyogo, Japan., ${ }^{17}$ EAIRDs 
Purpose Occult Macular Dysfunction Syndrome (OMDS) is characterized by a normal funduscopic appearance and macular dysfunction. This study compares the clinical features between RP1L1-positive occult macular dystrophy (RP1L1-positive Occult Macular Dysfunction; Miyake disease) and RP1L1negative cases in an expanded East Asian cohort.

Methods Thirty-six affected subjects from 21 families with RP1L1-positive Occult Macular Dysfunction (group A) and 31 subjects from 27 families with RP1L1-negative Occult Macular Dysfunction (group B) were enrolled from the database of the East Asia Inherited Retinal Disease Society. The examined eyes were classified into one of two groups based on the results of spectral-domain optical coherence tomography (OCT; Fujinami K, Yang L, Joo K et al., Ophthalmol 2019; 126:1432-1444); (i) classical change with blurred ellipsoid zone (EZ) and absence of interdigitation zone (IZ) or (ii) subtle change (minimum/local blurring of EZ and local absence of IZ). The subjects were also classified into one of three functional phenotypes based on mfERG findings (Yang et al. ISCEV 2017, 2019); group 1-paracentral dysfunction, group 2-homogeneous central dysfunction, and group 3-widespread dysfunction.

Results There were 29 families (66.7\%) with autosomal dominant (AD) family history (FH) and seven subjects $(33.3 \%)$ with unknown FH in group A; eight AD families (29.6\%), two autosomal recessive families $(7.4 \%)$ and 17 (63.0\%) subjects with unknown $\mathrm{FH}$ in group $\mathrm{B}$. The median visual acuity (VA) was $0.65(-0.08-1.22) / 0.52(-0.18-1.4)$ in the $\log$ MAR unit in group $\mathrm{A} / \mathrm{B}(p=0.006)$. OCT demonstrated classical changes in $60(83.3 \%) / 17(28.3 \%)$ eyes of group A/B; subtle changes in $12(16.7 \%) / 22(36.7 \%)$ eyes of group A/B, while $21(35.0 \%)$ eyes in group B showed other OCT changes $(p<0.001)$. The mfERGs revealed a group 1 functional phenotype in eight $(8 /$ $53,15.1 \%) /$ three $(3 / 40,7.5 \%)$ eyes of group $\mathrm{A} / \mathrm{B}$; the group 2 functional phenotype occurred in $40(75.5 \%) / 31(77.5 \%)$ eyes of group $\mathrm{A} / \mathrm{B}$; the group 3 functional phenotype was seen in five $(9.4 \%) /$ six $(15.0 \%)$ of group A/B. Four heterozygous disease-causing variants in four genes were identified in five unrelated subjects in group B; GUCY2D (c.2513G $>$ A (p.Arg838His); two subjects), CRX (c.128G $>$ A (p.Arg43His), ATXN1 (43-48 CAG repeats), and ATXN7 (39 CAG repeats).

Conclusion This study further expands the phenotypic and genotypic spectrum of Occult Macular Dysfunction Syndrome and highlights differences between RP1L1-positive and RP1L1-negative cases. RP1L1-positive Occult Macular Dysfunction is associated with a high incidence of outer retinal changes (classical changes). In RP1L1-negative Occult Macular Dysfunction Syndrome there is often milder disruption of the outer retina with a tendency for better preservation of VA. Other genes also related to the clinical presentation of Occult Macular Dysfunction Syndrome include: GUCY2D (Liu X, Fujinami K, et al. Transl Vis Sci Technol. 2020; 9 (6), Article 2), CRX (Fujinami Y, Fujinami K, et al. Sci Rep 2020; 10: Article 9531), ATXN1, and ATXN7 (Park JY et al. Ophthalmic Genet. 2019;40(3):282-285).

Conflict of interest: All authors have completed and submitted the ICMJE Form for Disclosure of Potential Conflicts of Interest. Individual investigators who participate in the sponsored project(s) are not directly compensated by the sponsor but may receive salary or other support from the institution to support their effort on the project(s). Kaoru Fujinami is a paid consultant of Astellas Pharma Inc, Kubota Pharmaceutical Holdings Co., Ltd, Acucela Inc., Novartis AG., Janssen Pharm., Sanofi Genzyme., and NightStar. Kaoru Fujinami reports personal fees from Astellas Pharma Inc, personal fees from Kubota Pharmaceutical Holdings Co., Ltd., personal fees from Acucela Inc., personal fees from SANTEN Company Limited, personal fees from Foundation Fighting Blindness, personal fees from Foundation Fighting Blindness Clinical Research Institute, personal fees from Japanese Ophthalmology Society, personal fees from Japan Retinitis Pigmentosa Society. Laboratory of Visual Physiology, Division for Vision Research, National Institute of Sensory Organs, National Hospital Organization, Tokyo Medical Center, Tokyo, Japan is supported by grants from Astellas Pharma Inc (NCT03281005), outside the submitted work. Se Joon Woo is a paid consultant of Samsung Bioepis Inc., Panolos Bioscience Inc., Novelty Nobility Inc., and a co-founder of Retimark Inc. South Korea.

Funding: Kaoru Fujinami is supported by grants from Grantin-Aid for Young Scientists (A) of the Ministry of Education, Culture, Sports, Science and Technology, Japan (16H06269), Grant-in-Aid for Scientists to support international collaborative studies of the Ministry of Education, Culture, Sports, Science and Technology, Japan (16KK01930002), National Hospital Organization Network Research Fund, Japan (H30NHO-Sensory Organs-03), AMED (18ek0109355h0001), Foundation Fighting Blindness Alan Laties Career Development Program (CF-CL-0416-0696-UCL), USA; Health Labour Sciences Research Grant, The Ministry of Health Labour and Welfare, Japan (201711107A), Great Britain Sasakawa Foundation Butterfield Awards, UK. Kazushige Tsunoda is supported by Japan Agency for Medical Research and Development, the Ministry of Health, Labor and Welfare, Japan (18ek0109282h0002); Grants-for Scientific Research, Japan Society for the Promotion of Science, Japan (H26-26462674). Yu Fujinami-Yokokawa is supported by grants from Grant-inAid for Young Scientists of the Ministry of Education, Culture, Sports, Science and Technology, Japan (18K16943). Gavin Arno is supported by Fight for Sight (UK) Early Career Investigator award, NIHR-BRC at Moorfields Eye Hospital and the UCL Institute of Ophthalmology, and Great Britain Sasakawa Foundation Butterfield Award, UK. Anthony G. Robson is supported by NIHR Biomedical Research Centre at Moorfields Eye Hospital NHS Foundation Trust and UCL Institute of Ophthalmology and by the Moorfields Eye Charity. Toshihide Kurihara is supported by Tsubota Laboratory, Inc, Fuji Xerox Co, Ltd, Kirin Company, Ltd, Kowa Company, Ltd, Novartis Pharmaceuticals, Santen Pharmaceutical Co, Ltd, ROHTO Pharmaceutical Co, Ltd. Takeshi Iwata is supported by AMED, Japan; the Ministry of Health, Labor and Welfare, Japan (18ek0109282h0002), Grants-in-Aid for Scientific Research, Japan; and National Hospital Organization Network Research Fund (H30-NHO-Sensory Organs-02). Se Joon Woo is supported by a research grant from the Seoul National University Bundang Hospital (16-2019-003), National Research Foundation of Korea grant 2016R1D1A1B03934724, funded by the Korean government (Ministry of Science, ICT and Future Planning [MSIP]). Ruifang Sui is supported by grants from Foundation Fighting Blindness (CD-CL-0214-0631-PUMCH); CAMS Innovation 
Fund for Medical Sciences, China, CIFMS 2016-12M-1-002; National Natural Science Foundation of China, China, 81470669. Role of the Funder/Sponsor: The funding sources had no role in the design and conduct of the study; collection, management, analysis, and interpretation of the data; preparation, review, or approval of the manuscript; and decision to submit the manuscript for publication.

Award applications: None.

Corresponding author email: k.fujinami@ucl.ac.uk

O4.2 The clinical and genetic spectrum of conedominated retinal dystrophies with normal funduscopic appearance

Fujinami, Kaoru ${ }^{1,2,3,4}$, Ueno, Shinji ${ }^{5}$, Hayashi, Takaaki ${ }^{6}$, Kuniyoshi, Kazuki ${ }^{7}$, Kondo, Mineo ${ }^{8}$, Mizota, Atsushi', Shinoda, Kei ${ }^{9,10}$, Mochizuki, Kiyofumi ${ }^{11}$, Kameya, Shuhei $^{12}$, Ideta, Ryuichi ${ }^{13}$, Naoi, Nobuhisa ${ }^{14}$, Ikeda, Yasuhiro $^{14}$, Fujinami-Yokokawa, Yu ${ }^{1,3,15,16}$, Yang, Lizhu $^{1,2}$, Liu, Xiao ${ }^{1,2,17}$, Arno, Gavin ${ }^{1,3,18,19}$, Pontikos, Nikolas $^{1,3,18}$, Kominami, Taro ${ }^{5}$, Katagiri, Satoshi ${ }^{6}$, Mizobuchi, Kei ${ }^{6}$, Sakuramoto, Hiroyuki ${ }^{7}$, Nakamura, Natsuko ${ }^{1,9,20}$, Kikuchi, Sachiko ${ }^{12}$, Mawatari, Go ${ }^{21}$, Kurihara, Toshihide $^{2}$, Tsubota, Kazuo ${ }^{2}$, Terasaki, Hiroko $^{5}$, Yoshitake, Kazutoshi ${ }^{22}$, Miyake, Yozo ${ }^{1,23,24}$, Iwata, Takeshi $^{22}$, Tsunoda, Kazushige ${ }^{1}$, Japan Eye Genetics Consortium study group ${ }^{1}$

${ }^{1}$ Laboratory of Visual Physiology, Division of Vision Research, National Institute of Sensory Organs, National Hospital Organization, Tokyo Medical Center, Tokyo, Japan., ${ }^{2}$ Department of Ophthalmology, Keio University School of Medicine, Tokyo, Japan., ${ }^{3}$ UCL Institute of Ophthalmology, London, UK., ${ }^{4}$ Moorfields Eye Hospital, London, UK., ${ }^{5}$ Department of Ophthalmology, Nagoya University Graduate School of Medicine, Nagoya, 466-8550, Japan., ${ }^{6}$ Department of Ophthalmology, The Jikei University School of Medicine, Tokyo, 105-8461, Japan., ${ }^{7}$ Department of Ophthalmology, Kindai University Faculty of Medicine, Osaka-Sayama, 589-8511, Japan., ${ }^{8}$ Department of Ophthalmology, Mie University Graduate School of Medicine, Mie, Japan, ${ }^{9}$ Department of Ophthalmology, Teikyo University, Tokyo, 173-8605, Japan., ${ }^{10}$ Department of Ophthalmology, Saitama Medical University, Saitama, 350-0495, Japan., ${ }^{11}$ Department of Ophthalmology, Gifu University Graduate School of Medicine, 1-1 Yanagido, Gifu-shi, Gifu, 501-1194 Japan, ${ }^{12}$ Department of Ophthalmology, Nippon Medical School Chiba Hokusoh Hospital, Inzai, 270-1694, Japan., ${ }^{13}$ IDETA Eye Hospital, Kumamoto City, Kumamoto, Japan, ${ }^{14}$ Department of Ophthalmology, University of Miyazaki, Miyazaki, 889-2192, Japan, ${ }^{15}$ Department of Health Policy and Management, Keio University School of Medicine, Tokyo, Japan., ${ }^{16}$ Division of Public Health, Yokokawa Clinic, Suita, 564-0083, Japan., ${ }^{17}$ Southwest Hospital/ Southwest Eye Hospital, Third Military Medical University (Army Medical University), Chongqing, China.,

${ }^{18}$ Moorfields Eye Hospital, London, UK, ${ }^{19}$ North East Thames Regional Genetics Service, UCL Great Ormond Street Institute of Child Health, Great Ormond Street NHS
Foundation Trust, London, WC1N 1EH, UK., ${ }^{20}$ Department of Ophthalmology, The University of Tokyo, Tokyo, 113-8654, Japan., ${ }^{21}$ Department of Ophthalmology, University of Miyazaki, Miyazaki, 889-2192, Japan., ${ }^{22}$ Division of Molecular and Cellular Biology, National Institute of Sensory Organs, National Hospital Organization Tokyo Medical Center, Tokyo, 152-8902, Japan., ${ }^{23}$ Aichi Medical University, Nagakute, Aichi, 480-1195, Japan.,

${ }^{24}$ Next Vision, Kobe Eye Center, Hyogo, 650-0047, Japan

Purpose There are several cone-dominated dystrophies (CDD) that show normal funduscopic appearance; however, the prevalence of each causative gene and the phenotypic overlap caused by each gene within the CDD are still uncertain. We herein illustrate the clinical and genetic spectrum of conedominated retinal dystrophies with normal funduscopic appearance (CDD-NF) in a nationwide cohort.

Methods 1302 patients with available genotype-phenotype correlation analysis results registered in the database of the Japan Eye Genetic Consortium (JEGC) from 2008 to 2018 were surveyed. Patients were classified into one of the three disease categories based on the electrophysiological findings of full-field ERGs, mfERGs, and focal macular ERGs: occult macular dysfunction syndrome (OMDS: confined macular dysfunction, including hereditary occult macular dystrophy (OMD) and occult maculopathy); cone dystrophy with normal funduscopic appearance (CORD-NF: progressive generalized cone (-rod) dysfunction); and cone dysfunction syndrome (CDS: non-progressive generalized cone dysfunction). The clinical parameters were compared among the three disease categories, and the overlap of causative genes was investigated in genes identified in multiple families.

Results 192 patients from 150 families with CDD-NS were recruited, including 76 families with OMDS, 43 families with CORD-NF, and 31 families with CDS. The median age of onset was 30.0 years (range, 3-70) for OMD, 30.0 years (range, 3-75) for CORD-NF, and 0.0 years (range, 0-56) for $\mathrm{CDS}$, respectively. The median visual acuity (VA) in the logarithm of the minimum angle of resolution ( $\log M A R)$ unit was 0.52 (range, 0-1.22) for OMD, 0.70 (range, -0.18-2.0) for CORD-NF, and $0.80(0-1.70)$, respectively. The causative genes were RP1L1, GUCY2D, and CRX for OMD, POC1B, GUCY2D, CRX, PDE6C, and CRB1 for CORD-NF, and CNGA3, PDE6C, GNAT2, KCNV2, and RGS9BP for CDS. Out of eight genes identified in multiple families, four genes were found only in a single disease category: RP1L1 (OMD; Miyake disease), CNGA3 (CDS), GNAT2 (CDS), and KCNV2 (CDS); while four genes overlapped the disease categories; POC1B (CORD-NF/CDS), PDE6C(CORD-NF/CDS), GUCY2D (CORD-NF/OMD), and CRX (CORD-NF/OMD).

Conclusion The clinical spectrum of CDD-NF was first identified in a large well-characterized cohort: OMD with later-onset and moderate VA decrease, CORD-NF with lateronset and severe VA impairment, and CDS with early-onset and more severe VA decline. The heterogeneous genetic background of CDD-NF was revealed; meanwhile, distinct genotype-phenotype relations such as RP1L1-OMD, CNGA3$\mathrm{CDS}$, and GNAT2-CDS were confirmed, given KCNV2 typically presents visible macular atrophy.

Conflict of interest: All authors have completed Disclosure of Potential Conflicts of Interest. Individual investigators who 
participate in the sponsored project(s) are not directly compensated by the sponsor but may receive salary or other support from the institution to support their effort on the project(s). Kaoru Fujinami is a paid consultant of Astellas Pharma Inc, Kubota Pharmaceutical Holdings Co., Ltd, and Acucela Inc. and NightstaRx Limited. Kaoru Fujinami reports personal fees from Astellas Pharma Inc, personal fees from Kubota Pharmaceutical Holdings Co., Ltd., personal fees from Acucela Inc., personal fees from NightstaRx Limited., personal fees from SANTEN Company Limited, personal fees from Foundation Fighting Blindness, personal fees from Foundation Fighting Blindness Clinical Research Institute, personal fees from Japanese Ophthalmology Society, personal fees from Japan Retinitis Pigmentosa Society. Laboratory of Visual Physiology, Division for Vision Research, National Institute of Sensory Organs, National Hospital Organization, Tokyo Medical Center, Tokyo, Japan is supported by grants from Astellas Pharma Inc (NCT03281005), outside the submitted work.

Funding: Kaoru Fujinami is supported by grants from National Hospital Organization Network Research Fund (H30-NHOSensory Organs-03), grants from FOUNDATION FIGHTING BLINDNESS ALAN LATIES CAREER DEVELOPMENT PROGRAM (CF-CL-0416-0696-UCL), grants from Health Labour Sciences Research Grant, The Ministry of Health Labour and Welfare (201711107A), and grants from Great Britain Sasakawa Foundation Butterfield Awards. Yu Fujinami-Yokokawa was supported by grants from Grant-in-Aid for Young Scientists of the Ministry of Education, Culture, Sports, Science and Technology, Japan (18K16943). Toshihide Kurihara is supported by Tsubota Laboratory, Inc, Fuji Xerox Co., Ltd, Kirin Company, Ltd, Kowa Company, Ltd, Novartis Pharmaceuticals, Santen Pharmaceutical Co. Ltd, and ROHTO Pharmaceutical Co.,Ltd, outside the submitted work Role of the Funder/Sponsor: The funding sources had no role in the design and conduct of the study; collection, management, analysis, and interpretation of the data; preparation, review, or approval of the manuscript; and decision to submit the manuscript for publication.

Award applications: None.

Corresponding author email: k.fujinami@ucl.ac.uk

\section{O4.3 Phenotypic characterization of Stargardt disease cases carrying the frequent $\mathrm{ABCA} 4$ mutation c.5714 + 5G > A (p.[= ,Glu1863Leufs*33]) using electrophysiology}

Sajovic, Jana ${ }^{1}$, Meglič, Andrej ${ }^{1}$, Šuštar, Maja ${ }^{1}$, JarcVidar, Martina ${ }^{1}$, Cremers, Frans ${ }^{2,3}$, Khan, Mubeen ${ }^{2,3}$, Allikmets, Rando ${ }^{4,5}$, Glavač, Damjan ${ }^{6}$, Maver, Aleš ${ }^{7}$, Volk, Marija $^{7}$, Peterlin, Borut ${ }^{7}$, Hawlina, Marko ${ }^{1}$, Fakin, Ana ${ }^{1}$

${ }^{1}$ Eye Hospital, University Medical Centre Ljubljana, Ljubljana, Slovenia, ${ }^{2}$ Department of Human Genetics, Radboud University Medical Center, Nijmegen, The Netherlands, ${ }^{3}$ Donders Institute for Brain, Cognition and Behaviour, Radboud University Medical Center, Nijmegen, The Netherlands, ${ }^{4}$ Department of Ophthalmology, Columbia University, New York, USA, ${ }^{5}$ Department of Pathology \& Cell Biology, Columbia University, New
York, USA, ${ }^{6}$ Department of Molecular Genetics, Institute of Pathology, Faculty of Medicine, University of Ljubljana, Ljubljana, Slovenia, ${ }^{7}$ Clinical Institute for Medical Genetics, University Medical Centre Ljubljana, Ljubljana, Slovenia

Purpose To describe the isolated effect of ABCA4 mutation c.5714 + 5G > A (p.[=, Glu1863Leufs*33]) on retinal structure and function.

Methods The study included 15 patients with ABCA4 retinopathy (Stargardt disease) who were divided into two groups based on their genotype. Patients in group 1 harboured the splicing mutation c.5714 + 5G > A (p. [=, Glu1863Leufs*33]) in trans with a null mutation, whereas patients in group 2 (control group) had double null mutations. Group 1 consisted of seven patients (4 male, median age 21 years, range 12-55) and group 2 of eight patients ( 2 male, median age 21 years, range 8-37). Age at the time of exam, age at onset and decimal Snellen visual acuity were collected from the medical records. Patients' right eyes were taken for the analysis. Areas of definitely decreased autofluorescence (DDAF) within the $30^{\circ} \times 30^{\circ}$ fundus autofluorescence images were measured using custom-made software detecting DDAF based on ProgStar criteria (1). Pattern electroretinograms (PERG) to $24^{\circ} \times 30^{\circ}$ (large field) checkerboard and full-field electroretinography (dark-adapted 3 ERG and light-adapted $30 \mathrm{~Hz}$ flicker ERG) were recorded according to ISCEV standards. Correlations between structure (DDAF) and function (PERG, ffERG), according to the genotype, were analysed using multiple linear regression.

Results Group 1 had significantly later median age of onset than group 2 (17 vs 8 years; $p<0.01$ ) and significantly better visual acuity ( 0.4 vs $0.1 ; p<0.01$; Mann-Whitney U Test). Median PERG P50 amplitudes for groups 1 and 2 were $3.1 \mu \mathrm{V}$ (range $0.0-5.5$ ) and $0.5 \mu \mathrm{V}$ (range 0.0-1.4). Median DA 3 ERG a-wave amplitude for group 1 was $132 \mu \mathrm{V}$ (range 26-213) and for group 2, $47 \mu \mathrm{V}$ (range 0-109). Median LA $30 \mathrm{~Hz}$ flicker ERG amplitude for group 1 was $49 \mu \mathrm{V}$ (range 9-137) and for group 2, $10 \mu \mathrm{V}$ (range 0-26). After adjusting for age, group 1 had significantly higher PERG P50, DA 3 ERG a-wave and LA $30 \mathrm{~Hz}$ flicker ERG amplitudes and significantly smaller areas of DDAF (multiple linear regression; $p<0.01$ for all). Multiple linear regression showed significant correlation between PERG P50 and DDAF within each genotype group; where patients in group 1 had higher P50 amplitudes than group 2 patients with similarly sized DDAF areas $(p<0.01)$.

Conclusion Electrophysiology can be used to quantitatively assess differences in the function of the retina in different Stargardt genotypes. Patients with the splicing mutation c.5714 + 5G > A (p.[=, Glu1863Leufs*33]) displayed significantly milder phenotypes than double null patients for all parameters. Furthermore, they had higher PERG P50 amplitudes that were not expected according to the DDAF areas of similar sizes. This suggests relative photoreceptor sparing produced by the c.5714 $+5 \mathrm{G}>\mathrm{A}$ allele, while double null patients displayed early photoreceptor dysfunction.

1. Strauss RW et al. JAMA Ophthalmol. 2019, 137:1134-1145.

Conflict of interest: None.

Funding: J3-1750 ARRS.

Award applications: None.

Corresponding author email: ana.fakin@gmail.com 
O4.4 The phenotypic variability and age-related electrophysiological features of Enhanced S-cone syndrome in a series of 56 patients

Robson, Anthony $\mathbf{G}^{1,2}$, De Carvalho, Emanuel ${ }^{1,2}$, Arno, Gavin $^{1,2}$, Boon, Camiel ${ }^{3,4}$, Webster, Andrew R $\mathbf{R}^{1,2}$, Michaelides, Michel ${ }^{1,2}$

${ }^{1}$ Moorfields Eye Hospital, London, UK; ${ }^{2}$ UCL Institute of Ophthalmology, London, UK; ${ }^{3}$ Ophthalmology,

Amsterdam University Medical Centers, Amsterdam, the Netherlands; ${ }^{4}$ Ophthalmology, Leiden University Medical Center, Leiden, the Netherlands

Purpose To describe the clinical and age-related electrophysiological features in patients with Enhanced S-Cone Syndrome (ESCS).

Methods The clinical findings in 56 patients (age range 1-75 years) diagnosed with ESCS were reviewed, including 30 that had undergone ISCEV Standard pattern and full-field electroretinography (PERG; ERG). Diagnosis was established by molecular confirmation of disease-causing variants in the NR2E3 gene $(n=38)$ or by pathognomonic ERG features.

Results The mean age of onset of symptoms was 4 years, and median age at presentation was 20 years. Nyctalopia was the most common initial symptom, reported in 52 patients. Clinical findings included macular schisis $(n=26)$, yellow/white dots $(\mathrm{n}=32)$, nummular pigmentation $(\mathrm{n}=48)$, torpedo-like lesions $(n=6)$ and circumferential subretinal fibrosis $(n=4$ patients). The ERGs in one patient were undetectable; others had pathognomonic ERG features. In those with detectable responses, mean amplitudes of the DA 10.0 ERG a- and b-waves were reduced by $52 \%$ and $63 \%$, respectively and mean a- and b-wave peak times were $16 \mathrm{~ms}$ and $14 \mathrm{~ms}$ longer, respectively compared with those for the control group. The mean amplitudes of the LA $30 \mathrm{~Hz}$ ERG and the LA 3.0 ERG b-waves were reduced by $93 \%$ and $69 \%$ respectively and mean peak times were $14 \mathrm{~ms}$ and $20 \mathrm{~ms}$ longer, respectively, compared with those for the control group. The mean amplitude ratio between the LA 3.0 ERG a-wave and LA $30 \mathrm{~Hz}$ ERG was $1.86(\mathrm{SD}=0.9)$ in the ESCS cohort and 0.37 $(\mathrm{SD}=0.09)$ in healthy controls. There was evidence of age associated ERG reduction in DA and LA ERGs at a rate that was indistinguishable from that seen in controls over a range of more than 6 decades. There was significant negative correlation between age and PERG P50 amplitude $(\mathrm{R}=-0.65, p<0.05)$. Conclusion ESCS has a highly variable clinical phenotype. The ERGs have pathognomonic waveform features in most but with marked amplitude variability. The mean rate of agerelated decline is indistinguishable from control subjects, consistent with largely stable peripheral retinal dysfunction. Pattern ERGs are consistent with progressive macular dysfunction.

Conflict of interest: None.

Funding: NIHR Biomedical Research Centre at Moorfields Eye Hospital and the UCL Institute of Ophthalmology \& The Moorfields Eye Charity.

Award applications: None.

Corresponding author email: anthony.robson3@nhs.net
O4.5 Clinical and electrophysiological characterization of patients with retinal dysfunction consequent upon variants of CACNA1F and CABP4

Ba-Abbad, Rola ${ }^{1,2}$, Calcangi, Antonio ${ }^{1}$, Arno, Gavin ${ }^{1,3}$, Vincent, Ajoy ${ }^{4}$, Locke, Jeff ${ }^{4}$, Mahroo, Omar A., Michaelides, Michel ${ }^{1,5}$, Webster, Andrew R. ${ }^{1,5}$, Robson, Anthony G. ${ }^{1,5}$

${ }^{1}$ Moorfields Eye Hospital, London, UK; ${ }^{2}$ King Khaled Eye Specialist Hospital, Riyadh, Saudi Arabia; ${ }^{3}$ UCL Institute of Ophthalmology, University College London, London, UK; ${ }^{4}$ Hospital for Sick Children, Toronto, Canada

Purpose To describe and compare the clinical and electrophysiological features of patients with "incomplete congenital stationary night blindness" due to variants in the CACNA1F and CABP4 genes.

Methods The medical records of patients with diseaseassociated variants in $\mathrm{CACNA} 1 \mathrm{~F}$ and $\mathrm{CABP} 4$ were reviewed at the inherited retinal disorders clinics at Moorfields Eye Hospital in London and at the Hospital for Sick Children in Toronto. Data analyzed included medical history, best corrected visual acuity, fundus digital imaging, and optical coherence tomography (OCT). ISCEV Standard full-field electroretinography (ERG) data recorded with gold foil corneal electrodes were available in 27 cases.

Results Patients with X-linked CACNA1F-retinopathy $(n=33)$ had subnormal visual acuity, with or without nystagmus, and four patients noted reduced vision in the dark. Patients with autosomal recessive CABP4-retinopathy $(n=9)$ had severely reduced visual acuity (20/200 or worse), nystagmus, and photophobia. Scotopic strong flash ERGs were abnormal in all cases, with DA10 ERG b:a ratios ranging from 0.3 to 1.1 (CACNA1F; $\mathrm{n}=22$ ) and from 0.4 to 0.7 (CABP4; $\mathrm{n}=5$ ). Photopic ERGs were abnormal in all cases including one patient in the CABP4 group with undetectable LA $30 \mathrm{~Hz}$ flicker and LA 3.0 responses. Retinal imaging showed foveal hypoplasia in three patients with CACNA1F retinopathy and ellipsoid zone (EZ) defects of variable severity in seven patients with CABP4 retinopathy.

Conclusion Patients with retinal dysfunction due to variants in CACNA1F and CABP4 show an overlapping phenotypic spectrum consistent with a synaptic disorder of rods and cones. CABP4-related disease is associated with worse visual acuity and may cause more severe cone system dysfunction than that in CACNA1F-retinopathy. Foveal hypoplasia in patients with CACNA1F-retinopathy and an optical gap of the EZ on OCT in CABP4-related cases suggest a role for both proteins in foveal development.

Conflict of interest: None

Funding: None.

Award applications: None.

Corresponding author email: anthony.robson3@nhs.net 
O4.6 DNA testing for inherited retinal disease: Initial experience with the SPARK/Invitae 248-gene panel

Brodie, Scott, Dedania, Vaidehi

Department of Ophthalmology, NYU Langone Health, New York, USA

Purpose To evaluate the diagnostic yield and clinical impact of the SPARK/Invitae 248 gene panel in patients with known or suspected inherited degenerative retinal disease, in comparison with traditional clinical assessments including ERG testing.

Methods Patients were drawn from the clinical practices of the authors. Genetic screening was provided by means of the SPARK/Invitae 248-gene panel at no charge to patients. Over the past year, results are available for 18 patients. Clinical diagnoses were Best vitelliform dystrophy (definite in one patient, suspected in three patients); retinitis pigmentosa (five affected patients, one likely female carrier); possible Stargardt disease (five patients); and one each with Leber congenital amaurosis, choroideremia, nyctalopia, and Norrie disease.

Results Of the 18 studies returned, results were deemed by Invitae "Positive" in nine and "Uncertain" in nine. The genetic diagnosis agreed with the clinical diagnosis in eight cases (including two patients diagnosed with retinitis pigmentosa with homozygous mutations in USH2A, and one patient with the diagnosis of retinitis pigmentosa with compound heterozygotic mutations in NR2E3, which is also associated with enhanced S-cone syndrome). One patient with macular degeneration of uncertain etiology had a deletion in TPP1, which codes for ceroid lipofuscinosis. The remaining nine patients all returned heterozygous mutations considered "variations of uncertain significance" (VUS) ranging from two to eight different genes. Heterozygous VUS in ABCA4 were noted in two patients with the clinical diagnosis of Stargardt disease and in one with the clinical diagnosis of Best disease. Conclusion The SPARK/Invitae 248-gene panel provided a genetic diagnosis consistent with the clinical diagnosis in about half of cases and was diagnostic in one case where the clinical diagnosis was unclear. Retinitis pigmentosa and Stargardt disease were the most common genetic diagnoses. No cases of Leber congenital amaurosis were detected by genetic testing which had been misdiagnosed clinically.

Conflict of interest: None.

Funding: None.

Award applications: None.

Corresponding author email: scott.brodie@nyumc.org

\section{Oral Session 5: Exploring Novel Strategies}

\section{O5.1 Portable device for VEP examination: continued development}

Kuba, Miroslav, Kremláček, Jan, Vít, František, Kubová, Zuzana, Langrová, Jana, Szanyi, Jana

Electrophysiological Lab, Charles University - Faculty of Medicine, Hradec Králové, Czech Republic

\section{Purpose}

Two years ago at the ISCEV meeting in Reims, we demonstrated a new portable device for examination of VEPs for which we received the Marmor Award for Clinical Innovation (initial award). In the meantime, we have tested practical use of the device and, on the basis of the results, some modifications were made.

Methods The main improvement represents introduction of an additional external visual stimulator. The primary stimulator, based on a matrix of 32 LEDs built into the front of the device (distance from eyes is $7.5 \mathrm{~cm}$ ), did not provide sufficiently high spatial frequency of pattern-reversal stimulation, and recorded VEPs had lower sensitivity in detection of the optic pathway pathologies (e.g. in optic neuritis, multiple sclerosis). The newly modified device can be connected and synchronized with a tablet generating standard pattern-reversal stimuli (check sizes $60^{\prime}$ and $15^{\prime}$ according to ISCEV standards) and any other stimuli, e.g. radial motion of a low contrast pattern for evaluation of motion-onset VEPs.

Results In 70 healthy subjects, it was verified that the parameters of VEPs recorded with the use of the new portable device did not differ significantly from parameters of VEPs acquired in standard laboratory conditions and that their intra- and inter-individual variability was comparable. In 30 patients with optic neuritis (multiple sclerosis), the sensitivity of VEP examination with the use of the portable device did not differ from standard equipment, and moreover, its use enabled us to examine fully immobile patients even in their sitting or lying position (thanks to a special adjustable holder of the stimulating tablet) at their home environment. In 15 schizophrenic patients, VEP examination was done during their visit in the psychiatric ambulance with the use of cognitive visual stimuli (confirming prolonged P300 latency in the majority of them). It was demonstrated that the device can be easily used even in poorly cooperative patients.

Conclusion We believe that the new version of the device is now ready for its diagnostic applications not only in neuroophthalmology but, owing to its mobility (all parts can be carried in one bag), also in other situations where functions of the visual pathway or CNS should be immediately verified. We intended again to demonstrate the updated prototype live at the ISCEV 2020 meeting, but unfortunately due to the COVID-19 it is not possible this year. A small production run of the device is now under construction and will be available to those who would like to help with further testing. We are currently in search for a manufacturer of the device, the price of which should not exceed approximately $\$ 1,500$ US.

Conflict of interest: None.

Funding: Financially supported by Grant Agency of the Czech Ministry of Health (AZV-NV18-08-00314) and by the programme PROGRES Q40/07.

Award applications: Marmor Award.

Corresponding author email: kuba@lfhk.cuni.cz

\section{O5.2 Visual cortex transcranial random noise stimulation increases the amplitude of pattern-reversal VEPs}

Donkor, Richard, McCulloch, Daphne L., Thompson,
Benjamin

School of Optometry and Vision Science, University of Waterloo, Waterloo, Canada 
Purpose Transcranial random noise stimulation (tRNS) is a neuro-modulation technique involving alternating current with a random electrical oscillation spectrum. tRNS applied to visual or motor cortex increases the spontaneous firing rate of cortical cells and can enhance neuroplasticity for perceptual learning tasks. We tested the hypothesis that visual cortex tRNS would induce an acute increase in the amplitude of pattern-reversal VEPs.

Methods Ten healthy adults (age range 21-37 years, six females) completed active and sham tRNS sessions on different days in random sequence. Active tRNS was also randomized to the left or right occipital cortex. tRNS $(2.0 \mathrm{~mA}$, full range frequency $0.1-640 \mathrm{~Hz}$, current density $0.08 \mathrm{~mA} / \mathrm{cm}$ ) was delivered for $20 \mathrm{~min}$ using a DC-Stimulator Plus (Eldith, NeuroConn GmbH, Germany) through saline-soaked surface sponge electrodes $(5 \times 5 \mathrm{~cm})$ placed at either $\mathrm{O} 1$ or $\mathrm{O} 2$ and centered at $\mathrm{Cz}$. Hemifield stimuli were high contrast reversing checks (16 s of arc check width, mean luminance $50 \mathrm{~cd} / \mathrm{m}^{2}$, $15^{\circ}$ square field) presented monocularly first to right eyes, then to left eyes, before and immediately after tRNS. VEPs were recorded using a 32 channel EEG system with an electrode cap and reported for five occipital channels $(\mathrm{O} 1, \mathrm{Oz}, \mathrm{O} 2, \mathrm{P} 7$ and P8). Peak-to-peak amplitude data for N70-P100 and P100N135 were analyzed.

Results Active tRNS significantly increased VEP amplitudes while sham stimulation had no effect. Significant interactions between Condition (active vs. sham) and Time (pre vs. post) were observed for P7 and P8 channels for both N70-P100 and $\mathrm{P} 100-\mathrm{N} 135$. The right eye viewing condition showed larger VEP amplitudes than the subsequent left eye viewing condition. Lateral occipital electrodes exhibited "paradoxical lateralization" as expected for hemifield pattern-reversal VEPs. Conclusion Increased VEP amplitude following tRNS suggest that the continuous opening and closing of sodium channels during tRNS triggers changes within the neurons which increase visual cortex excitability.

Conflict of interest: None.

Funding: Canadian Optometric Education Fund (COETF); NSERC.

Award applications: None.

Corresponding author email: rdonkor@uwaterloo.ca

\section{O5.3 Electrically evoked responses elicited by transcorneal electrical stimulation in patients with retinitis pigmentosa}

Fujinami-Yokokawa, $Y^{1,2,3,4}$, Suzuki, $Y^{1}$, Yang, $L^{5,6}$, Liu, $\mathbf{X}^{1,6,7}$, Miyata, $\mathbf{H}^{2}$, Farmer, $\mathbf{J}^{\mathbf{8}}$, Shinoda9', Tsunoda, $\mathbf{K}^{\mathbf{1 0}}$, Miyake, $Y^{1,11,12}$, Fujinami, $K^{1,4,6,13}$

\footnotetext{
${ }^{1}$ Laboratory of Visual Physiology, Division of Vision Research, National Institute of Sensory Organs, National Hospital Organization Tokyo Medical Center, Tokyo, Japan., ${ }^{2}$ Department of Health Policy and Management, Keio University School of Medicine, Tokyo, Japan., ${ }^{3}$ Division of Public Health, Yokokawa Clinic, Suita, Japan., ${ }^{4}$ UCL Institute of Ophthalmology, London, UK., ${ }^{5}$ aboratory of Visual Physiology, Division of Vision Research, National Institute of Sensory Organs, National Hospital Organization Tokyo Medical Center, Tokyo, Japan.,
}

${ }^{6}$ Department of Ophthalmology, Keio University School of Medicine, Tokyo, Japan., ${ }^{7}$ Southwest Hospital/Southwest Eye Hospital, Third Military Medical University (Army Medical University), Chongqing, China., ${ }^{8}$ Diagnosys LLC, MA, USA., ${ }^{9}$ Department of Ophthalmology, Saitama Medical University Faculty of Medicine, Iruma-gun, Saitama, Japan., ${ }^{10}$ Division of Vision Research, National Institute of Sensory Organs, National Hospital Organization Tokyo Medical Center, Tokyo, Japan., ${ }^{11}$ Aichi Medical University School of Medicine, Nagakute, Aichi, Japan., ${ }^{12}$ Next Vision, Kobe Eye Center, Hyogo, Japan.,

${ }^{13}$ Moorfields Eye Hospital, London, UK

Purpose Electrically evoked response (EER) elicited by trans corneal electrical stimulation is an objective method to evaluate the visual pathway (Potts, 1968-1970; Miyake, 1980-1984, Takei, 1989-1993). This study eventually aims to validate EER in prediction of the efficacy of advanced treatments for diseases with severe photoreceptor damage. We describe EERs in patients with retinitis pigmentosa (RP) and compare the data with healthy subjects.

Methods Twelve eyes of six patients with RP were enrolled. EERs were recorded after $20 \mathrm{~min}$ of dark adaptation using DTL-Fz stimulation and $\mathrm{Oz}-\mathrm{Pz}$ potential recording at current intensities of $0.5 \mathrm{~mA}, 1 \mathrm{~mA}, 1.5 \mathrm{~mA}$, and $2 \mathrm{~mA}$ [Espion Profile electrophysiology system; Diagnosys LLC, MA, USA). Two ground electrodes for the stimulation and the recording were used and care was taken to ensure low impedance between subject and electrodes: the stimulation ground was $<$ $2 \mathrm{k} \mathrm{ohm}$, the DTL was $<4 \mathrm{k}$ ohm and all other electrodes were $<10 \mathrm{k}$ ohms. The presence of EER responses $(>0.8 \mu \mathrm{V})$ for each component/stimulus condition was evaluated. The sum of EER amplitudes [30-90 ms (P1), 90-150 ms (P2), and 150-210 ms (P3)] was calculated. Ten eyes of five healthy participants were also enrolled. The sum of EER amplitudes in RP patients was compared to that of the healthy subjects.

Results The median age of disease onset/age at examination of six patients was 18.0 (range, 7-42) years/57.0 (38-65) years, respectively. The median visual acuity was $2.0(0.2-2.8) / 2.0$ (0.2-2.8) $\log$ MAR in the right/left eye, respectively. The fullfield ERG responses were undetectable both in the dark- and the light-adapted conditions in the six RP patients. The presence of positive peaks for $0.5 \mathrm{~mA} / 1.0 \mathrm{~mA} / 1.5 \mathrm{~mA} /$ $2.0 \mathrm{~mA}$ components was identified in $41.7 \% / 66.7 \% / 83.3 \%$ / $75 \%$ in the six RP patients. The median value of the sum of EER amplitudes in the six RP patients was 1.75 (range, 0.56-4.86). The presence of positive peaks for $0.5 \mathrm{~mA} /$ $1.0 \mathrm{~mA} / 1.5 \mathrm{~mA} / 2.0 \mathrm{~mA}$ components was identified in $80.0 \%$ / $100 \% / 100 \% / 100 \%$ in the five healthy subjects. The median value of the sum of EER amplitudes in five healthy subjects was 3.61 (range, 1.38-16.48). A statistically significant difference in terms of the sum of EER amplitudes was revealed between the RP patients and the healthy subjects.

Conclusion The presence of positive responses in EERs was more frequently $(>80 \%)$ identified in RP eyes with undetectable ERG responses than seen in a previous report $(5 / 14$, 35.7\%; Miyake 1981). (However, note that the stimulus and recording conditions are different between the two studies.) The sum of EER amplitudes, significantly reduced in the RP patients, can be a parameter to evaluate the visual pathway even when ERGs are undetectable. These findings imply that 
EERs derive predominantly from residual retinal ganglion cell function in RP patients.

Conflict of interest: Jeffrey Farmer is the CEO of Diagnosys LLC. Kaoru Fujinami is a paid consultant of Astellas Pharma Inc, Kubota Pharmaceutical Holdings Co; Ltd, and Acucela Inc. and NightstaRx Limited. Kaoru Fujinami reports personal fees from Astellas Pharma Inc, personal fees from Kubota Pharmaceutical Holdings Co; Ltd; personal fees from Acucela Inc; personal fees from NightstaRx Limited; personal fees from SANTEN Company Limited, personal fees from Foundation Fighting Blindness, personal fees from Foundation Fighting Blindness Clinical Research Institute, personal fees from Japanese Ophthalmology Society, personal fees from Japan Retinitis Pigmentosa Society. Laboratory of Visual Physiology, Division for Vision Research, National Institute of Sensory Organs, National Hospital Organization, Tokyo Medical Center, Tokyo, Japan is supported by grants from Astellas Pharma Inc (NCT03281005), outside the submitted work.

Funding: Yu Fujinami-Yokokawa was supported by grants from Grant-in-Aid for Young Scientists of the Ministry of Education, Culture, Sports, Science and Technology, Japan (18K16943). Kaoru Fujinami is supported by grants from Grant-in-Aid for Young Scientists (A) of the Ministry of Education, Culture, Sports, Science and Technology, Japan (16H06269), grants from Grant-in-Aid for Scientists to support international collaborative studies of the Ministry of Education, Culture, Sports, Science and Technology, Japan (16KK01930002), grants from National Hospital Organization Network Research Fund (H30-NHO-Sensory Organs-03), grants from FOUNDATION FIGHTING BLINDNESS ALAN LATIES CAREER DEVELOPMENT PROGRAM (CF-CL0416-0696-UCL), grants from Health Labour Sciences Research Grant, The Ministry of Health Labour and Welfare (201711107A), and grants from Great Britain Sasakawa Foundation Butterfield Awards.)

Award applications: None.

Corresponding author email: k.fujinami@ucl.ac.uk

\section{O5.4 Acuity VEP: Don't fight over linear vs logarithmic scaling of the spatial frequency axis}

Bach, M

\section{Eye Center, Freiburg University, Germany}

Purpose Objective acuity assessment via the VEP remains a pressing need; any improvement would be welcome. Typically, VEP amplitude (or magnitude) is analysed at various check sizes via their dominant spatial frequency, specifically via a regression to zero, or to noise, or to a $\mu \mathrm{V}$ criterion. It was recently argued (Hamilton et al., Doc Ophthalmol, in press) that this regression should be performed on a linear spatial frequency scale. This implies two separate issues: (1) the spacing of the check sizes (logarithmic or linear), and (2) the scale on which the linear regression is calculated (logarithmic or linear). Problem (1) is overlaid by discreteness issues of pixels - the check size can only span $1 \times 1,2 \times 2,3 \times 3, \ldots$ pixels. We here addressed issue (2).

Methods Available data from Bach et al. (Br J Ophthalmol 2008;92:396-403) were analysed using their "heuristic algorithm" with a linear regression calculated on both linear and logarithmic spatial frequency scaling. The dataset includes 109 observations from 40 normal eyes, 45 normal eyes with artificially reduced acuity and 24 patients. Behavioural visual acuity (VA) ranged from 0.89 to $-0.45 \log$ MAR. BlandAltman "limits of agreement" (LoA) were used to assess prediction of behavioural VA from Acuity VEP.

Results The Acuity VEP results using the heuristic algorithm on a log spatial frequency scale reproduced the behavioural VA within $\pm 0.33595 \%$ CI LoA. Using a linear spatial frequency scale, the LoA increased slightly to \pm 0.363 . This was largely due to more deviation for better acuities $(\leq 0.0 \log$ MAR $)$. The difference in LoA was highly significant $(p<0.0001)$ but only small, $0.028 \log$ MAR. The LoA between the logarithmic or linear extrapolation was much lower, $0.114 \log$ MAR, suggesting little difference between them. Scrutinising all 109 cases revealed some interesting outliers.

Conclusion While there was a slight advantage for log scaling, this may be due to the roughly log-scaled spatial stimulus frequencies used for data acquisition. There was no evidence for a bias with log scaling (as suggested by Hamilton et al.), and the results comply with those by Zhou et al. (Doc Ophthalmol 2008;117:85-91). Since the scaling is generally constrained by pixel size, which leads automatically to a loglike scaling for high spatial frequencies, the difference is somewhat moot and both methods appear roughly equally adequate. We will stick with log scaling.

Conflict of interest: None.

Funding: None.

Award applications: None.

Corresponding author email: michael.bach@uni-freiburg.de

\section{O5.5 Slope between P50 and N95 waves of the pattern electroretinogram (PERG) as an indicator of ganglion cell dysfunction}

\section{Sustar, M, Brecelj, J, Hawlina, M}

Eye Hospital, University Medical Centre Ljubljana, Ljubljana, Slovenia

Purpose In the PERG, reduction of the N95 amplitude and/or decrease of the ratio between the N95 and P50 amplitude commonly indicates a dysfunction at the level of ganglion cells and their axons. By our observations, in cases of such dysfunction, the shape of the N95 wave can be affected in a way that the slope from the top of the P50 towards the N95 is not as steep as in normal subjects. The aim of this study was to explore if the steepness of slope between P50 and N95 waves differed significantly in patients with ganglion cell dysfunction compared to a healthy population, and whether it could be used as an additional electrophysiological quantification of ganglion cell damage.

Methods The PERG, recorded from 30 eyes of 30 patients with different types of clinically and electrophysiologically confirmed optic neuropathies (inclusion criteria: pale optic disc, thinning of retinal ganglion cell layer (RNFL), abnormal PERG N95 wave and abnormal VEP) were retrospectively analyzed and compared to 30 healthy eyes of 30 control subjects. PERG traces were exported and the signal between the P50 and N95 
waves (from 50 to $80 \mathrm{~ms}$ after the stimulus onset) was described by a linear fit ' $y=a x+b$ ', in which the parameter ' $a$ ' indicated the steepness of the slope. Results were compared between both groups with two sample t-test, functional findings were correlated with morphological parameters by Pearson correlation, and sensitivity and specificity were evaluated with receiver-operating characteristic curve (ROC).

Results The patients with optic neuropathy exhibited a significant reduction of the N95 amplitude $(4.3 \pm 1.0$ versus $8.3 \pm 1.1 \mu \mathrm{V}$ in healthy subjects, $p<0.001)$ and reduction of the N95/P50 ratio $(1.0 \pm 0.2$ vs. $1.5 \pm 0.2, p<0.001)$, while P50 amplitude was not affected $(4.5 \pm 1.1$ vs. $5.5 \pm 0.7 \mu \mathrm{V}$, $p=0.68)$. The slope between the P50 and N95 waves was significantly less steep in eyes with optic neuropathies $(-0.089 \pm 0.029$ vs. $-0.220 \pm 0.041, p<0.001)$. When correlating functional and morphological findings, the steepness of the slope showed a significant correlation with temporal RNFL ( $\mathrm{R}=-0.75, p<0.001)$, which was similar as for the N95 amplitude $(\mathrm{R}=0.78, p<0.001)$ and the $\mathrm{N} 95 / \mathrm{P} 50$ ratio $(\mathrm{R}=0.75, p<0.001)$. Based on ROC analysis, temporal RNFL and the slope between the P50 and N95 waves appeared to be the most sensitive and specific parameters for detecting ganglion cell dysfunction, with the largest area under the curve (AUC = 1.0), while AUC of the N95 amplitude and N95/P50 ratio were 0.99 and 0.98 , respectively.

Conclusion The results of this study show that the slope between the P50 and N95 waves is considerably flatter in cases of ganglion cells dysfunction. The steepness of the slope could thus become a useful biomarker of ganglion cell disease and an additional mode of evaluation of the PERG abnormality.

Conflict of interest: None.

Funding: None.

Award applications: None.

Corresponding author email: sustar.majchi@gmail.com

O5.6 Enhancing risk assessment in patients with diabetic retinopathy by combining measures of retinal function and structure

Davis, $Q^{1}$, Chiang, $\mathbf{B}^{2,3}$, Maa, $\mathrm{A}^{3,4}$, Brigell, $\mathrm{M}^{5}$

${ }^{1}$ LKC Technologies, Inc; Gaithersburg, Maryland, USA; ${ }^{2}$ Ophthalmology, Stanford University, Palo Alto, California, USA; ${ }^{3}$ Ophthalmology, Emory University School of Medicine, Atlanta, Georgia, USA; ${ }^{4}$ Regional Telehealth Services, VISN 7, Decatur, Georgia, USA; ${ }^{5}$ Aerpio Pharmaceuticals, Cincinnati, Ohio, USA

Purpose To determine whether or not combining measures of retinal structure and function could predict the need for intervention for diabetic retinopathy (DR) over a 3-year period better than either modality alone.

Methods Subjects were 279 patients with diabetes who participated in an earlier cross-sectional study (NCT01950663 ClinicalTrials.gov); they were selectively recruited to sample a full range of DR severity. Patients were excluded if they were previously treated for macular edema or proliferative DR or if they had other retinopathies. Medical records were reviewed for ocular interventions including vitrectomy, intravitreal injection, pan-retinal photocoagulation, and focal laser. Need for ocular intervention over a 3-year period was analyzed using Kaplan-Meier analyses and Cox proportional hazards. Baseline functional data was obtained in the cross-sectional study with a handheld electroretinogram device, and standard 7-field color fundus photographs were obtained to determine the Early Treatment Diabetic Retinopathy Study (ETDRS) DR severity and macula edema status. Two definitions of structural positive findings were used. First, if patients had ETDRS-DR severity $\geq$ level 53 (ETDRS$\mathrm{DR}+$ ) and second, if patients were ETDRS-DR + or if they had clinically significant macular edema (VTDR + ). A positive function finding corresponded to a RETeval DR score $>23.5$ (RETeval + ).

Results Patients had a $7 \%, 11 \%$, and $17 \%$ probability of needing an ocular intervention in the subsequent 1,2 , and 3 years, respectively. Relative risks (RR) between patients with positive and negative test results for having an ocular intervention were as follows: RETeval + , RR: $11 \times$; VTDR +, RR: $4.2 \times$; ETDRS-DR +, RR: $3.5 \times$. In VTDR + patients there was a $30 \%, 59 \%$, and $83 \%$ probability of an intervention in the subsequent 1, 2, and 3 years, respectively, if function was above criterion (RETeval + ), whereas RETeval- reduced the risk to $3 \%, 3 \%$, and $38 \%$, respectively ( $p=0.001, p<0.001, p=0.009$ ).

Conclusion Prediction of subsequent intervention was best when combining structural and functional information. Combining structural and functional results can improve the management of patients with diabetic eye disease

Conflict of interest: QD is an employee of LKC Technologies, manufacturer of the RETeval device used in this study.

Funding: None.

Award applications: Marmor Award.

Corresponding author email: qdavis@1kc.com

\section{Poster Session 1: Pediatric Electrophysiology}

\section{P1.1 Always keep an open mind - and apply electrophysiology early - a case report}

Palmowski-Wolfe, A, Françoise, $R$

University Hospital Basel, University Eye Hospital, Basel, Switzerland

Purpose To underline the importance of early electrophysiological examination in clinical practice.

Methods Retrospective case report

Results A 16 month old boy with the of diagnosis spasmus was referred for ophthalmologic evaluation. Parents reported episodes of head nodding and nystagmus 20 times per day starting at age 9 months. At 6 months, light sensitivity was noted. An MRI had shown no lesion of the optic path but had shown a small cystic lesion of the pituitary gland compatible with a Rathke Cyst. Examination under anesthesia had shown a healthy aspect of the anterior and posterior eye. Intraocular pressure was normal (OD: $16 \mathrm{mmHg}$; OS: $14 \mathrm{mmHg}$ ). Skiascopy revealed a hyperopia of OD: $+5.50 /-2.50 / 20^{\circ}$; OS: $+5.50 /-2.00 / 7^{\circ}$ ) and glasses were given. Over time head nodding decreased and the intermittent nystagmus (high frequency, small amplitude, mostly pendular but with jerk 
and rotatory components) lessened. Head nodding was no longer seen after the age of 2 years. While the parents and the orthoptist noted increased light sensitivity, and a better orientation in the dark, the ophthalmologist's examinations with a dilated pupil could always be done without signs of light sensitivity. At 33 months, visual acuity with LH Symbols showed a reliable acuity of $0.08 \mathrm{OD}=\mathrm{OS}$. An OCT showed a central retina that seemed well preserved. ERG with skin electrodes was performed which showed no recordable cone or rod activity. Genetic testing revealed an achromatopsia (homozygous variant in CNGB3-gene).

Conclusion 1. Listen to your orthoptist and the parents and also notice when they mention complaints (here: without dilated pupils). 2. Patients can have multiple pathologies. Apply ERG early to catch retinopathies early. In this patient, no treatment options were missed, but when genetic treatment is available, results may be better in early treatment. For achromatopsia, clinical phase 3 treatment trials are running.

Conflict of interest: None.

Funding: None.

Award applications: None.

Corresponding author email: Anja.Palmowski-Wolfe@ usb.ch

\section{P1.2 Hypotrichosis with retinal dystrophy: Electroretinography findings}

Taylan Sekeroglu, $\mathbf{H}^{1}$, Sekeroglu, Mehmet $\mathbf{A}^{2}$

${ }^{1}$ Ophthalmology, Hacettepe University, Ankara, Turkey; ${ }^{2}$ Ophthalmology, Ulucanlar Eye Hospital, Ankara, Turkey

Purpose Hypotrichosis with juvenile macular dystrophy (HJMD, OMIM: 601553) has been described as a rare autosomal recessive disease associated with sparse scalp hair and progressive retinal degeneration caused by CHD3 (cadherin 3) gene mutation located on 16q22.1. We report the fullfield electroretinography findings of a child with hypotrichosis and retinal dystrophy.

Methods A 9 year old boy with decreased visual acuity, nyctalopia and hypotrichosis underwent complete ophthalmological examination including fundus photography, optical coherence tomography (OCT) and flash ERG. The patient was referred for genetic evaluation.

Results The best corrected visual acuity was 20/200 in both eyes. Fundus evaluation showed severe atrophy of the central macular region with pigment clumping and retinal atrophy at the posterior pole in particular. Flash ERG revealed severe reduction of light-adapted and dark-adapted amplitude, the latter more prominent, indicating that the disease affected the entire retina. OCT indicated disruption and loss of inner and outer retinal layers with atrophic changes extending along the posterior pole. A homozygous c.830del mutation was demonstrated on $\mathrm{CDH} 3$ gene.

Conclusion HJMD should be kept in mind in children with early onset retinal dystrophy and congenital hypotrichosis. ERG is indispensable for the diagnosis and may reveal generalized retinal dysfunction despite marked macular involvement.

Conflict of interest: None.
Funding: None.

Award applications: None.

Corresponding author email: h_taylan@yahoo.com

\section{P1.3 Dark- and light-adapted full-field ERG implicit times are shorter in children than in adults}

\author{
Albuhayzah, H, Leat, SJ, McCulloch, D \\ School of Optometry and Vision Science, University of \\ Waterloo, Waterloo, Canada
}

Purpose Understanding normal maturation of retinal function and the acquisition of normal age-related data is fundamental to detect, monitor, and understand pathologic processes that affect the paediatric retina. In the present study, we report darkadapted (DA) and light-adapted (LA) ERGs to full-field white flash stimuli including the ISCEV Standard stimuli in children and compared these to adult ERGs.

Methods Thirty-two participants of European descent with normal ocular and general health were recruited to this crosssectional study. The sample included 12 children between 7 and 11 years, 10 older children and adolescents between 12 and 15 years and 10 adults between 20 and 33 years. Full-field ERGs were recorded simultaneously in each eye from thread electrodes (DTL ${ }^{\circledR}$ fiber) using the Espion E3 system with fully dilated pupils $(0.5 \%$ tropicamide). Stimuli were ISCEV Standard dark-adapted ERGs (DA 0.1, DA 3, DA 10) as well as a LA ERG series with flash strengths of 0.3, 1.0, 3.0, 10 and $24 \mathrm{~cd} \mathrm{~s} / \mathrm{m}^{2}$ (which includes the Standard LA 3.0). We measured a- and b-wave amplitudes and implicit times and used the average of the right and left eye values to compare age groups

Results Spearman's rank correlation was used to determine the relationship between age and ERG implicit time. Both DA and LA a-wave implicit times were significantly positively correlated with age for all stimuli; i.e., implicit times were shorter for children compared to adults. Rank correlations of a-wave with age were $0.588(p=<0.001)$ for the DA 3.0 ERG and $0.707(p<0.001)$ for the DA 10 ERG. Similarly, for LA a-wave ERGs positive rank correlations with age were found for all stimuli (range of $\mathrm{R}=0.352$ to $0.556, p<0.05$ ). B-wave implicit times for both DA and LA ERGs did not correlate with age except for the DA strong and weak flashes $(\mathrm{R}=0.493$, $p=0.04$ and $0.630, p<0.001$, respectively) and LA 1.00 flash ( $\mathrm{R}=0.394, p<0.026$ ). In contrast, ERG DA and LA amplitudes for both a- and b-waves did not significantly differ between the child and adult age groups across all stimuli $(p>0.05$, mixed ANOVAs for DA and LA [age group $\times$ stimulus]).

Conclusion During infancy, ERGs are characterized by long implicit times that undergo a systematic decrease in the first 3 to 5 years of life. For participants aged 7 to approximately 15 years, DA and LA ERG implicit times increase with age and approach adult values. ERG amplitudes are not associated with age, which is in agreement with previous findings that suggested a- and b-wave amplitudes mature sufficiently to be adult-like between 3 and 5 years of age.

Conflict of interest: None.

Funding: Saudi Arabian Cultural Bureau in Canda. 
Award applications: Eberhard Dodt Memorial Award, Marmor Award.

Corresponding author email: daphne.mcculloch@ uwaterloo.ca

\section{Poster Session 2: Optimizing Disease Treatment and Monitoring with the ERG}

\section{P2.1 Electrophysiological monitoring of macular function during the treatment with intravitreal methotrexate for an eye with intraocular lymphoma}

Matsushima, T, Yoshikawa, Y, Shimura, A, Yajima, A, Ojima, Y, Shinoda, K

Ophthalmology, Saitama Medical University, Saitama, Japan

Purpose To describe the recovery of macular function in a case of primary intraocular lymphoma (PIOL) treated with intravitreal injection of methotrexate (ivMTX).

Methods This is a case report describing a patient with intraocular lymphoma treated with ivMTX. Macular function was evaluated using the mfERG recorded using skin electrodes.

Results A 62-year-old man developed color vision deficiency and complained of decreased visual acuity (VA) in his right eye, although VA was 20/20. Fundus examination showed vitreous opacity and multiple subretinal yellowish lesions. Optical coherence tomography (OCT) revealed subretinal and intraretinal infiltrations. The vitreous opacity and infiltrations gradually increased and VA decreased to 20/70. The mfERG recorded using skin electrodes showed severe attenuation of the response density of the central area compared with that of the fellow eye. Then, pars plana vitrectomy, phacoemulsification, and lens implantation were performed for removal of the opacity and for biopsy of the vitreous. The ratio of interleukin10 to 6 was 76.0 and vitreous cytology showed class IIIb lymphoma. There was no systemic malignant lesion and we diagnosed PIOL. Then, ivMTX $(400 \mu \mathrm{g} / 0.1 \mathrm{ml})$ was started. One month after the first ivMTX treatment, intraretinal infiltration disappeared and the mfERG showed recovery of the response density of the central area. Two months later, VA recovered to 20/17 and mfERG showed maintenance of macular function.

Conclusion The mfERG recorded using skin electrodes was useful for monitoring macular function with repeated examinations and showed recovery of macular function in a case of PIOL treated with ivMTX.

Conflict of interest: None.

Funding: None.

Award applications: Eberhard Dodt Memorial Award, Marmor Award.

Corresponding author email: takashi030734@yahoo.co.jp
P2.2 Electroretinographic monitoring of macular function during internal limiting membrane peeling

Gaku, $\mathbf{T}^{\mathbf{1}}$, Shinoda, $\mathbf{K}^{\mathbf{1 , 2}}$, Yagura, $\mathbf{K}^{\mathbf{1}}$, Kawashima, $\mathbf{M}^{\mathbf{1}}$, Nakamura, $\mathrm{N}^{1}$, Matsumoto, $\mathrm{C} \mathrm{S}^{1,3}$, Mizota, $\mathrm{A}^{1}$, Miyake, $\mathbf{Y}^{4}$

${ }^{1}$ Department of Ophthalmology, Teikyo University School of Medicine, Tokyo, Japan; ${ }^{2}$ Department of Ophthalmology, Saitama Medical University Faculty of Medicine, Saitama, Japan; ${ }^{3}$ Matsumoto Eye Clinic, Osaka, Japan; ${ }^{4}$ Department of Ophthalmology, Aichi Medical University School of Medicine, Aichi, Japan

Purpose The purpose of this study was to evaluate the influence of Brilliant Blue G (BBG)-assisted internal limiting membrane (ILM) peeling on macular function using the intraoperative focal macular electroretinogram (iFMERG).

Methods Fourteen eyes of 14 patients who underwent 25 gauge vitreous surgery with ILM peeling using fourfold diluted BBG dye were included. Patients consisted of nine males and five females and the mean age was $68.8 \pm 9.14$ years old. Three eyes had macular holes and 11 eyes had epiretinal membranes. The iFMERG was recorded according to the protocol stated previously (Matsumoto et al. PloS One 2015; https://doi.org/10.1371). The recording was performed three times during surgery. The first recording was performed before BBG dye was injected as baseline (Phase 1). The second recording was performed just after the BBG was washed out (Phase 2). The third recording was performed after ILM peeling (Phase 3). iFMERG was recorded twice at each phase. The implicit time and the amplitude were measured for a-wave, b-wave, photopic negative response (PhNR), and d-wave. Paired t-test was used to compare phase 2 and phase 3 with phase 1 as baseline and the significance level was set at $p<0.05$.

Results The mean implicit time of the a-wave was $24.9 \pm 3.50,25.5 \pm 3.96,24.0 \pm 3.46 \mathrm{~ms}$ at Phases 1, 2, and 3 , respectively. That of the b-wave was $45.2 \pm 3.71$, $44.7 \pm 4.61,44.4 \pm 4.37 \mathrm{~ms}$ at each Phase, respectively. That of the PhNR was $67.6 \pm 6.48,67.7 \pm 5.87,66.0 \pm 5.13 \mathrm{~ms}$ at each Phase, respectively. That of d-wave was $128.0 \pm 4.24$, $127.4 \pm 2.99,127.3 \pm 5.58 \mathrm{~ms}$ at each Phase, respectively. No significant difference was observed between Phases 1 and 2 , and between Phases 1 and 3. The mean amplitude of the a-wave was $2.12 \pm 1.28,1.86 \pm 0.95,2.03 \pm 1.33 \mu \mathrm{V}$ at each Phase, respectively. That of $b$-wave was $5.33 \pm 3.05$, $4.97 \pm 2.41,5.21 \pm 3.16 \mathrm{uV}$ at each Phase, respectively. That of PhNR was $1.89 \pm 1.18,1.60 \pm 0.88,1.67 \pm 1.03 \mu \mathrm{V}$ at each Phase, respectively. That of d-wave was $1.94 \pm 0.96$, $1.70 \pm 0.84,1.88 \pm 1.02 \mu \mathrm{V}$ at each Phase, respectively. No significant difference was observed between Phases 1 and 2 and between Phases 1 and 3 .

Conclusion The results showed that the BBG dye as well as the ILM peeling procedure were safe with regard to retinal function. iFMERG is useful for evaluating the influence of each surgical procedure on macular function, proving its safety.

Conflict of interest: None.

Funding: None.

Award applications: Marmor Award. 
Corresponding author email: jakkaruniomakase22@ yahoo.co.jp

P2.3 A 3-year longitudinal study of electroretinogram and optical coherence tomography findings in patients with multiple sclerosis

Hanson, James V. M. ${ }^{1}$, Nh, Mei-Yee ${ }^{2}$, HaywardKönnecke, Helen K. ${ }^{3}$, Schippling, Sven ${ }^{4}$, Reeve, Kelly A. ${ }^{2}$, Gerth-Kahlert, Christina ${ }^{1}$

${ }^{1}$ Ophthalmology, University Hospital Zurich and University of Zurich, Zurich, Switzerland; ${ }^{2}$ Epidemiology, Biostatistics and Prevention Institute, Department of Biostatistics, University of Zurich, Zurich, Switzerland; ${ }^{3}$ Neuroimmunology and Multiple Sclerosis Research, Department of Neurology, University Hospital Zurich and University of Zurich, Zurich, Switzerland; ${ }^{4}$ Neuroscience Center Zurich, University of Zurich and Swiss Federal Institute of Technology Zurich, Zurich, Switzerland

Purpose Researchers have in recent years begun to investigate ocular effects of multiple sclerosis (MS) other than optic neuritis $(\mathrm{ON})$, and it is now clear that changes to both retinal function (measured using the ERG) and structure (measured using optical coherence tomography, OCT) are found in patients with MS irrespective of a history of ON. The majority of studies to date have presented only cross-sectional data regarding ERG and OCT. We therefore studied the longitudinal course of ERG and OCT in patients with MS, as well as the effect of recent clinical relapses on these functional and structural measures.

Methods Participants in an ongoing longitudinal study of MS were invited to take part in a 3-year ophthalmological substudy. Exclusion criteria were ophthalmological or neurological disease other than MS, diabetes mellitus, and hyperopia or myopia $>6$ diopters. Baseline examinations consisted of subjective refraction, high- and low-contrast visual acuity, ERG, and OCT. Measures of MS status such as Expanded Disability Status Scale (EDSS) score and relapse history were obtained by neurological examination. These examinations were repeated annually for 3 years. Examinations took place between June 2014 and October 2019. ERG peak times, rod ERG b-wave amplitude, mixed rod/cone and cone b/a wave amplitude ratios, thickness of the peripapillary retinal nerve fibre layer, and volumes of the segmented retinal layers/complexes were included in the analyses. Using generalized estimating equation models adjusted for age, ON, and MS treatment status, we assessed changes to ERG and OCT parameters over the study duration, the effect of MS relapses in the preceding year on ERG and OCT parameters, and the effect of selected OCT parameters (inner nuclear layer, INL; outer nuclear layer, ONL; outer retinal layers, ORL) on corresponding ERG parameters.

Results After dropouts, 23 patients (45 eyes, as one eye of one patient was excluded due to exotropia) were included in the longitudinal analyses. OCT or ERG information was missing for two, seven, ten, and one eyes at baseline and at 1,2, and 3 years, respectively. Significant changes to several ERG peak times over the study period were recorded, however the effect sizes were very small (typically less than the $0.5 \mathrm{~ms}$ temporal resolution of the ERG device). OCT values remained relatively stable over the course of the study. No evidence of an effect of MS relapses in the previous year on ERG or OCT parameters was observed. Little to no evidence of associations between INL, ONL, or ORL and ERG parameters was observed.

Conclusion MS relapses within the previous year appear not to affect ERG and OCT outcome measures. As ERG changes in MS patients over 3 years are likely to be small and of questionable clinical relevance, longitudinal studies of retinal function in MS should be planned over an extended period.

Conflict of interest: None.

Funding: Velux Stiftung; Bruppacher Stiftung.

Award applications: None.

Corresponding author email: james.hanson@usz.ch

\section{Poster Session 3: Assessing Retinal Function in Acquired Retinopathies}

P3.1 Correlations between macular microvascular structure and macular function evaluated by optical coherence tomography angiography and multifocal electroretinogram in high myopia patients

Kim, Hoon Dong', Woo, Sang Earn², Han, Jung Woo², Ohn, Young-Hoon ${ }^{2}$

${ }^{1}$ Department of Ophthalmology, Soonchunhyang University College of Medicine, Cheonan, Korea; ${ }^{2}$ Department of Ophthalmology, Soonchunhyang University College of Medicine, Bucheon, Korea

Purpose To investigate the correlations between macular function recorded with mfERG and macular microvascular structure evaluated with optical coherence tomography angiography (OCT-A) in patients with high myopia.

Methods Twenty eyes in 12 patients with high myopia without pathologic change and 30 eyes of 15 healthy subjects were enrolled in this study, and all subjects underwent OCT-A scanning and mfERG recording. In addition, ocular characteristics including best-corrected visual acuity (BCVA), refractive error and axial length were also reviewed. The OCT-A images were obtained with the Cirrus ${ }^{\circledR}$ HD-OCT 5000 with AngioPlex OCT-A (Carl Zeiss Meditec, Inc; Dublin, CA), and an installed automated program was used to analyse the microvascular patterns at each layer of the macula. The area and circularity of foveal avascular zone (FAZ) were measured, and vessel density (VD) and perfusion density (PD) of superficial and deep capillary plexus (SPC, DPC) were also estimated. Moreover, the mean retinal thickness, central subfield thickness, and retinal nerve fiber layer (RNFL) thickness were also measured from OCT-A. The averaged amplitudes and implicit times of N1, P1 and N2 in Ring 1 and 2 were obtained from mfERG parameters. mfERG recordings were carried out in accordance with the ISCEV Standard protocol for mfERG. The correlations between OCT-A results and mfERG parameters were analysed in high myopia patients and healthy subjects.

Results The mean FAZ areas in high myopia patients were increased significantly compared with healthy subjects $(p=0.038)$. Both PD and VD in the SPC and DPC were 
significantly reduced in high myopia patients compared to healthy subjects $(p<0.001, p<0.001$, respectively). The averaged amplitudes of N1, P1, and N2 from Ring 1 and Ring 2 were reduced significantly in high myopia patients compared with healthy subjects. Both PD and VD of the superficial plexuses were also significantly correlated with $\mathrm{N} 1, \mathrm{P} 1$, and N2 amplitudes of mfERG.

Conclusion The averaged N1, P1, and N2 amplitudes of mfERG were significantly correlated with PD and VD of SPC and DPC in high myopia patients. All amplitudes of mfERG may be more correlated with OCT-A findings compared with implicit time. This suggests that OCT-A with mfERG can be a useful modality to evaluate both macular microvascular structure and macular function in high myopia patients.

Conflict of interest: None.

Funding: Velux Stiftung; Bruppacher Stiftung.

Award applications: None.

Corresponding author email: james.hanson@usz.ch

P3.2 Comparison of macular function before and after micropulse subthreshold coagulation for diabetic macular edema using skin electrode multifocal electroretinogram

Takano, $\mathbf{S}^{\mathbf{1}}$, Ozaki, $\mathbf{K}^{\mathbf{1}}$, Yoshikawa, $\mathbf{Y}^{\mathbf{1}}$, Kumagai, $\mathbf{T}^{\mathbf{1}}$, Ishii, $\mathbf{H}^{1}$, Sasaki, $\mathbf{T}^{\mathbf{1}}$, Igawa, $\mathbf{Y}^{\mathbf{1}}$, Kanno, $\mathbf{J}^{\mathbf{1}}$, Ibuki, $\mathbf{H}^{1}$, Shimura, $A^{1}$, Nagashima, $T^{2}$, Shoji, $T^{1}$, Shinoda, $K^{1}$

${ }^{1}$ Ophthalmology, Saitama Medical University, Moroyama, Japan; ${ }^{2}$ Ophthalmology, Kamishirane Hospital, Yokohama, Japan

Purpose To investigate the changes in macular response density before and after subthreshold micropulse coagulation (SDM) for diabetic macular edema (DME) using mfERG.

Methods Patients with DME who underwent SDM at Saitama Medical University Hospital between August 2019 and June 2020 were retrospectively reviewed. The mfERG was recorded using skin electrodes before and one month after the SDM. The amplitudes of $\mathrm{N} 1$ and $\mathrm{P} 1$ and latencies in the response from the central area with a diameter of $3 \mathrm{~mm}$ were analyzed. To compare the value of response density before and after SDM, Wilcoxon signed rank test was conducted.

Results Nine subjects were analyzed in this study. Median age (quartile) was $72(65.5,75)$. Subjects were nine eyes of seven cases (three males, four females), age $70.7 \pm 8.4$ years [mean \pm standard deviation]). The amplitude before SDM was P1 $5.93(4.46,7.83) \mathrm{nV} /$ degree $^{2}$ (median [interquatile range]); $\mathrm{N} 1-2.3(-3.74,0.07)$, and after SDM was P1 7.05 $(4.56,9.44)$; N1 $-3.43(-5.08,-1.54)$, which was not a significant change. The latency before SDM was P1 32.71 $(30.73,38.54) \mathrm{msec}$; N1 $17.29(13.86,19.32)$, and after SDM was P1 29.9 (27.19, 41.67); N1 15.73 (11.36, 17.71), which was significantly improved in N1 after SDM ( $p=0.0156)$.

Conclusion mfERG using skin electrode was useful for evaluating macular function before and after treatment. Our study suggested that SDM improved outer retinal function in patients with DME.

Conflict of interest: None.

Funding: None.
Award applications: Eberhard Dodt Memorial Award. Corresponding author email: smumshun10@gmail.com

\section{P3.3 Symmetry of first order kernel mfERG response in patients screened for HCQ maculopathy}

Tzekov, R, Kiri, G, Bhaleeya, S

Department of Ophthalmology, University of South Florida, Tampa, FL, USA

Purpose Evaluating ring ratios in mfERG recording is now a common practice used in the process of screening patients for development of maculopathy associated with prolonged use of hydoxycholoroquine (HCQ). Typically, the N1P1 amplitude is used to estimate the ring ratio amplitude. The purpose of this work was to evaluate to what extent N1P1 is correlated with P1N2 amplitude and how this affects the calculation of ring ratios, specifically $\mathrm{R} 1 / \mathrm{R} 2$ ratio.

Methods This was a retrospective study of mfERG records of patients screened for HCQ maculopathy during the period January 2015 to December 2019 at the USF Eye Institute in Tampa, FL. Only internal referrals and the records of patients with more than one mfERG recording (less than 2 years after first recording) were evaluated. Recordings were made binocularly using DTL electrodes on an Espion mfERG system (Diagnosys LLC, Lowell, MA) using a standard 61-hexagon, $60^{\circ}$ stimulus, 14 bit m-sequence, bandpass $10-100 \mathrm{~Hz}$, no spatial averaging and moderate smoothing (level 3). NIP1 amplitude (referred as P1) and P1N2 amplitude (referred as N2) were evaluated for both ring 1 and ring 2 .

Results The records of 10 patients, all females, age $56.3 \pm 11.3$ years at time of first mfERG testing, were available for analysis. The average time between first and second testing was $0.8 \pm 0.3$ yrs. The amplitudes of P1 and N2 were highly correlated (Pearson $\mathrm{R}=0.65-0.97, p<0.05$ ) for both rings, eyes and visits. That was reflected also in similar decrease in amplitude from visit 1 to visit 2 , range $-4.6 \%$ to $-36 \%$, but no difference between P1 and N2 $(p>0.05$, paired t-test) for both rings and eyes. That similarity was further reflected in similarity in $\mathrm{R} 1 / \mathrm{R} 2$ ratio with no difference between the ratios calculated on the basis of P1 vs. N2 amplitudes $(p>0.05)$.

Conclusion The ring ratio based on $\mathrm{N} 2$ amplitude may be a useful addition to the traditionally used ratios based on P1 amplitude. Further evaluation of additional ring ratios and a large sample size would provide additional insight about the dynamics of mfERG changes in HCQ maculopathy screening. Conflict of interest: None.

Funding: None.

Award applications: None.

Corresponding author email: rtzekov@usf.edu

P3.4 Who is receiving mfERG testing for anti-malarial retinopathy? A survey of a referral practice

Wright, Thomas ${ }^{1,2}$, Christakis, PG $^{2,3}$

${ }^{1}$ Kensington Vision and Research Center, Toronto, Canada;

${ }^{2}$ Dept. Ophthalmology and Vision Sciences, University of 
Toronto, Toronto, Canada; ${ }^{3}$ Toronto Western Hospital, Toronto, Canada

Purpose To better understand which patients are being referred for mfERG in the context of screening for anti-malarial retinopathy. The mfERG is a useful test for identifying retinal toxicity caused by hydroxychloroquine/chloroquine (HCQ/CQ) therapy. Access to mfERG testing is often limited and the sensitivity and specificity for detection of early retinopathy is unclear. Prevalence estimates for retinopathy, when based on mfERG testing, are often higher than for other testing modalities. This may represent a selection bias, in that the population referred for mfERG screening does not represent the wider population taking anti-malarial therapies.

Methods All physicians and healthcare professionals referring patients taking $\mathrm{HCQ} / \mathrm{CQ}$ for mfERG testing at the Kensington Vision and Research Centre were asked to complete an anonymous online survey. The survey assessed their approach to retinopathy screening for patients at baseline, between 1 and 5 years and after more than 5 years of therapy. The types of screening tests used in regular practice as well as the risk factors prompting referrals for mfERG screening were assessed. Questions were presented using five level Likert scales (Always, Often, Sometimes, Rarely, Never) or 3 level (Increase, Same, Decrease) Likert scales.

Results 34 healthcare professionals were asked to complete the survey, and complete responses were received from $67 \%$ of those surveyed (23/34). All respondents started screening patients before therapy had continued for 5 years. At baseline, $17 \%(4 / 23)$ of respondents requested mfERG testing "always" or "often". After 5 years of therapy, 56\% (13/23) of respondents requested mfERG testing "always" or "often". At baseline, $65 \%$ of respondents "never" requested mfERG screening for patients without risk factors for toxicity although this decreased to $9 \%$ for patients with abnormal results on other screening tests and $17 \%$ for patients with other risk factors. Differentiating age-related macular degeneration from early retinopathy and unreliable $\mathrm{HVF}$ were listed as the two most common reasons for requesting mfERG testing. Difficulty accessing mfERG testing was also listed as a factor limiting referrals.

Conclusion mfERG testing for patients taking HCQ/CQ therapy is not routinely requested at baseline. Patients are more likely to be referred for testing if the physician already suspects retinal pathology due to abnormal results on other screening tests or due to the presence of other risk factors. Care needs to be taken when considering $\mathrm{HCQ} / \mathrm{CQ}$ prevalence measures based on mfERG testing as the population may not be representative of the general population.

Conflict of interest: None.

Funding: None.

Award applications: None.

Corresponding author email: twright@kensingtonhealth.org
P3.5 Variation analysis of the mfERG may aid early detection of retinopathy induced by anti-malarial therapy

Habib, $\mathbf{F}^{1,2}$, Huang, $\mathbf{H}^{2,3,4,5,6}$, Gupta, $\mathrm{A}^{2}$, Wright, $\mathrm{T}^{1,7}$

${ }^{1}$ Kensington Vision and Research Centre, Toronto, Canada; ${ }^{2}$ Dept. Computer Science, University of Toronto, Toronto, Canada; ${ }^{3}$ Dept. of Math, York University, Toronto, Canada; ${ }^{4}$ Fields CQAM Health Analytics and Multidisciplinary Modeling Lab, Toronto, Canada; ${ }^{5}$ Advanced Institute of Natural Science, Beijing Normal University at Zhuhai, China; ${ }^{6} \mathrm{BNU}-\mathrm{HKBH}$ United International College, Zhuhai, China; ${ }^{7}$ Dept. Ophthalmology and Vision Sciences, University of Toronto, Toronto, Canada

Purpose The use of anti-malarial drugs, hydroxychloroquine (HCQ) and chloroquine (CQ), for treatment of systemic autoimmune disease is widespread. Studies have shown the development of retinal toxicity is a significant risk particularly with long duration therapy and high cumulative dosage. If not detected at an early stage, retinal toxicity can become irreversible and progressive, eventually affecting the patient's sight. The development of sensitive quantitative screening tests is essential to maximize the safety of $\mathrm{HCQ} / \mathrm{CQ}$ retinopathy. The multifocal electroretinogram (mfERG) is an objective test of retinal function sensitive to early changes caused by early HCQ/CQ toxicity. Ring ratio analysis is a commonly applied quantitative analysis that compares response amplitudes at different eccentricities from the fovea. This analysis is most sensitive when toxicity presents as preserved foveal function with a para-central ring of function loss, a Bulls-eye pattern. This study will examine other quantitative measures based on the mfERG to determine their ability to detect early HCQ/CQ retinopathy.

Methods All patients taking HCQ or CQ who were referred to the visual electrophysiology service at Kensington Vision and Research Centre, Toronto, Canada (KVRC) for mfERG testing between August 2017 and December 2019 were identified. mfERG testing was performed using the Espion system (Diagnosys LLC; MA, USA) with a 61 hexagon protocol according to ISCEV Standards. Custom software was developed to enable direct access to mfERG trace data stored in the recording system proprietary database. Ring ratio analysis was applied to identify subjects demonstrating signs of retinal toxicity. mfERG variability was measured by subtracting an eccentricity dependent average waveform "ring average" from individual responses within a ring. Residuals are summed and the result compared with ring ratio measures. Correlation between the two measures was assessed both visually using Receiver Operator Characteristic (ROC) curves and using an analysis of variance with a "within subject" (eye) and "within test" (eccentricity) measure.

Results mfERG tests were extracted from 722 patients. Patient sex was unequally distributed $(87.5 \%$ female), Median patient age was 57 years [male 65 (range 31 to 86) and female 56 years (range 19 to 93 ) female]. $51 \%$ of eyes were identified as showing toxicity with ring ratio analysis. mfERG variability was significantly higher $(p \ll 0.01)$ in subjects demonstrating toxicity with the ring ratio analysis compared to subjects without toxicity $(\mathrm{AUC}=0.60)$. 
Conclusion Variance analysis of the mfERG is sensitive to changes caused by HCQ/CQ therapy. Further study is required to determine if this approach can be developed into a sensitive, quantitative screening test.

Conflict of interest: None.

Funding: None.

Award applications: None.

Corresponding author email: twright@kensingtonhealth.org

\section{P3.6 Characterisation of retinal dysfunction associated with quinine toxicity in 20 patients}

Georgiou, $A^{1}$, Neveu, $M^{1,2}$, Calcagni, $A^{1,2}$, Robson, $A^{1,2}$

${ }^{1}$ Electrophysiology Department, Moorfields Eye Hospital, London, UK; ${ }^{2}$ Institute of Ophthalmology, University College London, London, UK

Purpose To describe the electrophysiological findings in patients with suspected quinine toxicity.

Methods This is a retrospective review of 23 patients referred to the Moorfields Eye Hospital Department of Electrophysiology with either suspected quinine retinal toxicity or with electrophysiological findings consistent with this diagnosis and/or a positive past medical history of quinine intake. ISCEV Standard pattern (PERG) and full-field electroretinogram (ERG) testing was performed in all patients. Four subjects also underwent electrooculography (EOG). Six patients also had follow-up electrophysiology.

Results Twenty patients had ERG results consistent with quinine toxicity. Eight individuals had visual acuity of $\geq 6 / 9$ bilaterally; 18 had visual acuity $\geq 6 / 18$ in both eyes. The PERG P50 component was undetectable/residual in 15 patients and subnormal in the remaining five. The DA 0.01 ERG was subnormal in 18 cases. All patients showed normal DA 10 a-wave amplitude; the b:a ratio was subnormal in 19 cases (mean 0.75 ; range 0.5 to 1.5 ), with an electronegative waveform in 18 of these. The LA $30 \mathrm{~Hz}$ ERG was always subnormal and was delayed in 19 cases. The LA 3.0 ERG was abnormal in all cases, with reduced b:a ratio in 19 of 20 patients. The on-off ERG b:a ratio was reduced in 17 of the 19 cases where this was performed; most patients showed additional off response abnormality, with a positive plateau following the d-wave peak. All patients who underwent additional EOG testing had a light peak to dark trough ratio that was more severely reduced than would be expected from the ERG findings alone. Three of the six patients who had follow-up visits showed deterioration of retinal function over time, including DA 10 ERG b-wave reduction in the one case with a previously normal b:a ratio.

Conclusion Quinine toxicity is associated with generalised rod and cone system dysfunction at a level that is post-phototransduction. On-off ERGs show an electronegative on-response and frequently a distinctive off-response abnormality, indicative of ON- and OFF-bipolar pathway involvement, respectively. There is PERG evidence of macular dysfunction in all cases, including those with preserved visual acuity, and EOG evidence of additional RPE dysfunction in some cases. The findings highlight the importance of electrophysiology in the diagnosis and monitoring of quinine toxicity.
Conflict of interest: None.

Funding: None.

Award applications: None.

Corresponding author email: anne.georgiou1@nhs.net

P3.7 Differences between rheumatoid arthritis and systemic lupus erythematosus patients on long-term Plaquenil therapy

\section{Xu, M, MacDonald, I}

Ophthalmology \& Visual Sciences, University of Alberta, Edmonton, Canada

Purpose To define the characteristics of patients resilient to high cumulative doses of Plaquenil.

Methods From patients referred to a single-centre referral practice and screened for Plaquenil retinopathy, those who received cumulative doses of more than $2 \mathrm{~kg}$ were identified prospectively over a 16-month period (December 1, 2018 to April 1, 2020). All patients underwent multifocal electroretinography (mfERG), fundus photography, fundus autofluorescence and spectral-domain optical coherence tomographic (OCT) imaging as part of their visit. Data collected were: clinical diagnosis, age, sex, daily dose, cumulative Plaquenil dose, duration of use, co-morbid maculopathy, and tamoxifen use. Patients with co-morbid maculopathy were excluded from the analysis.

Results During the study period, 17 patients with rheumatoid arthritis (RA) and 18 with systemic lupus erythematosus (SLE) received a total dose of more than $2 \mathrm{~kg}$ of Plaquenil and had no evidence of Plaquenil retinopathy as measured by fundus photography, autofluorescence imaging, OCT imaging or mfERG when compared with normative data. A comparison between these two groups of resilient subjects (RA vs SLE) revealed that they were similar in terms of age (62 vs 59 years, $p=0.391$ ), sex ( $82 \%$ vs $100 \%$ were females, $p=0.062$ ), daily dose $(5.02$ vs $4.72 \mathrm{mg} / \mathrm{kg}$ real body weight, $p=0.567)$, cumulative dose (2.57 vs $2.64 \mathrm{~kg}, p=0.663)$, and duration of use (18.3 vs 20.5 years, $p=0.197)$. None of them were on tamoxifen. Also, there was no significant difference in terms of mfERG amplitudes: ring 1 (16.8 vs $17.2 \mathrm{nV}, p=0.864)$, ring 2 (10.2 vs $10.5 \mathrm{nV}, p=0.843$ ), ring 3 (6.6 vs $6.7 \mathrm{nV}, p=0.895$ ), and ring ratio (1/3) (2.6 vs 2.6, $p=0.769)$. However, Plaquenil resilient subjects accounted for $14 \%$ of all patients with RA (125) and 35\% with SLE (51) screened during the same period, which were significantly different from each other, $\mathrm{X}^{2}(1$, $\mathrm{N}=176)=10.7, p=0.001$.

Conclusion While this is a small study and there may be bias in the referral of cases for testing, there would appear to be a significant difference between patients as to their relative resilience to Plaquenil toxicity based on their underlying clinical diagnosis.

Conflict of interest: None.

Funding: None.

Award applications: None.

Corresponding author email: macdonal@ualberta.ca 
P3.8 Early detection of neurodegeneration in type 2 diabetic patients without diabetic retinopathy using electroretinogram and spectral-domain optical coherence tomography

\author{
Abdelshafy, Marwa ${ }^{1}$ \\ Department of Ophthalmology, Benha University, Benha, \\ Egypt
}

Purpose To assess early functional and structural changes in the neural retina in type 2 diabetic patients with no clinical signs of diabetic retinopathy (DR).

Methods 30 eyes of 16 diabetic patients without clinical DR, with a mean age of $48.75 \pm 3.09$ years (group 1), 30 eyes of 16 diabetic patients with DR, with a mean age of $51.75 \pm 2.86$ years (group 2) and a control group of 32 eyes of 16 healthy age and sex matched subjects, with a mean age of $49.88 \pm 4.26$ years were included in this study. After full ophthalmologic examination, optical coherence tomography scans, multifocal electroretinogram (mfERG), pattern electroretinogram (PERG), and photopic negative response (phNR) were tested for all participants. Statistical analysis was performed to compare ganglion cell complex (GCC) thicknesses, mfERG, PERG, and phNR values between groups.

Results Significant differences of total GCC thickness were found between group $1 \quad(107.13 \pm 7.04 \mu \mathrm{m})$, group 2 $(97.27 \pm 10.97 \mu \mathrm{m})$ and control group $(113.81 \pm 5.26 \mu \mathrm{m})$ $(p<0.001)$. Significant differences of mfERG foveal P1 wave, PERG N95, and phNR values were found between groups $(p<0.001)$. The inferior quadrant GCC thickness ROC curve had an AUC value $(0.831, p<0.001)$. The sensitivity and specificity for a cut-off point of $100.5 \mu \mathrm{m}$ were $93.3 \%$ and $66.7 \%$, respectively. This was followed by the superior quadrant (GCC thickness AUC value 0.753). The PERG N95 amplitude had the widest AUC (0.931 with $100 \%$ sensitivity, $86.7 \%$ specificity), followed by the phNR amplitude ( 0.90 with $100 \%$ sensitivity, $66.7 \%$ specificity).

Conclusion Preclinical DR presents with neural loss in the macular area evident by the reduction in GCC thickness and impairment of mfERG, PERG, and phNR parameters. Neurodegenerative changes may precede the microvascular damage in these patients. GCC thickness, PERG, and phNR values may prove to be a useful biomarker for early detection of neurodegeneration in diabetic patients and may also serve as an indicator of disease progression.

Conflict of interest: None.

Funding: None.

Award applications: None.

Corresponding author email: drmarwatabl2012@gmail.com

\section{Poster Session 4: Recording the ERG: Technical Issues and Novel Approaches}

\section{P4.1 Probing human cone electroretinogram responses shortly after onset of intense backgrounds}

Rufus-Toyeiang, $\mathbf{R}^{1,2}$, Jiang, $\mathbf{X}^{2,3}$, Margetts, $M^{1}$, Chow, $\mathbf{I}^{3}$, Indusegaran, $\mathbf{M}^{3}$, Webster, $\mathbf{A R}^{2,4}$, Hammond, $\mathbf{C J}^{3}$, Mahroo, $\mathbf{O}^{1,2,3,4}$

${ }^{1}$ Physiology, Development \& Neuroscience, University of Cambridge, Cambridge, UK; ${ }^{2}$ Institute of Ophthalmology, University College London, London, UK; ${ }^{3}$ Section of Ophthalmology, King's College London, St Thomas' Hospital Campus, London, UK; ${ }^{4}$ Moorfields Eye Hospital, London, UK

Purpose Prior electroretinogram (ERG) studies suggest that, at steady state, in intense backgrounds sufficiently strong to bleach $\sim 90 \%$ of the photopigment, cones may preserve up to half the dark-adapted circulating current (the latter measured on a dim rod-saturating background). A postulated mechanism is that the reduced quantal catch means that the background elicits fewer photo-isomerisations and thus less suppression of light-sensitive current. We hypothesised that at early times post background onset, prior to establishment of a steady-state bleach, circulating current would be suppressed below this level.

Methods ERGs were recorded from four healthy adults (after pharmacological mydriasis), in response to strong white xenon flashes (50 photopic $\mathrm{cd} \mathrm{s} / \mathrm{m}^{2}$ ), using conductive fibre electrodes (lower conjunctival fornix). Strong white backgrounds were presented for $2 \mathrm{sed}$; a white xenon flash was delivered $1.5 \mathrm{~s}$ after background onset. A blue rod-saturating background of 30 scotopic $\mathrm{cd} / \mathrm{m}^{2}$ was continually present. The procedure was repeated every $5 \mathrm{~s}$; flash responses were averaged to improve the signal-to-noise ratio. The breaks from background presentation prevented cumulative bleaching. The photopic ERG a-wave represents the cone response but also contains postreceptoral signals. A patient with prior quinine toxicity, in whom post-receptoral neurons had been lost, was recruited to undergo the same experimental protocol.

Results ERG a-waves were progressively smaller in brighter backgrounds, consistent with reduction in available cone circulating current. The form of the relationship resembled that seen in previous direct recordings from macaque cones. The a-wave amplitude at $7 \mathrm{~ms}$ post-flash on the strongest background $\left(2000 \mathrm{~cd} / \mathrm{m}^{2}\right)$ was normalised to the dark-adapted (blue background) value to estimate fractional circulating current. In four healthy subjects, mean (SEM) estimated 
fractional current ranged from $0.03(0.04)$ to $0.18(0.06)$. Recordings from the patient with prior quinine toxicity on a $1500 \mathrm{~cd} / \mathrm{m}^{2}$ background yielded an estimated fractional current of $0.22(0.12)$.

Conclusion We found that in healthy subjects, the photopic ERG a-wave is suppressed to well below $50 \%$ at early times following onset of a background bright enough to yield an estimated $90 \%$ steady state bleach. The findings from the patient with loss of post-receptoral neurons confirm that suppression is at the level of the cone light-sensitive current. These data are consistent with the subsequent reduction in quantal catch at later times permitting recovery of current and thus facilitating vision in the presence of such intense backgrounds.

Additional information

Conflict of interest: None.

Funding: Wellcome Trust.

Award applications: Eberhard Dodt Memorial Award.

Corresponding author email: o.mahroo@ucl.ac.uk

\section{P4.2 Identification of the ERG i-wave: can it be achieved} for all i-wave morphologies?

Ha, $\mathbf{J}^{1,2}$, Sarossy, $M^{1,3,4}$

${ }^{1}$ Centre for Eye Research Australia, East Melbourne, VIC, Australia; ${ }^{2}$ Alfred Health, Melbourne, VIC, Australia; ${ }^{3}$ University of Melbourne, Parkville, VIC, Australia; ${ }^{4}$ Royal Victorian Eye and Ear Hospital, East Melbourne, VIC, Australia

Purpose Extraction of ERG features enables the utilisation of machine learning techniques to enhance the diagnosis and monitoring of patients with retinal diseases. While the a- and b-waves are well known and automated detection is routine, the $\mathrm{i}$-wave is an additional feature that may have future utility. It is defined as the first upward inflection after the b-wave and is thought to indicate a cone-mediated response from the inner retina and retinal ganglion cells. The purpose of this study is to develop an algorithm that can automate identification of the i-wave in electroretinography (ERG) and to facilitate classification of its morphologies.

Methods Testing was conducted using the Espion E3 (Diagnosys, Lowell, MA) with the ColorDome stimulator. 107 eyes of 57 patients (age range 44-92 years, mean 71) with glaucoma (Central 24-2 field testing mean deviation range +2.29 to $-30.62 \mathrm{~dB}$, mean $-4.51 \mathrm{~dB}$ ) were recruited to take part in the study. DTL thread electrodes were applied, and eyes were dilated with tropicamide prior to $5 \mathrm{~min}$ of pre-adaption. Visual stimulation consisted of two steps: $4 \mathrm{~ms}$ light pulses of (1) $1 \mathrm{~cd} \mathrm{~s} / \mathrm{m}^{2}$ red on a $10 \mathrm{~cd} / \mathrm{m}^{2}$ constant homogenous blue background, and (2) $1 \mathrm{~cd} \mathrm{~s} / \mathrm{m}^{2}$ white- $6500 \mathrm{~K}$ on a $10 \mathrm{~cd} / \mathrm{m}^{2}$ white-6500 K background. 100 sweeps were recorded for each step at a $4 \mathrm{k} \mathrm{Hz}$ sample rate, with intersweep delays of $200 \mathrm{~ms}$ and recording epoch from $-20 \mathrm{~ms}$ to $250 \mathrm{~ms}$ relative to ERG stimulus onset. The dataset and the location of the i-wave were validated by two independent clinicians. Poor traces $(n=10)$ manifesting as an undetectable a-wave or b-wave were manually excluded from the analysis. Altogether, 442 separate ERG traces were recorded from participants; the final traces for data analysis were taken as the average trace of all 100 sweeps. An algorithm was developed using $\mathrm{R}$ to identify the i-wave from these averaged traces. In brief, a two-stage technique was used: a local minimisation/maximisation (MinMax) method was used, cascading to a differencing technique in the absence of a concave down turning point. Success was defined as the identification of the i-wave location within $5 \mathrm{~ms}$ on either side of the manually selected true i-wave location.

Results MinMax detected the i-wave in 423 of the 442 averaged traces. In each of these, the i-waves consistently had a 'notched' morphology (a clear concave down turning point after the b-wave). The remaining 19 curves were consistently a 'slurred' morphology (a descending non-stationary point of inflection), and all were detected by the differencing technique within the algorithm.

Conclusion We have demonstrated a simple technique for automated i-wave detection that is robust to a slurred type of $\mathrm{i}$-wave. Identification of the preceding concave up turning point as an additional feature is very straightforward as well. These additional features may be useful in the utilisation of statistical learning techniques to ERG traces.

Conflict of interest: None.

Funding: None.

Award applications: Eberhard Dodt Memorial Award.

Corresponding author email: jasha8@gmail.com

\section{P4.3 Comparison of the signal-to-noise ratio between recording electroretinogram with and without pulse reference power line noise reduction}

\section{Tanikawa, A, Shimada, Y, Horiguchi, M}

Department of Ophthalmology, Fujita Health University School of Medicine, Toyoake, Japan

Purpose To compare the signal-to-noise ratio $(\mathrm{S} / \mathrm{N})$ between recording the electroretinogram (ERG) with and without pulse reference power line noise reduction (PURE).

Methods Light adapted (LA) 3.0 ERG was elicited in four eyes of four healthy subjects. The active, reference, and ground electrodes were placed on the lower eyelid, lateral canthus, and earlobe, respectively. The Burian-Allen electrode was placed in the ipsilateral eye. Responses from the skin electrodes and those from the Burian-Allen electrode were recorded in channels 1 and 2, respectively, of the ERG recording equipment (PuREC, Mayo Corp; Inazawa, Japan). The band passfilter was set at a high cut of $500 \mathrm{~Hz}$ and a low cut of $0.3 \mathrm{~Hz}$. Signals were amplified 10,000-fold, and averages were obtained from responses to 64 flashes with an inter-stimulus interval of $1 \mathrm{~s}$. The signal to noise ratio $(\mathrm{S} / \mathrm{N})$ was measured assuming that: 1) The $200 \mathrm{~ms}$ interval (500 ms to $700 \mathrm{~ms}$ ) as far away as possible from the stimulus is the state of noise without ERG response; 2) The root mean square of noise in that interval (RMSn) was expressed by the following formula: $\mathrm{RMSn}=\sqrt{ }\left(1 / \mathrm{n} \sum_{-}(\mathrm{x}=500)^{\wedge} 70(\mathrm{Rx}-\mathrm{Rm})^{\wedge} 2\right)$ where $\mathrm{Rm}$ is the mean value of the potentials in that interval, $\mathrm{Rx}$ is the potential at a certain time, $X$ msec, and $n$ is the total number of data points in that section; 3) Root mean square of the ERG response (RMSs) was calculated between $0 \mathrm{~ms}$ and $200 \mathrm{~ms}$ by 
the same method: $\mathrm{RMSs}=\sqrt{ }\left(1 / \mathrm{n} \sum_{-}(\mathrm{x}=0)^{\wedge} 200(\mathrm{Rx}-\right.$ $\left.\mathrm{Rm})^{\wedge} 2\right)$; 4) $\mathrm{S} / \mathrm{N}$ is expressed by the following formula: $\mathrm{S} / \mathrm{N}$ $(\mathrm{dB})=20 \log _{-} 10 \quad(\mathrm{RMSs} / \mathrm{RMSn})$. The $\mathrm{S} / \mathrm{N}$ was compared between that with and without PURE by paired t-test.

Results The S/N of the Burian-Allen electrode was considerably larger than that of skin electrodes. Both electrodes showed larger $\mathrm{S} / \mathrm{N}$ as the averaging number increased. PURE revealed better $\mathrm{S} / \mathrm{N}$ in skin electrodes, although the differences between the $\mathrm{S} / \mathrm{N}$ with and without PURE was not significant at every averaging number. In the Burian-Allen electrode, the $\mathrm{S} / \mathrm{N}$ with and without PURE were almost identical at any averaging number

Conclusion PURE showed better S/N in LA 3.0 ERG recorded from skin electrodes. Thus, PURE may reduce the averaging number in recording the ERG from skin electrodes.

Conflict of interest: None.

Funding: None.

Award applications: Marmor Award.

Corresponding author email: tani@fujita-hu.ac.jp

\section{P4.4 Quantitative calibration of sensor strip ERG electrodes: Update}

\section{Brodie, S, Chen, D}

Department of Ophthalmology, NYU Langone Health, New York, USA

Purpose ERGs have been traditionally recorded using corneal electrodes, which can be difficult for some patients to tolerate. In the last several years, adhesive skin electrodes have gained in acceptance. We have previously reported on the clinical usefulness of qualitative interpretation of ERG recordings using skin electrodes for a wide spectrum of retinal disorders, as well as a preliminary estimate of the quantitative comparison of simultaneous ERG recordings using contact lens and adhesive skin electrodes to compare the differences in signal strength. In the present report, we update our quantitative findings.

Methods The study was Institutional Review Board approved. 89 subjects who were referred for full-field ERG testing for multiple clinical indications were drawn from the practice of one of the authors (SEB). Informed consent was obtained from patients or their accompanying parent. ERGs (obtained according to ISCEV standards) were recorded simultaneously from both eyes with ERG-jet corneal contact lens electrodes and LKC Technologies Sensor Strip adhesive skin electrodes using multi-channel instrumentation (Diagnosys LLC, Espion3). A-wave and b-wave amplitudes and implicit times were compared between the two electrode types.

Results Waveform morphologies obtained with skin electrodes were similar to those obtained with contact lens electrodes. Regression coefficients (conversion factors) for a-wave and b-wave amplitudes under both photopic and scotopic conditions were tightly clustered: DA 0.01 b-wave: 0.368 ; DA 3.0 a-wave: 0.343 ; DA 3.0 b- wave: 0.360 ; LA 3.0 a-wave: 0.256 ; LA 3.0 b-wave: 0.325 ; $30-\mathrm{Hz}$ flicker peak-to-peak: 0.384 . Regression coefficients for implicit times were nearly equal to 1.0, indicating comparable latencies for recordings from skin electrodes and contact lens electrodes: DA 0.01 b-wave: 0.971 ;
DA 3.0 a-wave: 0.926; DA 3.0 b-wave: 0.996; LA 3.0 a-wave: 0.967; LA 3.0 b-wave: 0.964 . The regression coefficient for the entire amplitude data set was 0.336 , with an overall correlation between skin electrode and contact lens electrode amplitudes of 0.799 . The regression coefficient for the entire implicit time data set was 0.980 , with an overall correlation between skin electrode and contact lens electrode implicit times of 0.96 .

Conclusion Our best estimate for the conversion factor between ERG amplitudes recorded with adhesive skin electrodes and contact lens electrodes is 0.336 (amplitudes with skin electrodes are about $1 / 3$ the amplitudes recorded simultaneously from the same eyes with contact lens electrodes) with a high correlation between skin electrode and contact lens electrode amplitudes. Implicit times are nearly identical for the two electrode types.

Conflict of interest: None.

Funding: None.

Award applications: None.

Corresponding author email: scott.brodie@nyumc.org

\section{P4.5 Full-field scotopic thresholds for color stimuli in patients with retinitis pigmentosa}

Suzuki, Yasutaka ${ }^{1}$, Yang, Lizhu ${ }^{1,2}$, Fujinami-Yokokawa, $\mathbf{Y u}^{1,3,4,5}$, Liu, Xiao ${ }^{1,2,6}$, Farmer, Jeffrey ${ }^{7}$, Tsunoda, Kazushige $^{8}$, Fujinami, Kaoru ${ }^{1,2,5,9}$

${ }^{1}$ Laboratory of Visual Physiology, Division of Vision Research, National Institute of Sensory Organs, National Hospital Organization Tokyo Medical Center, Tokyo, Japan., ${ }^{2}$ Department of Ophthalmology, Keio University School of Medicine, Tokyo, Japan., ${ }^{3}$ Department of Health Policy and Management, Keio University School of Medicine, Tokyo, Japan, ${ }^{4}$ Department of Public Health Research, Yokokawa clinic, Osaka, Japan., ${ }^{5}$ Department of Genetics, UCL Institute of Ophthalmology, London, UK., ${ }^{6}$ Southwest Hospital/Southwest Eye Hospital, Third Military Medical University (Army Medical University), Chongqing, China., ${ }^{7}$ Diagnosys LLC, MA, USA., ${ }^{8}$ Division of Vision Research, National Institute of Sensory Organs, National Hospital Organization Tokyo Medical Center, Tokyo, Japan, ${ }^{9}$ Moorfields Eye Hospital, London, UK

Purpose Full-field stimulus thresholds (FST) are essential in assessing visual function, especially in patients with severe visual impairment. We describe the distribution of luminance thresholds in subjects with retinitis pigmentosa (RP), aiming to establish a deep phenotyping system.

Methods Cases with a clinical diagnosis with RP were enrolled. Comprehensive clinical examinations were performed, including full-field electroretinograms (ffERGs) recorded according to the ISCEV standard. Full-field color stimuli were generated by the Diagnosys Profile ganzfeld ColorDome (Diagnosys, LLC, MA, USA) which utilizes narrow-band LEDs of $448 \mathrm{~nm}$ (blue), $530 \mathrm{~nm}$ (green), and $627 \mathrm{~nm}$ (red). FST was performed according to the previously published method (Klein, Birch 2009). The dark-adapted color FST was performed after $45 \mathrm{~min}$ dark adaptation in the following order: (i) blue; (ii) red; and (iii) white stimulus. A box plot analysis was performed and the cut off value for 1st/ 
3rd quarter was calculated in terms of thresholds for each color FST to assess the distribution. The FST data obtained in RP patients were compared with those of seven healthy participants (median age 29 , range 23 to 45 years old) with no ocular diseases.

Results The median age of disease onset/age at examination of six cases with RP was 12.0 (range, 3 to 40)/62.0 (range, 62 to 72 ). The visual acuity of 12 eyes was very decreased (no light perception in two eyes; light perception in four eyes; hand motion in four eyes; counting finger in two eyes). The full-field ERGs were undetected both in dark-adapted and light-adapted conditions in the six RP cases. The median value of thresholds for blue/red/white FST was 4.97 (range, -7.8 to 13.9 ; 1st to 3 rd quarter cut off value, 3.2 to 6.9 )/8.47 (range, -3.7 to 17.5 ; 1 st to 3 rd quarter cut off value, 6.7 to 10.7 )/11.6 (range, -2.9 to 20.73 ; 1 st to 3 rd quarter cut off value, 2.8 to 11.4$) \mathrm{db}$. The median value of thresholds for blue/red/white FST was $-59.9 /-35.8 /-54.0 \mathrm{db}$ in the seven healthy subjects. FST revealed a significantly different range of thresholds for each colour stimulus between RP patients and healthy subjects.

Conclusion Distribution of luminance thresholds was demonstrated in six cases with RP, which was significantly higher than that of healthy participants. In assessing patients with very severe visual impairment (counting fingers, hand motion, and light perception), quantitative assessment was available using FST, in keeping with previous reports (Roman AJ et al. Physiol Meas. 2007;28(8):N51-6; Klein, Birch Doc Ophthalmol. 2019;119(3):217-224). Further data from additional affected participants are required to validate the clinical investigation in patients with severe visual impairment.

Conflict of interest: All authors have completed Disclosure of Potential Conflicts of Interest. Individual investigators who participate in the sponsored project(s) are not directly compensated by the sponsor but may receive salary or other support from the institution to support their effort on the project(s). Kaoru Fujinami is a paid consultant of Astellas Pharma Inc, Kubota Pharmaceutical Holdings Co; Ltd, Acucela Inc; Janssen Pharm, Sanofi Genzyme, and NightstaRx Limited. Kaoru Fujinami reports personal fees from Astellas Pharma Inc, personal fees from Kubota Pharmaceutical Holdings Co; Ltd; personal fees from Acucela Inc; personal fees from NightstaRx Limited; personal fees from SANTEN Company Limited, personal fees from Foundation Fighting Blindness, personal fees from Foundation Fighting Blindness Clinical Research Institute, personal fees from Japanese Ophthalmology Society, personal fees from Japan Retinitis Pigmentosa Society. Laboratory of Visual Physiology, Division for Vision Research, National Institute of Sensory Organs, National Hospital Organization, Tokyo Medical Center, Tokyo, Japan is supported by grants from Astellas Pharma Inc (NCT03281005), outside the submitted work. Jeffrey Farmer is the CEO of Diagnosys LLC.

Funding: Yu Fujinami-Yokokawa was supported by grants from Grant-in-Aid for Young Scientists of the Ministry of Education, Culture, Sports, Science and Technology, Japan (18K16943). Kaoru Fujinami is supported by grants from Grant-in-Aid for Young Scientists (A) of the Ministry of Education, Culture, Sports, Science and Technology, Japan (16H06269), grants from Grant-in-Aid for Scientists to support international collaborative studies of the Ministry of Education, Culture, Sports, Science and Technology, Japan
(16KK01930002), grants from National Hospital Organization Network Research Fund (H30-NHO-Sensory Organs-03), grants from FOUNDATION FIGHTING BLINDNESS ALAN LATIES CAREER DEVELOPMENT PROGRAM (CF-CL0416-0696-UCL), grants from Health Labour Sciences Research Grant, The Ministry of Health Labour and Welfare (201711107A), and grants from Great Britain Sasakawa Foundation Butterfield Awards. Role of the Funder/Sponsor: The funding sources had no role in the design and conduct of the study; collection, management, analysis, and interpretation of the data; preparation, review, or approval of the manuscript; and decision to submit the manuscript for publication.

Award applications: Eberhard Dodt Memorial Award.

Corresponding author email: k.fujinami@ucl.ac.uk

\section{Poster Session 5: Genotype-Phentype Correlations}

\section{P5.1 Correlation of electrophysiological parameters and genotype of ABCA4-associated retinal disease}

Zolnikova, $\mathbf{I}^{1}$, Kadyshev, $\mathbf{V}^{2}$, Marakhonov, $\mathrm{A}^{3}$

${ }^{1}$ Department of Clinical Physiology of Vision after Kravkov, Helmholtz National Medical Research Center of Eye Diseases, Moscow, Russian Federation; ${ }^{2}$ Laboratory of Genetic Epidemiology, Research Centre for Medical Genetics, Moscow, Russian Federation; ${ }^{3}$ Laboratory of Genetic Epidemiology, Research Centre for Medical Genetics, Russian Federation

Purpose To study genotype-phenotype correlations in patients with inherited retinal diseases with mutations in ABCA4 gene in the Russian Federation.

Methods 21 patients from the Russian population aged 7 to 51 years old (mean age $20 \pm 11$ years) with best-corrected visual acuity from 0.02 to $0.6(0.14 \pm 0.11)$ with ABCA4associated retinopathy verified by molecular genetics methods were evaluated. All patients underwent standard ophthalmic examination and photodocumentation using Spectral-Domain OCT and fundus autofluorescence on Spectralis ${ }^{\circledR} \mathrm{HRA}+\mathrm{OCT}$ (Heidelberg Engineering, Germany). The full-field electroretinogram (ERG), 30-Hz flicker ERG, and macular chromatic ERG (MERG) to red stimuli were recorded on the electroretinographic system (MBN, Russia). Molecular genetic studies were performed using Next Generation Sequencing (NGS) and Sanger direct sequencing.

Results In ABCA4-associated Stargardt disease (STRG1) genotype [p.L541P, p.A1038V], mutations were found in nine patients. In two cases it was associated with another frequent mutation p.G1961E. In four patients with genotype [p.L541P, p.A1038V], a severe phenotype of Stargardt disease was found showing large defects of the ellipsoid zone, a large zone of central reduced autofluorescence, severely subnormal macular ERG (MERG) to red stimulus and subnormal $30 \mathrm{~Hz}$ flicker and full-field maximal ERG. In one patient with these mutations in the homozygous state ABCA4-associated cone-rod dystrophy (CRD), clinically looking like retinal dystrophy, was diagnosed. In two patients with genotype [p.L541P, p.A1038V] and mutation p.G1961E, the phenotype was mild. The other 18 
mutations were: 11 missense (p.R653C, p.C1488R, p.Q635X, p.E471K, p.R2106C, p.N1868I, p.R212C, p.L210Q, p.His1838Arg, p.Arg653Cys, p.R653C), three nonsense (p.Q1412*,p.Arg2201Alafs*, p.Tyr1947*), one deletion (p.R124fs), and two splicing site mutations (c.2588-12C > G and c. $1937+1 \mathrm{G}>\mathrm{A}$ ). Nonrecordable MERG was found in seven of 21 patients $(1 / 3)$; the rest were subnormal. Subnormal $30 \mathrm{~Hz}$ flicker ERG was found in eight patients with SD and one patient with CRD. Maximal ERG was reduced in six patients with Stargardt Disease and one patient with CRD. One patient with homozygous mutation p.R653C autosomal recessive ABCA4-associated retinitis pigmentosa (RP 19) was diagnosed and had nonrecordable MERG, 30-Hz flicker and Maximal ERG. OCT demonstrated an ellipsoid zone defect of differing extent in all patients. The clinical picture and autofluorescence were polymorphic in all patients.

Conclusion Our study using ophthalmological, molecular genetics and instrumental methods widens the spectrum of clinical signs of inherited eye diseases associated with mutations in the ABCA4 gene, widens the spectrum of mutations found in the Russian Federation, and reveals clinico-genetic correlations.

Conflict of interest: None.

Funding: Russian Scientific Fund (project No. 17-15-01051). Award applications: None.

Corresponding author email: innzolnikova@hotmail.com

\section{P5.2 Electrophysiological findings of patients with RPGR-associated retinal disorder}

Nakamura, $\mathbf{N}^{1,2}$, Fujinami, $\mathbf{K}^{1,3,4,5}$, Naoi, $\mathbf{N}^{6,7}$, Ikeda, $\mathbf{Y}^{6}$, Hayashi, $\mathbf{T}^{\mathbf{8}}$, Ueno, $\mathbf{S}^{\mathbf{9}}$, Terasaki, $\mathbf{H}^{\mathbf{9}}$, Kuniyoshi, $\mathbf{K}^{10}$ ,Mizota, $A^{11}$, Shinoda ${ }^{11,12}$, Yang, $L^{1,3}$, FujinamiYokokawa, $\mathbf{Y}^{1,4,13,14}$, Liu, $\mathbf{X}^{1,3,15}$, Kominami, $T^{9}$, Komori, $\mathbf{S}^{\mathbf{9}}$, Sakuramoto, $\mathbf{H}^{10}$, Katagiri, $\mathbf{S}^{\mathbf{8}}$, Mizobuchi, $\mathbf{K}^{\mathbf{8}}$, Mawatari, $G^{6}$, Miyake, $Y^{1,16,17}$, Yoshitake, $K^{18}$, Iwata, $T^{18}$ , Tsunoda, $\mathbf{K}^{1}$, JEGC study group

${ }^{1}$ Laboratory of Visual Physiology, Division of Vision Research, National Institute of Sensory Organs, National Hospital Organization Tokyo Medical Center, Tokyo, 152-8902, Japan, ${ }^{2}$ Department of Ophthalmology, The University of Tokyo, Tokyo, 113-8655, Japan, ${ }^{3}$ Department of Ophthalmology, Keio University School of Medicine, Tokyo, 160-8582, Japan, ${ }^{4}$ UCL Institute of Ophthalmology, London, EC1V 9EL, UK, ${ }^{5}$ Moorfields Eye Hospital, London, EC1V 2PD, UK, ${ }^{6}$ Department of Ophthalmology, Miyazaki University, Miyazaki, Miyazaki, 889-1692, Japan, ${ }^{7}$ Miyazaki Chuoh Eye Hospital, Miyazaki, Miyazaki, 880-0021, Japan, ${ }^{8}$ Department of Ophthalmology, The Jikei University School of Medicine, Tokyo, 105-8471, Japan, ${ }^{9}$ Department of Ophthalmology, Nagoya University Graduate School of Medicine, Nagoya, Aichi, 466-8550, Japan, ${ }^{10}$ Department of Ophthalmology, Kindai University Faculty of Medicine, Faculty of Medicine, Osaka-Sayama, Osaka, 589-8511, Japan, ${ }^{11}$ Department of Ophthalmology, Teikyo University, Tokyo, 173-8605, Japan, ${ }^{12}$ Department of Ophthalmology, Saitama Medical University, Moroyama, Saitama, 350-0495, Japan, ${ }^{13}$ Department of Health Policy and Management, Keio University School of
Medicine, 160-8582, Tokyo, Japan, ${ }^{14}$ Division of Public Health, Yokokawa Clinic, Suita, 564-0083 Japan, ${ }^{15}$ Southwest Hospital/Southwest Eye Hospital, Third Military Medical University (Army Medical University), Chongqing, China, ${ }^{16}$ Aichi Medical University, Nagakute, Aichi, 480-1195, Japan, ${ }^{17}$ Next Vision, Kobe Eye Center, Hyogo, 650-0047, Japan, ${ }^{18}$ Division of Molecular and Cellular Biology, National Institute of Sensory Organs, National Hospital Organization Tokyo Medical Center, Tokyo, 152- 8902 Japan

Purpose Pathogenic variants in the retinitis pigmentosa GTPase regulator (RPGR; MIM: 312610) gene cause a vast range of phenotypes with an X-linked inheritance: rod-cone dystrophy (RCOD), cone-rod dystrophy (CORD), cone dystrophy (COD), macular dystrophy (MD), and non-ocular phenotypes (Tee JJ, Br J Ophthalmol. 2016;100:1022-7). We describe the spectrum of electrophysiological findings of RPGR-associated retinal diseases (RPGR-RD) in a Japanese nationwide cohort.

Methods Twenty-one affected males with a clinical diagnosis with inherited retinal disease and harboring pathogenic RPGR variants were selected via the database of the Japan Eye Genetics Consortium (JEGC; 2209 subjects from 1277 families), and 15 patients including one sibship with available ERG data were included in this study. Some of the data have been partially published previously (Mawatari G et al. Hum Gen Var 2018;6:34; Kurata et al. Int J Mol Sci. 2019;20(6):1518). The registered data of comprehensive clinical examinations were reviewed, including full-field electroretinograms (ffERGs) and multifocal electroretinograms (mfERGs) recorded with the ISCEV standard protocols. Patients were classified into one of the four functional phenotypes mainly based on the fundus, ffERG, and mfERG findings; RCOD, CORD, COD, and MD. Clinical parameters were compared among the functional phenotypes. An association between the functional phenotypes and the positions of variants (cut off of amino acid 950) was investigated.

Results The median age of disease onset/age at examination of 15 patients was, 41.0 (range, 12 to 72 ) years/8.0 (3 to 50) years. The median visual acuity was $0.30(-0.08$ to 1.70$) / 0.22(-0.18$ to 1.52 ) $\log$ MAR in the right/left eye, respectively. There were nine patients $(9 / 15,60 \%)$ in RCOD functional phenotype, six $(6 / 15,40 \%)$ patients in CORD functional phenotype. No patients were in COD or MD phenotypes. There was a trend of difference between RCOD and CORD in terms of age of onset, with the median value being 5.0 years for RCOD and 15.0 years for CORD ( $p=0.07$, Mann-Whitney U test). There was no significant difference regarding the visual acuity or the refractive error (spherical equivalent). All RPGR variants in RCOD were located on the $5^{\prime}$ side and all variants in CORD were on the $3^{\prime}$ side, which revealed a statistically significant association between the functional phenotypes and the positions of variants ( $p<0.01$, Fisher's Exact test).

Conclusion Two functional phenotypes of RCOD and CORD were identified in a large Japanese cohort with RPGR-RD. The early-onset disease with primal generalized rod dysfunction and the later-onset disease with primal cone dysfunction were associated with the genetic characteristics.

Conflict of interest: All authors have completed Disclosure of Potential Conflicts of Interest. Individual investigators who 
participate in the sponsored project(s) are not directly compensated by the sponsor but may receive salary or other support from the institution to support their effort on the project(s). Kaoru Fujinami is a paid consultant of Astellas Pharma Inc, Kubota Pharmaceutical Holdings Co; Ltd, Acucela Inc; Janssen Pharm, Sanofi Genzyme, and NightstaRx Limited. Kaoru Fujinami reports personal fees from Astellas Pharma Inc, personal fees from Kubota Pharmaceutical Holdings Co; Ltd; personal fees from Acucela Inc; personal fees from NightstaRx Limited; personal fees from SANTEN Company Limited, personal fees from Foundation Fighting Blindness, personal fees from Foundation Fighting Blindness Clinical Research Institute, personal fees from Japanese Ophthalmology Society, personal fees from Japan Retinitis Pigmentosa Society. Laboratory of Visual Physiology, Division for Vision Research, National Institute of Sensory Organs, National Hospital Organization, Tokyo Medical Center, Tokyo, Japan is supported by grants from Astellas Pharma Inc (NCT03281005), outside the submitted work.

Funding: Yu Fujinami-Yokokawa was supported by grants from Grant-in-Aid for Young Scientists of the Ministry of Education, Culture, Sports, Science and Technology, Japan (18K16943). Kaoru Fujinami is supported by grants from Grant-in-Aid for Young Scientists (A) of the Ministry of Education, Culture, Sports, Science and Technology, Japan (16H06269), grants from Grant-in-Aid for Scientists to support international collaborative studies of the Ministry of Education, Culture, Sports, Science and Technology, Japan (16KK01930002), grants from National Hospital Organization Network Research Fund (H30-NHO-Sensory Organs-03), grants from FOUNDATION FIGHTING BLINDNESS ALAN LATIES CAREER DEVELOPMENT PROGRAM (CF-CL0416-0696-UCL), grants from Health Labour Sciences Research Grant, The Ministry of Health Labour and Welfare (201711107A), and grants from Great Britain Sasakawa Foundation Butterfield Awards. Role of the Funder/Sponsor: The funding sources had no role in the design and conduct of the study; collection, management, analysis, and interpretation of the data; preparation, review, or approval of the manuscript; and decision to submit the manuscript for publication.

Award applications:

Corresponding author email: k.fujinami@ucl.ac.uk

\section{P5.3 Autosomal dominant adult vitelliform dystrophy secondary to IMPG2 mutation}

\section{Lee, CS, Odom, JV, Salido, E, Leys, M}

Ophthalmology, West Virginia University, Morgantown, WV, USA

Purpose To illustrate the use of the EOG to distinguish retinal diseases characterized by foveal vitelliform lesions.

Methods A 65-year-old Caucasian male with maculopathy was referred for electrooculogram (EOG) and multimodal imaging. $\mathrm{He}$ was a non-smoker with diabetes. Initial best-corrected visual acuity (BCVA) was $20 / 50$ in the right $(+0.50-$ $1.75 \times 097)$ and $20 / 60$ in the left $(+1.00-1.00 \times 087)$.

Results Fundus examination showed bilateral vitelliform lesions and moderate non-proliferative diabetic retinopathy.
Subfoveal vitelliform deposits without neovascular complexes were seen on optical coherence angiography (OCTA). Fundus autofluorescence (FAF) demonstrated a patchy heterogeneous pattern at the fovea (hypo-autofluorescent in the right, hyperautofluorescent in the left). EOG showed Arden ratio of 2.45 and 3.41 in the right and the left eye, respectively. Genetic mutation screening was performed ( 248 gene NGS IRD panel Spark Initiative Invitae), and a heterozygous mutation was found in the IMPG2 gene: c.2890C > T (p.Arg964*). Another mutation was found in BEST1 gene: c.584C $>\mathrm{T}$ (p.Ala195Val). However, his presentation was most consistent with autosomal dominant adult vitelliform dystrophy secondary to IMPG2 mutation, given the normal EOG. He was observed for 3 years without any treatment, and there was no progression of the vitelliform lesions. His BCVA was maintained at 20/40 bilaterally.

Conclusion This case highlights that patients with foveal vitelliform lesions may be tested with EOG to make an initial determination of whether the disease is consistent with Best vitelliform macular dystrophy. As the FAF imaging pattern of Best disease can be variable, genetic testing is desirable to differentiate it from other causes of vitelliform macular dystrophy (Parodi et al., Graefes Arch Clin Exp Ophthalmol. 2016;254(7):1297-1302). In our case, the patient had a mutation in the gene IMPG2 related to the photoreceptor extracellular matrix proteoglycan (Salido et al., J Neurosci. 2020;40(20):4059-4072). This gene is not linked to the development of Best disease, and patients with mutations in this gene may present as adult onset foveomacular vitelliform dystrophy with normal EOG findings (Meunier et al., Ophthalmology. 2014;121(12):2406-2414). These patients can be monitored without any treatment.

Conflict of interest: None.

Funding: None.

Award applications: None.

Corresponding author email: changsupleemd@gmail.com

P5.4 Electrophysiological findings in Wolfram Syndrome (WFS1), associated with optic neuropathy

Neveu, $\mathbf{M}^{1,2}$, Huang, $\mathbf{H B}^{3}$, Majander, $\mathrm{Aa}^{1,2,4}$, Jurkute, $\mathbf{N}^{1,2}$, Arno, $\mathbf{G}^{1,2}$, Webster, $\mathbf{A}^{1,2}$, Michaelides, $\mathbf{M}^{1,2}$, Y-WaiMan, $\mathbf{P}^{1,2,5,6}$, Robson, $A^{1,2}$

${ }^{1}$ UCL Institute of Ophthalmology, London, UK; ${ }^{2}$ Moorfields Eye Hospital, London, UK; ${ }^{3}$ Hainan Hospital of the General Hospital of Chinese People's Liberation Army, Sanya, China; ${ }^{4}$ Department of Ophthalmology, Helsinki University Hospital, and University of Helsinki, Helsinki, Finland; ${ }^{5}$ Medical Research Council Mitochondrial Biology Unit, Cambridge, UK; ${ }^{6}$ Department of Clinical Neurosciences, School of Clinical Medicine, University of Cambridge, Cambridge, UK

Purpose To describe the electrophysiological findings in twelve patients with inherited optic neuropathy (ION) caused by variants in WFS1 gene associated with Wolfram syndrome (AR inheritance) or Wolfram-like syndrome (AD inheritance). Electrophysiological parameters were compared between the 
autosomal recessive (AR) WFS1 and autosomal dominant (AD) WFS1 patient groups.

Methods Twelve patients with confirmed molecular diagnosis of Wolfram or Wolfram-like syndrome (WSF1), aged 7 to 57 years (median 23 years) were examined in the Electrophysiology Department at Moorfields Eye Hospital. All patients underwent electrophysiological testing including ISCEV standard pattern and flash visual evoked potential (PVEP; FVEP) and pattern and full-field electroretinogram (PERG; ERG). In addition, the photopic negative response (phNR) was recorded in nine patients.

Results Four of 12 patients (three families) were carrying biallelic pathogenic variants in WFS1 and eight (four families) were heterozygous for a dominant pathogenic allele causing the disease. Twenty eyes had an undetectable or a delayed PVEP; five eyes had amplitude reduction in addition to delay. Seventeen eyes had normal FVEPs; five eyes had subnormal FVEPs and two eyes had subnormal and delayed FVEPs. PVEPs were similar in both patient groups, but the FVEP was more delayed in the AD WFS1 group only (Mann-Whitney U Test, $p<0.05)$. The PERG N95:P50 amplitude ratio was abnormal in nine patients and normal in three; PERG P50 amplitude was normal or of borderline amplitude in both eyes of 10 patients and subnormal in one patient. PERG P50 peak time was abnormally short in all patients with AR WFS1 and in three eyes of two patients with AD WFS1. Nine of 12 patients who underwent standard full-field ERG testing showed no clinically significant abnormality. The phNR/b-wave ratios was reduced in only one eye of one patient.

Conclusion This study is the first to describe PERG and phNR abnormalities in WFS1- related optic neuropathy. The majority of patients showed reduction of the PERG N95:P50 ratio and relative preservation of the phNR, in keeping with central rather than peripheral retinal ganglion cell (RGC) involvement. The findings are consistent with RGC dysfunction being more severe in autosomal recessive than in autosomal dominant cases of WFS1-related optic neuropathy.

Conflict of interest: None.

Funding: None.

Award applications: None.

Corresponding author email: Magella.Neveu@nhs.net

P5.5 Next-generation sequencing identified three novel variants in REEP6 gene in three retinitis pigmentosa families with severely decreased ERG responses

Zhang, Lujia ${ }^{1}$, Ya, $\mathbf{L i}^{2}$, Chen, Kang ${ }^{3}$, Guo, Xiaohong ${ }^{3}$, Sui, Ruifang ${ }^{4}$, Lei, Bo ${ }^{5}$

${ }^{1}$ Xinxiang Medical University, ${ }^{2}$ Henan Provincial People's Hospital, Henan Eye Institute, Henan Eye Hospital, Henan Clinical Research Center for Ophthalmic Diseases, ${ }^{3}$ Henan Provincial People's Hospital, Henan Eye Hospital, ${ }^{4}$ Department of Ophthalmology,Peking Union Medical College Hospital,Peking Union Medical College, Chinese Academy of Medical Sciences, ${ }^{5}$ Henan Provincial People's Hospital, People's Hospital of Zhengzhou University, Henan Eye Institute, Henan Eye Hospital, Henan Clinical Research Center for Ophthalmic Diseases
Purpose The purpose of this study was to identify the underlying gene defect leading to retinitis pigmentosa in three Chinese families with severely decreased ERG responses.

Methods Three Chinese male probands from three different families complained of night blindness and decreased visual acuity. Ophthalmic examinations including fundus photography, spectral domain optical coherence tomography (SDOCT), fundus autofluorescence (AF), infrared reflectance (IR) and full-field electroretinogram (ERG) were conducted. Blood samples from probands and their families were collected. Nextgeneration sequencing followed by Sanger validation was performed. Bioinformatics and homogeneous analysis and protein structure prediction were performed.

Results All three probands had tunnel visual fields and poor visual acuity. The fundus showed bone-spicule deposits. The ERG responses were non-recordable in one proband, while all ERG responses were severely decreased in the other two probands. Outer nuclear layer and ellipsoid zone loss outside the fovea was observed in one proband with evident bilateral macular hole $(\mathrm{MH})$ and epiretinal membranes (ERM). Nextgeneration sequencing identified three novel variants in REEP6, including one missense variant in exome 3 of REEP6 (c.268G > C, p. V90L, NM_138393), one frameshift deletion variant in exome 4 of REEP6 (c.468del C, p. A156fs*14, NM_138393) and one splicing variant in intron 4 of REEP6 (c. $517+1 G>C$, NM_138393). Bioinformatics analysis with Mutation Taster, SIFT and PolyPhen2 indicated the variants were harmful. Based on the prediction of protein structure, the receptor expression enhancing protein 6 encoded by the variants may be changed, which was involved in the transport of receptors from the endoplasmic reticulum (ER) to the cell surface.

Conclusion Three novel variants in REEP6 may be diseasecausing in three retinitis pigmentosa families with severely decreased ERG responses. What we found expands the mutation spectrum of REEP6, as only nine variants leading to retinitis pigmentosa have been reported worldwide.

Conflict of interest: None.

Funding: National Natural Science Foundation of China (81770949).

Award applications: Eberhard Dodt Memorial Award. Corresponding author email: bolei99@126.com

\section{Poster Session 6: Assessing Retinal Function in Inherited Retinopathies}

P6.1 Oguchi disease caused by a homozygous novel SAG splicing alteration associated with the multiple evanescent white dot syndrome: A 15-month follow-up

Liu, $X^{1,2,3,4}$, Gao, $L^{1,2}$, Yang, $L^{3,4}$, Fujinami-Yokokawa, $Y^{3,5,6,7}$, Wang, $G^{1,2}$,Long, $Y^{1,2}$, Ren, $\mathbf{J}^{1,2}$, Fujinami, $\mathbf{K}^{3,4,7,8}$, Meng, $X^{1,9}, \mathrm{Li}, S^{1,2}$

${ }^{1}$ Southwest Hospital/Southwest Eye Hospital, Third Military Medical University (Army Medical University), Chongqing, China., ${ }^{2}$ Key Lab of Visual Damage and Regeneration \& Restoration of Chongqing, Chongqing, China., ${ }^{3}$ Laboratory of Visual Physiology, Division for 
Vision Research, National Institute of Sensory Organs, National Hospital Organization, Tokyo Medical Center, Tokyo, Japan., ${ }^{4}$ Department of Ophthalmology, Keio University School of Medicine, Tokyo, Japan, ${ }^{5}$ Department of Health Policy and Management, Keio University School of Medicine, Tokyo, Japan., ${ }^{6}$ Division of Public Health, Yokokawa Clinic, Suita, Osaka, Japan., ${ }^{7}$ Department of Genetics, UCL Institute of Ophthalmology, London, UK, ${ }^{8}$ Division of Inherited Eye Diseases, Moorfields Eye Hospital, London, UK, ${ }^{9}$ Key Lab of Visual Damage and Regeneration \& Restoration of Chongqing, Chongqing, China

Purpose We report a 15-month follow-up case on a Chinese patient with Oguchi disease associated with the multiple evanescent white dot syndrome (MEWDS).

Methods The patient's clinical presentation and follow-up visits were documented via decimal best-corrected visual acuity, fundus photography, fundus autofluorescence (FAF) imaging, near-infrared FAF, spectral-domain optical coherence tomography, Humphrey visual fields, microperimetry, and multifocal electroretinography. We also performed wholeexome sequencing for screening variation in the patient and her relatives.

Results The 28-year old female had a typical clinical phenotype of Oguchi disease, including night blindness, the Mizuo-Nakamura phenomenon [a golden yellow discoloration of the fundus that disappears with prolonged dark adaptation (DA)] and typical full-field electroretinogram changes (barely detectable b-wave in DA 0.01 ERG and severely reduced aand b-wave in DA 3.0 ERGs that partially recovers only after prolonged DA). Aside from Oguchi disease, the patient was also diagnosed with MEWDS based on the clinical findings, including suddenly reduced visual acuity, appearance of white dots, blurred ellipsoid zone, disrupted interdigitation zone, enlarged blind spot, and reduced macular sensitivity. A series of investigations during the 15-month follow-up after onset revealed that the visual acuity improved, the numerous white dots disappeared, and the macular structure returned to normal. Moreover, a novel splice site alteration $(\mathrm{c} .181+1 \mathrm{G}>\mathrm{A})$ in the homozygous status was identified in the SAG gene.

Conclusion This is the first long-term case study of a patient with Oguchi disease associated with MEWDS. The recovery period of symptoms caused by MEWDS was much longer than that in typical patients with MEWDS. Molecular genetics demonstrates that this is the first case of Oguchi disease caused by splicing alterations in the SAG gene. A relationship between Oguchi disease and MEWDS is uncertain; however, it is of note that the recovery course for MEWDS was prolonged.

Conflict of interest: None.

Funding: National Nature Science Foundation of China (81974138).

Award applications: Eberhard Dodt Memorial Award, Marmor Award.

Corresponding author email: shiying_li@126.com
P6.2 Sine-wave flicker ERG reflecting postreceptoral activation in controls and Duchenne muscular dystrophy (DMD) patients

Barboni, Mirella TS ${ }^{1}$, Silva, Leonardo $\mathrm{A}^{2}$, Dias, Sarah $\mathbf{L}^{2}$, Damico, Francisco $\mathbf{M}^{2}$, Vidal, Kallene $\mathrm{S}^{2}$, Costa, Marcelo $F^{2}$, Ventura, Dora $F^{2}$, Kremers, $\operatorname{Jan}^{3}$

${ }^{1}$ Department of Ophthalmology, Semmelweis University, Budapest, Hungary; ${ }^{2}$ Experimental Psychology, University of Sao Paulo, Sao Paulo, Brazil; ${ }^{3}$ Department of Ophthalmology, University Hospital Erlangen, Erlangen, Germany

Purpose In healthy humans, the first harmonic component of ERGs to sine-wave luminance modulation displays a minimal amplitude at around $12 \mathrm{~Hz}$, while at 30 to $40 \mathrm{~Hz}$, a maximum is observed. It has been proposed that two independent components give rise to these ERG responses: the 'sinusoidal' component is particularly large at low temporal frequency whereas the 'transient' component determines the responses at high temporal frequencies (Pangeni et al., Vis Neurosci 2010;27:70-90). It has been further proposed that at $12 \mathrm{~Hz}$, on- and off-signals may cancel each other (Kondo \& Sieving, IOVS 2001;42:305-312). Here we examine whether the abnormal post-receptoral on-off balance, as found in patients with Duchenne muscular dystrophy (DMD), could asymmetrically affect the sine-wave flicker ERG components and thus influence the sinusoidal or the transient components or both. Methods ISCEV standard full-field ERG and ERG responses to photopic sawtooth rapid-on/rapid-off and sine-wave flicker $(12$ and $36 \mathrm{~Hz})$ were recorded in 14 DMD patients and 14 agematched healthy volunteers. In the patient group, 10 subjects (DMD down30) had a genetic alteration affecting the expression of Dp260 in addition to the full-length dystrophin Dp427, and four subjects had a genetic alteration affecting only the expression of Dp427. ISCEV standard a- and b-waves and sawtooth negative/positive components were analyzed in the time domain. Sine-wave flicker responses were analyzed in the frequency domain [after fast Fourier transform (FFT)] to extract first harmonic amplitudes and phases and using a previously described procedure (Pangeni et al., $2010 \mathrm{ibid}$ ) to extract the sinusoidal and the transient components.

Results ERG showed significantly lower $(p<0.001)$ b/a and on/off ratios in DMD down30 patients because of reduced b-wave amplitude $(p=0.003)$ with normal a-waves $(p=0.812)$ and reduced amplitude on-responses $(p=0.047)$ with normal off-responses $(p=0.319)$, respectively. The first harmonic component to $12 \mathrm{~Hz}$ sine-wave stimuli had larger amplitudes $(p<0.001)$ and were phase shifted $(p=0.020)$ in DMD down30 patients compared to controls, but they were similar to controls at $36 \mathrm{~Hz}$ (amplitude $p=0.205$ and phase $p=0.999$ ). The $12 \mathrm{~Hz}$ sinusoidal component was specifically affected $(p=0.018)$ in DMD down30 patients as the $12 \mathrm{~Hz}$ transient component was similar in DMD patients and controls $(p=0.683)$. A positive correlation was found between the 
amplitudes of the first harmonic and the sinusoidal components in DMD patients (Spearman 0.893, $p<0.001$ ), but not in controls (Spearman 0.386, $p=0.216$ ).

Conclusion Reduced b/a ratios and reduced on-responses in DMD down30 patients reflect an abnormal photoreceptor to ON bipolar cell signal transmission caused by Dp260 dysfunction. The resulting unbalanced ON/OFF bipolar cell activation or interaction may exaggerate the contribution of the sinusoidal relative to the transient component in the ERG to sine-wave luminance modulation.

Conflict of interest: None.

Funding: This study was supported by the Sao Paulo Research Foundation (FAPESP grant numbers 2016/04538-3 and $2014 / 26818-2$ to DFV, and 2017/16948-4 to LAS), the National Council for Scientific and Technological Development (CNPq grant number 404239/2016-1 to MTSB), DAAD in Germany and Tempus Public Foundation in Hungary to JK and MTSB, and a CNPq 1A productivity fellowship to DFV. Award applications: None.

Corresponding author email: mtsbarboni@gmail.com

P6.3 The clinical and electrophysiological features associated with RLBP1-retinopathy

Duignan, Emma ${ }^{1}$, Schiff, Elena ${ }^{1}$, Wright, Genevieve ${ }^{1}$, Knight, Hannah ${ }^{1}$, Michaelides, Michel ${ }^{1,2}$, Webster, Andrew ${ }^{1,2}$, Robson, Anthony G ${ }^{1,2}$

\author{
${ }^{1}$ Moorfields Eye Hospital, London, UK; ${ }^{2}$ University \\ College London, London, UK
}

Purpose To examine the clinical and electrophysiological phenotype associated with bi-allelic variants in RLBP1.

Methods Patients were identified through the Moorfields Eye Hospital electronic healthcare records. The results of fundus imaging including fundus autofluorescence (FAF) were reviewed. Nine patients underwent ISCEV standard pattern and full-field electroretinography (PERG; ERG); the ERGs were additionally recorded after overnight dark adaptation in most. Leukocyte genomic DNA was extracted and genetic sequencing was carried out by panel-based testing, whole exome, or genome sequencing.

Results Eleven patients (age range 9-87 years, 8 females) were identified to have bi-allelic RLBP1 variants. Visual acuity ranged from $6 / 6$ to NPL. At the last visit, all patients under the age of 30 years maintained a best-corrected visual acuity (BCVA) of at least 6/9 (excluding one amblyopic eye with BCVA of 6/12). The most common findings on fundus examination were peripheral fine white dots at the level of the RPE $(n=6)$ and/or pigmentary disturbance $(n=8)$. All patients with FAF imaging showed diffusely reduced AF. OCT revealed foveal outer retinal atrophy with parafoveal sparing in two patients. Pattern ERG P50 components were subnormal in six patients including four with undetectable responses. Fullfield ERGs were undetectable ( $\mathrm{n}=1$; aged 70 years), consistent with rod-cone dystrophy $(n=7)$ or normal $(n=1$; age 9 years). Following overnight dark adaptation, the strong flash (DA10) ERGs remained undetectable in one patient; there was ERG a-wave enlargement (mean increase 80\%) and b-wave peak time increase in four others, in keeping with an increased rod system contribution. Five novel RLBP1 mutations were identified, two homozygously, c.701G > A, p.Arg234Gln and c. $525+802 \_3388 \mathrm{del}$, and three in compound heterozygote states, $\quad$ IVS3 +2 delT, c. $12+2$ del and c. $304 \mathrm{G}>\mathrm{A}$, p.Ala102Thr.

Conclusion RLBP1-retinopathy can be variable, with white dots, pigmentary abnormalities and reduced autofluorescent signal representing the most common features. Full-field ERGs indicate rod-cone dystrophy in most, with limited recovery of rod function following overnight dark adaptation. This is the first case of RLBP1-retinopathy with a normal full-field ERG reported to date and suggests a window of opportunity for therapeutic intervention.

Conflict of interest: None.

Funding: None.

Award applications: Eberhard Dodt Memorial Award.

Corresponding author email: emmaduignan@gmail.com

P6.4 MERTK retinopathy: understanding rate of visual loss in the therapeutic era

Sakti, Dhimas Hari ${ }^{1,2,3,4,5,6,7,8,9,10,11}$, Cornish,

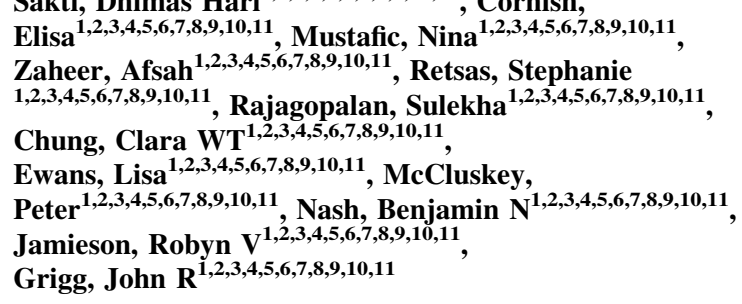

${ }^{1}$ Save Sight Institute, Faculty of Medicine and Health, The University of Sydney, Sydney, NSW, Australia; ${ }^{2}$ Department of Ophthalmology, Faculty of Medicine, Public Health and Nursing, Universitas Gadjah Mada, Yogyakarta, Indonesia; ${ }^{3}$ Eye Genetics Research Unit, Children's Medical Research Institute, The Children's Hospital at Westmead, Save Sight Institute, University of Sydney, Sydney, NSW, Australia; ${ }^{4}$ Department of Clinical Genetics, Liverpool Hospital, Locked Bag 7103, Liverpool BC, NSW 1871, Australia; ${ }^{5}$ School of Women's \& Children's Health, University of NSW, Sydney, Australia; ${ }^{6}$ Department of Clinical Genetics Royal Prince Alfred Hospital Camperdown NSW, Australia; ${ }^{7}$ Faculty of Medicine and Health Central Clinical School, Sydney Medical School, University of Sydney, Sydney, NSW, Australia; ${ }^{8}$ Disciplines of Genomic Medicine \& Child and Adolescent Health, Sydney Medical School, University of Sydney, NSW, Australia, ${ }^{9}$ Sydney Genome Diagnostics, Western Sydney Genetics Program, The Children's Hospital at Westmead, Sydney, NSW, Australia;

${ }^{10}$ Department of Clinical Genetics, Western Sydney Genetics Program, The Children's Hospital at Westmead, Sydney, NSW, Australia

Purpose Mutated MER Tyrosine Kinase (MERTK) is expressed in the retinal pigment epithelium (RPE) of retinitis pigmentosa (RP) patients. Understanding the rate of visual loss in this rare group of patients will guide the optimum time for therapeutic intervention. 
Methods Retrospective patient data cohorts from baseline (BL) and last follow-up (LFU) were reviewed. Best corrected visual acuity (BCVA), spectral domain-optical coherence tomography (SD-OCT) parameters including ellipsoid zone (EZ) length and central macular thickness (CMT), ultra-widefield fundus autofluorescence (UWF-FAF) patterns, semi-automated kinetic perimetry (SKP), and electroretinography (ERG) parameters were investigated.

Results Five patients were included in with mean age at BL diagnosis of 20.9 years (14.4-42.3 years). Mean follow up was 51.4 months (22.3-61.1 months). Mean BCVA at BL and LFU was $0.87 \pm 0.84 \quad(0.30-2.40) \quad \log$ MAR and $1.09 \pm 0.92$ (0.32-2.70) $\log$ MAR, respectively, with decline rate of 0.07 $\operatorname{logMAR}$ /year. EZ was measured in eight eyes with mean BL and LFU length of $1287.75 \pm 413.42 \mu \mathrm{m}$ and $686.38 \pm 382.87 \mu \mathrm{m}$ respectively with reduction of $137.01 \mu \mathrm{m} /$ year. Mean BL and LFU CMT were $166.75 \pm 44.39 \mu \mathrm{m}$ and $152.4 \pm 17.05 \mu \mathrm{m}$, respectively, while declining $1.70 \mu \mathrm{m} /$ year. Full-field ERG showed nearly undetectable scotopic responses and reduced photopic responses. Pattern ERG was detectable in four of 10 eyes. Common UWF-FAFs presentation were central macular hyperautofluorescence (hyperAF). Semi-automated Kinetic perimetry (SKP) (III4e and V4e) was undetectable in two eyes, restricted nasally in four eyes, showed a superior wedge defect in two eyes and was normal in the remaining two eyes.

Conclusion RPE is the main site of abnormality in MERTKRP with rod and cone photoreceptors affected secondarily. Early visual loss, moderately rapid EZ line reduction and macular hyperAF were apparent in this cohort. EZ measurement was more reliable than CMT to monitor RP progression. The relatively swift decline in those biomarkers indicating visual function suggests a narrow timespan for intervention.

Conflict of interest: JRG : Novartis (C).

Funding: Partly funded by National Health and Medical Research Council (NHMRC) Grants and Ophthalmic Research Institute of Australia (ORIA).

Award applications: Eberhard Dodt Memorial Award, Marmor Award.

Corresponding author email: john.grigg@sydney.edu.au

\section{P6.5 The age-related full-field ERG features in a series} of patients with KCNV2-retinopathy

Leo, Shaun $M^{1,2}$, Georgiou, Michalis ${ }^{1,2}$, Neveu, Magella $M^{1,2}$, Mahroo, Omar $\mathbf{M}^{1,2}$, Webster, Andrew $\mathbf{R}^{1,2}$, Michaelides, Michel ${ }^{1,2}$, Robson, Anthony $\mathbf{G}^{1,2}$

${ }^{1}$ Moorfields Eye Hospital, London, UK, ${ }^{2}$ Institute of Ophthalmology, University College London, London, UK

Purpose To describe the clinical and age-related full-field ERG features in a series of 45 patients with KCNV2retinopathy.

Methods The clinical and electrophysiological findings in 45 patients (age range 4-59 years) with molecularly-confirmed KCNV2-retinopathy were reviewed. Full-field and pattern electroretinography (ERG; PERG) were performed using gold foil electrodes and incorporated the ISCEV standards, except in four young children who underwent ERG testing with skin electrodes using modified protocols. Photopic On-Off ERGs were additionally recorded (stimulus duration $200 \mathrm{~ms}$ ) in the adults. The patient data were compared with ERGs from a control group of healthy subjects (age range 10-79 years; $\mathrm{n}=141$ ). The main full-field ERG components were quantified and compared with age with age-matched control recordings.

Results Marked reduction in visual acuity was documented in all patients within the first decade of life. Common symptoms included photophobia and a large minority complained of nyctalopia and/or nystagmus. There was evidence from retinal imaging to suggest worsening maculopathy with age. Full-field ERGs showed pathognomonic waveform characteristics, but quantitative assessment of corneal recordings revealed a wide range of ERG amplitudes and peak times. Comparison with the control group showed mean peak time (delay) for the DA 0.01ERG, DA 10 ERG a- and b-waves of $61 \mathrm{~ms}, 12 \mathrm{~ms}$ and $6 \mathrm{~ms}$, respectively, and the mean delay in LA30 Hz ERG peak time was $9 \mathrm{~ms}$. The mean amplitude reductions for the DA 0.01 ERG, DA 10 ERG a-waves, LA30 Hz and LA3 ERG aand b-waves were $55 \%, 21 \%, 48 \%, 47 \%$ and $74 \%$, respectively; the DA10 ERG b-waves showed a mean increase of $18 \%$ compared with the mean for the control group, but there was marked variation (range $-24 \%$ to $+70 \%$ ). The ERGs tended to be larger in younger KCNV2 cases, but there was wide variability and the mean rate of age-related decline was indistinguishable from that seen in the control group. The DA and LA ERG peak times were relatively stable when compared with age. Pattern ERGs were undetectable in all cases.

Conclusion KCNV2-retinopathy is an early-onset retinal dystrophy associated with poor visual acuity and severe macular dysfunction. Full-field ERGs are diagnostic and show a rate of age-associated reduction indistinguishable from controls and consistent with largely stable peripheral retinal dysfunction across almost six decades.

Conflict of interest: None.

Funding: None.

Award applications: Eberhard Dodt Memorial Award.

Corresponding author email: shaun.leo@nhs.net

\section{P6.6 Can PERG be misleading in CSR patients?}

\section{Abdelgawad, Randa Hesham Ali ${ }^{1,2}$, Mahmoud, Rabab Aboelmaaty ${ }^{1,2}$}

${ }^{1}$ Ophthalmology Department, Ain Shams University, Cairo, Egypt; ${ }^{2}$ Alwatany Eye hospital, Electrophysiology of Vision Laboratory, Cairo, Egypt

Purpose To illustrate the importance of mfERG in conjunction with PERG in diagnosing macular diseases such as central serous retinopathy (CSR). As CSR is a macular disease, we expect an abnormal PERG. In our study we spotlight that PERG can remain normal in CSR patients with only the mfERG abnormal, detecting the macular dysfunction.

Methods In our observational study, we had five patients, with age ranging from 35 to 50 years, complaining of defective vision. All patients underwent full ophthalmological examination including best corrected visual acuity, IOP measurement by air puff tonometer, fundus examination with indirect 
ophthalmoscopy, OCT and OCT angiography, PVEP, PERG and mfERG.

Results Patients showed well formed P50 waves with normal latencies and normal N35-P50 amplitudes of the PERG; while mfERG showed depressed P1 amplitudes in the central three rings. CSR was confirmed with OCT and OCT angiography.

Conclusion Not all CSR cases have an abnormal PERG. As the pathology starts in the choroid and the outer retina, function of the inner macular layers may still be preserved, making mfERG important to perform as it reflects outer macular layer function. We are continuing to collect more CSR patients to reinforce our hypothesis.

Conflict of interest: None.

Funding: None.

Award applications: None.

Corresponding author email: randanader@live.com

\section{Poster Session 7: Animal Models}

\section{P7.1 L- and M-cone driven ERG signals in macaques}

Kremers, Jan ${ }^{1}$, Aher, Avinash ${ }^{1}$, Parry, Neil ${ }^{2}$, Patel, Nimesh $^{3}$, Frishman, Laura ${ }^{3}$

${ }^{1}$ Dept. of Ophthalmology, University Hospital Erlangen, Erlangen, Germany; ${ }^{2}$ Vision Science Centre, Manchester Royal Eye Hospital, Manchester, UK; ${ }^{3}$ College of Optometry, University of Houston, Houston, TX USA

Purpose To study L- and M-cone driven ERGs of macaques for comparison with those obtained in human subjects.

Methods ERGs were recorded from the right eye of five anesthetized macaques (Macaca mulatta; three males, two females). The 4-colour ganzfeld stimuli were luminance (22\% contrast), L-cone excitation and M-cone excitation (both $22 \%$ cone contrast). Cone isolating stimuli were generated using triple silent substitution. The stimuli were sinusoidal (14 different temporal frequencies; $4-56 \mathrm{~Hz}$ in $4 \mathrm{~Hz}$ steps), $4 \mathrm{~Hz}$ sawtooth and $2 \mathrm{~Hz}$ square wave temporal modulations.

Results The responses to sine-wave stimuli were dominated by the first (fundamental) component but there were more consistent second harmonic components than in humans. The first harmonic components showed multiple maxima and minima. The responses to low frequency luminance stimuli were smaller than those to cone isolating stimuli, where the average ERG L:M ratio was about unity and the L-M phase difference was $180^{\circ}$. At frequencies above $30 \mathrm{~Hz}$, the luminance responses were larger than those to cone isolating stimuli. The averaged L/M ratio was about 2, with large interindividual variability (between 1.5 and 16). The L- and M-cone driven responses had similar phases. The responses to sawtooth and square waves to luminance and cone isolating stimuli displayed substantially different on- and off-responses. The L-on and M-off responses resembled each other as did the L-off and M-on.

Conclusion The responses measured in macaques contained more substantial second harmonic components than those measured in human subjects, suggesting that macaque responses have stronger inner retinal origins (Hood et al., Invest Ophthalmol Vis Sci 2002;43:1673-1685). Similar to the
ERGs in humans, the responses reflect L-M cone opponency at low temporal frequencies and luminance activity above $30 \mathrm{~Hz}$. Luminance signals display an average L:M ratio of 2 indicating that, similar to in humans, they are L-cone dominated. On- and off-responses to cone isolating stimuli reflect mixed L/M cone opponent and luminance features in both macaques and humans.

Conflict of interest: None.

Funding: German Research Council (DFG) KR1317/13-2. Award applications: None.

Corresponding author email: jan.kremers@uk-erlangen.de

P7.2 The effects of postnatal hyperoxia on the aging Sprague-Dawley rat retina-a study of retinal structure and function

Dorfman, Allison ${ }^{1,2}$, Chaychi, Samaneh ${ }^{1}$, Polosa, Anna ${ }^{1}$, Chemtob, Sylvain ${ }^{1,2,3}$, Lachapelle, Pierre ${ }^{1}$

${ }^{1}$ Department of Ophthalmology/Neurology-Neurosurgery, McGill University-Montreal Children's Hospital Research Institute, Montreal, Quebec, Canada; ${ }^{2}$ Department of Ophthalmology, Centre Hospitalier Universitaire SainteJustine Research Center, Montreal, Quebec, Canada; ${ }^{3}$ Pediatrics and Pharmacology, Centre Hospitalier Universitaire Sainte-Justine Research Center, Montreal, Quebec, Canada

Purpose Postnatal hyperoxia in albino Sprague-Dawley (SD) rats triggers severe and irreversible impairment of retinal structure and function early in life. We aimed to investigate whether these consequences of hyperoxia progressed over time. Methods SD rats were exposed to $80 \% \mathrm{O}_{2}$ from birth to age 14 days $(\mathrm{P} 0-14 ; \mathrm{n}=12)$. Scotopic and photopic ERGs were recorded at $\mathrm{P} 30, \mathrm{P} 60, \mathrm{P} 100, \mathrm{P} 200$ and $\mathrm{P} 300$ and retinal histology was obtained from P15 to P300.

Results In normoxic rats, we observed a rapid decline in ERG b-wave amplitudes between P30 and P60 which was followed by a more subtle decay up to P300 $(p>0.05)$. In contrast, in hyperoxic rats, the rod Vmax and photopic b-wave were nearly maximally attenuated at P30 and reached less than $40 \%$ of P30 values at P300 compared to $32 \%$ for the scotopic b-wave. The a-wave was significantly less affected in the early phase (P30 P60) but rapidly declined afterwards to reach $19 \%$ of P30 values at P300. Hyperoxic retinas disclosed a thinner OPL at $\mathrm{P} 17$ in the central part of the superior retina and at P24 in the central part of the inferior retina. Between P60-P300 the posterior pole became devoid of an OPL with only a peripheral ring of intact OPL remaining. Of interest, with time, the hyperoxic retinopathy also involved the outer retina, and by P300, a similar zone of central damage affecting the photoreceptor layer was also observed with relative sparing of the peripheral retina.

Conclusion Until P60, the functional consequences of oxygeninduced retinopathy (OIR) are largely limited to attenuation of the scotopic and photopic b-waves, whereas the a-wave is preserved to a significantly greater extent. Evaluation at later time points (namely P100, 200 and 300) culminates in photoreceptor dysfunction, whereby the a-wave becomes progressively attenuated. Our findings suggest that the 
pathogenesis of OIR begins initially as a post-receptoral disorder (mainly OPL) which is permanent, whereas the chronic phase is also characterized by damage to the photoreceptor structure and function. It remains to be determined if limiting damage to the inner retina could prevent the long-term chronic structural and functional damage to the photoreceptors that we observed.

Conflict of interest: None.

Funding: CIHR.

Award applications: None.

Corresponding author email: allison.dorfman@mcgill.ca

\section{P7.3 Electrophysiological abnormalities in the 5xFAD mouse model of Alzheimer's disease: Comparison to normal aging}

McAnany, J Jason', Matei, Nathanael' ${ }^{2}$, Liu, Karen', Park, Jason' ${ }^{1}$, Shahidi, Mahnaz ${ }^{2}$

${ }^{1}$ Department of Ophthalmology and Visual Sciences, University of Illinois at Chicago, Chicago, USA;

${ }^{2}$ Department of Ophthalmology, University of Southern California, Los Angeles, California, USA

Purpose Common characteristics of Alzheimer's disease (AD) are observed in 5xFAD transgenic mice at a relatively young age. Retinal dysfunction has been studied in young 5xFAD mice using the electroretinogram (ERG), which has shown amplitude losses and timing delays similar to those observed in older wild type (WT) mice. The purpose of this study was to compare the full-field ERG of young $5 \mathrm{xFAD}$ mice to agesimilar WT mice and to older WT mice to determine the extent to which $5 x F A D$ mutations mimic normal aging.

Methods Dark-adapted (DA) and light-adapted (LA) flash ERGs were recorded from $225 \mathrm{xFAD}$ mice at 3 months of age, $13 \mathrm{WT}$ mice at 3 months of age (WT3), and $15 \mathrm{WT}$ mice at 9 months of age (WT9). ERGs were recorded in response to achromatic flashes of 0.01 to $25 \mathrm{~cd} \mathrm{~s} / \mathrm{m}^{2}$. Following $2 \mathrm{~h}$ of dark adaptation, scotopic responses were measured in the dark. Photopic responses were measured against a $30 \mathrm{~cd} / \mathrm{m}^{2}$ adapting field after $10 \mathrm{~min}$ of light adaptation. Amplitude and implicit time (IT) of the a- and b-waves were calculated according to convention. Oscillatory potentials (OPs) were extracted by bandpass filtering; the amplitude and IT of the OPs were summed to provide a single measure of OP amplitude and IT. In addition, the amplitude and IT of the photopic negative response $(\mathrm{PhNR})$ was measured from the light-adapted recordings. Two-way repeated measures analysis of variance and Holm-Sidak pairwise comparisons were used to compare the amplitudes and ITs of the 5xFAD mice and the two WT groups.

Results There were significant differences between the WT3 and WT9 groups in outer- and inner-retina function under DA conditions: a-wave, b-wave, and OP amplitude differed (all $\mathrm{t}>2.98, p<0.02$ ), but IT did not (all $\mathrm{t}<1.31, p>0.19$ ). DA outer-retinal function did not differ between the 5xFAD and WT3 groups (a-wave amplitude: $\mathrm{t}=1.48, p=0.15$ ), but innerretinal responses differed (b-wave amplitude: $t=3.56$, $p=0.002$; OP IT: $\mathrm{t}=3.71, p=0.001)$. Similar patterns were observed under LA conditions: the WT3 and WT9 a-wave, b-wave, and PhNR amplitudes differed (all $\mathrm{t}>3.34$, $p<0.006$ ); there were no significant IT differences (all $\mathrm{t}<2.24, p>0.05$ ). LA outer-retina function did not differ between the 5xFAD and WT3 groups (a-wave amplitude: $\mathrm{t}=1.87, p=0.13$ ), but inner-retina responses differed (OP amplitude: $\mathrm{t}=3.56, p=0.002 ; \mathrm{PhNR}$ amplitude $\mathrm{t}=5.50$, $p<0.001)$.

Conclusion Age and AD affect the ERG, but the pattern of abnormality is not the same. Older WT mice have reduced photoreceptor function, as evidenced by a-wave amplitude reduction, which affects the downstream bipolar and retinal ganglion cell responses. In contrast, young $5 x F A D$ mice have retinal abnormalities that appear to be selective for the middle/ inner retina. Thus, the 5xFAD mouse model does not represent 'premature aging' of the neural retina but rather specific effects of $\mathrm{AD}$ on post-receptor function.

Conflict of interest: None.

Funding: NIH grants EY017918, EY029220, EY001792 and the Research to Prevent Blindness Foundation.

Award applications: Marmor Award.

Corresponding author email: jmcana1@uic.edu

P7.4 Analysis of rodent scotopic oscillatory potentials at extremely dim flashes shown in time and frequency domains

Polosa, Anna ${ }^{1 *}$, Gauthier, Mercedes ${ }^{1 *}$, Lachapelle, Pierre $^{1}$

*Co-first authors

Department of Ophthalmology, Research Institute of the McGill University Hospital Centre, Montreal, Québec, Canada

Purpose To compare dim scotopic oscillatory potentials (OPs) in small rodents using two different methods: time domain and frequency domain.

Methods Scotopic (strength: -6.3 to $0.9 \log \mathrm{cd} \mathrm{s} / \mathrm{m}^{2} ; 12 \mathrm{~h}$ of dark-adaptation (DA)) ERGs and OPs were obtained from adult albino and pigmented rats and mice. Time-domain analysis included duration of the OP burst and the number of OPs included in the burst. Frequency-domain analysis included fast Fourier transform (FFT) and continuous wavelet transform (CWT).

Results Compared to rat, mouse scotopic OPs had a lower burst threshold (starting at $-6.3 \log \mathrm{cd} \mathrm{s} / \mathrm{m}^{2}$; lowest intensity available), were more numerous (only at dim intensities) and had longer duration. In pigmented strains only, a distinction between an earlier (30-40 ms versus $30-80 \mathrm{~ms}$, rats versus mice, respectively) and faster $(\sim 110 \mathrm{~Hz})$ versus a later (50-100 ms versus $90-150 \mathrm{~ms}$, rats versus mice, respectively) and slower $(\sim 75 \mathrm{~Hz})$ OP burst was evidenced only at dim flashes $\left(<2.7 \log \mathrm{cd} \mathrm{s} / \mathrm{m}^{2}\right.$, more notable in mice). In contrast, this OP burst split was less defined in albino strains (i.e. accordion-shaped like OP burst). In addition, frequencydomain analysis revealed a horizontal [i.e. longer duration $(\sim 100 \mathrm{~ms})]$ frequency spike in mice (versus $\sim 40 \mathrm{~ms}$ in rats), while rats presented a more vertical (i.e. higher frequency 
range $(60-250 \mathrm{~Hz}$, versus $60-140 \mathrm{~Hz}$ in mice) one. With higher stimulus strength $\left(>-2.7 \log \mathrm{cd} \mathrm{s} / \mathrm{m}^{2}\right)$, the morphology and the number of OPs ( \pm 6 OPs) was similar between all groups.

Conclusion Taken together, at dim flashes, our results strongly suggest significant physiological differences in mouse and rat scotopic OP generators. We have identified two OP bursts at very dim flashes that could provide key information in identifying the exact intra-retinal origin of the OPs. Furthermore, our data show that despite very dim flashes, OPs are still present in the signal.

Conflict of interest: None.

Funding: CIHR.

Award applications: Eberhard Dodt Memorial Award.

Corresponding author email: anna.polosa@mail.mcgill.ca

P7.5 The long-flash offset d-wave may reflect ganglion cell function in the chicken retina

\section{Afari, Clement ${ }^{1}$, McCulloch, Daphne $\mathrm{L}^{1}$, Choh, Vivian ${ }^{1}$ \\ School of Optometry and Vision Science, University of Waterloo, Waterloo, Canada}

Purpose We investigated the contribution of ganglion cells to the on- and off-responses in the long-flash ERG in chickens. Methods Full-field long-flash ERGs were recorded bilaterally in anesthetized (ketamine/xylazine) White Leghorn (Gallus gallus domesticus) hatchlings (day 1) with dilated pupils, and again at days 3,14 and 21. The chicks were divided into two groups of five birds each. One group received uniocular surgical optic nerve section (ONS) immediately following the collection of the ERG on day 1 (ONS group). The other group received uniocular intraocular tetrodotoxin (TTX) injections one hour prior to recording ERGs subsequent to day 1 (TTX group). Fellow eyes had sham surgery (ONS group) or were injected with inert vehicle (TTX group). Stimuli were $250 \mathrm{~cd} \mathrm{~s} / \mathrm{m}^{2}$ red flashes on a $30 \mathrm{~cd} / \mathrm{m}^{2}$ blue background (narrow-band LEDs, peak $\lambda 650$ and 462, respectively), the colour combination producing the clearest signals in pilot studies. We recorded amplitudes and implicit times of onset aand $b$-waves and of offset d-waves.

Results Onset and offset ERG waveforms in the chick were similar to those of humans and other species. For both ONS and TTX treatment groups, the offset d-wave was depressed in the treated eyes across the age range (ONS: $p=0.029$; TTX: $p=0.034$ ) with no difference in implicit time (ONS: $p=0.104$; TTX: $p=0.117$ ). Onset ERG a- and b-waves did not differ between the eyes.

Conclusion Our data support the contention that, unlike in mammalian species, offset responses in chicken retinae may reflect the function of inner retinal neurons, as has also been observed in amphibians. As in other vertebrates, d-waves in chicks are presumably generated by off-bipolar cells. Our data suggest that spiking cells, particularly in species with a centrifugal visual system, may enhance the function of offbipolar cells.

Conflict of interest: None.
Funding: NSERC Discovery grant.

Award applications: None.

Corresponding author email: clement.afari@uwaterloo.ca

P7.6 Avian electroretinograms (ERGs) lack the 'ganglion cell-associated' photopic negative response (PhNR) and scotopic threshold response (STR) waveforms

Choh, Vivian ${ }^{1}$, Afari, Clement ${ }^{1}$, McCulloch, Daphne $L^{1}$

School of Optometry and Vision Science, University of Waterloo, Waterloo, Canada

Purpose We aimed to develop a chicken model of ganglion cell function and maturation using full-field ERGs.

Methods Full-field ERGs were recorded bilaterally in anaesthetised (ketamine/xylazine) White Leghorn chicks (Gallus gallus domesticus) with dilated pupils, at hatch (day 1, untreated) and then again at 3, 5, 7, 14 and 21 days, one hour after uniocular injection with tetrodotoxin (TTX) to block function of spiking cells of the retina. In five chicks, a darkadapted (DA) stimulus:response series (14 steps from -6.0 to $+1.5 \log \mathrm{cd} \mathrm{s} / \mathrm{m}^{2}$ ) was recorded. A minimum of 20 sweeps were averaged for weak stimuli ( -6.0 to $\left.-2.5 \log \mathrm{cd} \mathrm{s} / \mathrm{m}^{2}\right)$. The mid-range minimum was 10 sweeps and for stronger stimuli $\left(-1.5\right.$ to $\left.+1.5 \log \mathrm{cd} \mathrm{s} / \mathrm{m}^{2}\right)$, four or more sweeps were averaged. A light-adapted series of red flashes (at quarter-log steps from -1 to $0.9 \log \mathrm{cd} \mathrm{s} / \mathrm{m}^{2}$ ) on a blue, rod-suppressing background $\left(30 \mathrm{~cd} / \mathrm{m}^{2}\right)$ was recorded in a separate cohort of six chicks at the same ages and with the same uniocular treatment. In addition to conventional a- and b-wave measures, darkadapted ERGs to weak stimuli were examined for the STR negativity. The PhNR measured to the b-wave in light-adapted ERGs was assessed for saturated amplitude and sensitivity with Naka-Rushton least squares curve fitting.

Results An STR negativity was not detectable in either eye of the chicks for any of the stimulus strengths in the DA ERGs at any time point. Across the age range, the PhNR measured to the b-wave did not fall significantly below the pre-stimulus baseline and TTX did not affect the PhNR-saturated amplitude or sensitivity ( $p=0.106$, and $p=0.166$, respectively). As expected, TTX did not affect the a- or b-waves generated distal to the retinal ganglion cells.

Conclusion Our protocol is similar to that used to measure STRs in other species and was verified to elicit a clear STR in a human. An absent STR in the cone-dominated chicken retina might be attributed to insufficient rod input. However, the absence of a PhNR in chicks with both functioning and blocked ganglion cells was unexpected. We suggest that the intraretinal glia found in species with an inner retinal blood supply may play a role in these ERG waveforms associated with ganglion cell function.

Conflict of interest: None.

Funding: NSERC Discovery Grant.

Award applications: None.

Corresponding author email: vchoh@uwaterloo.ca 